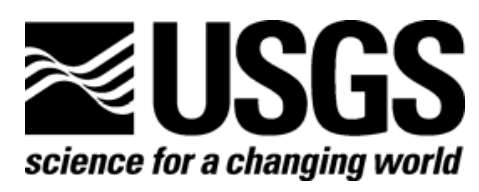

\title{
Geosoft eXecutables (GX's) Developed by the U.S. Geological Survey, Version 2.0, with Notes on GX Development from Fortran Code
}

By Jeffrey D. Phillips

Open-File Report 2007-1355

U.S. Department of the Interior

U.S. Geological Survey 


\title{
U.S. Department of the Interior DIRK KEMPTHORNE, Secretary
}

\author{
U.S. Geological Survey \\ Mark D. Myers, Director
}

U.S. Geological Survey, Reston, Virginia 2007

For product and ordering information:

World Wide Web: http://www.usgs.gov/pubprod

Telephone: 1-888-ASK-USGS

For more information on the USGS - the Federal source for science about the Earth, its natural and living resources, natural hazards, and the environment:

World Wide Web: http://www.usgs.gov

Telephone: 1-888-ASK-USGS

Any use of trade, product, or firm names is for descriptive purposes only and does not imply endorsement by the U.S. Government.

Although this report is in the public domain, permission must be secured from the individual copyright owners to reproduce any copyrighted material contained within this report.

Suggested citation:

Phillips, J.D., 2007, Geosoft eXecutables (GX's) developed by the U.S. Geological Survey, version 2.0, with notes on GX development from Fortran code: U.S. Geological Survey Open-File Report 2007-1355. 
Contents

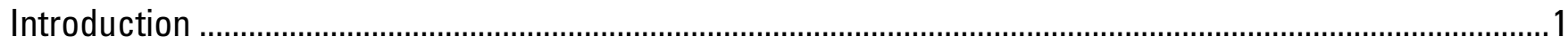

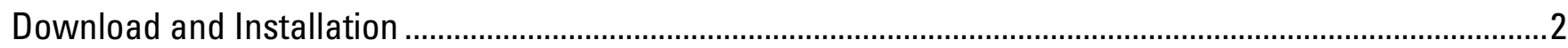

The USGS Menu Files and Short GX Descriptions .....................................................................................

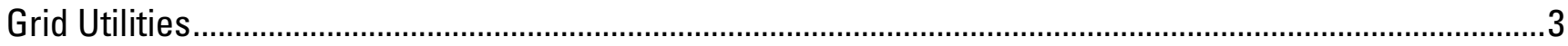

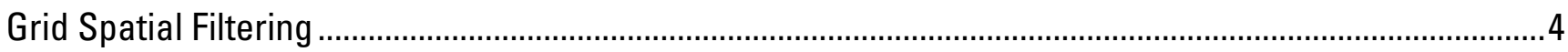

Grid Fourier Filtering - Step-by-Step .......................................................................................................

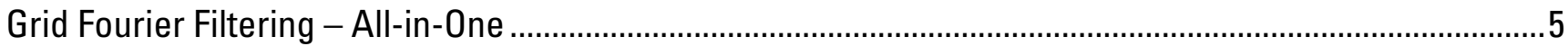

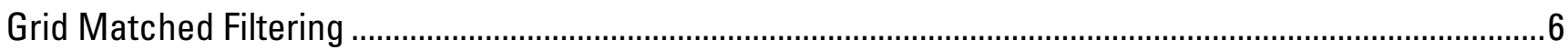

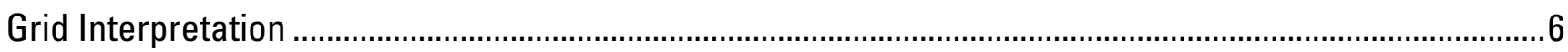

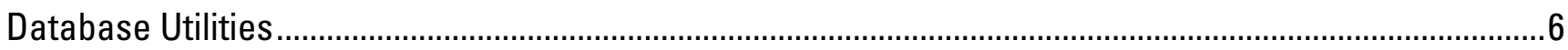

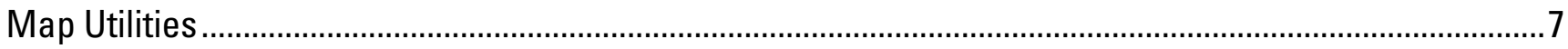

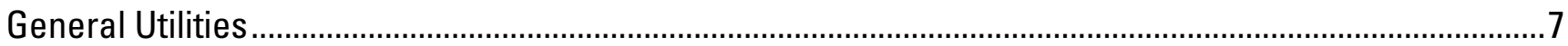

Temporary and Ancillary Files Generated and Used by the GX's...............................................................

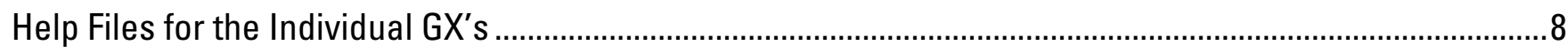

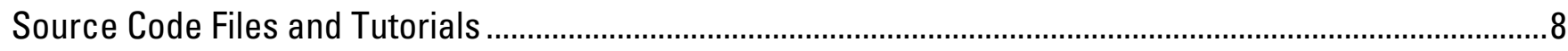

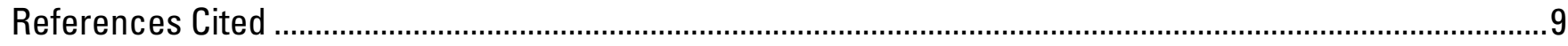

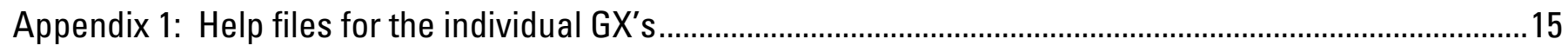

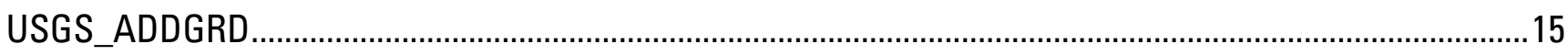

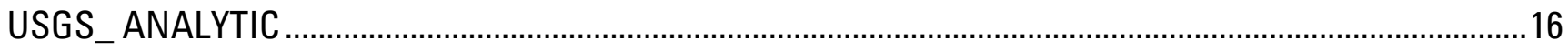

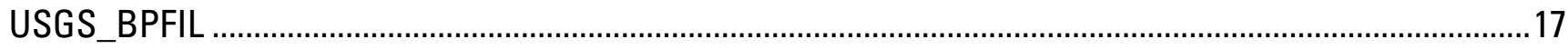

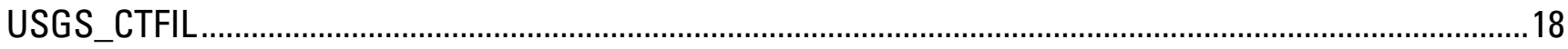

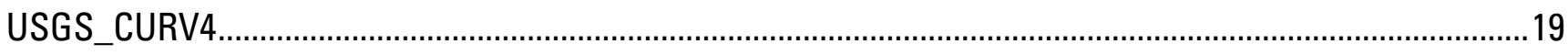




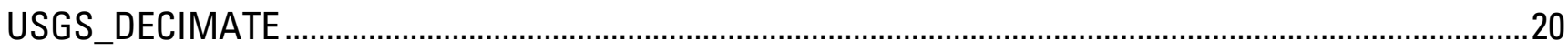

USGS_DECOR

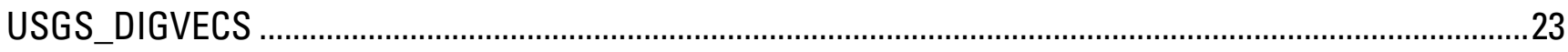

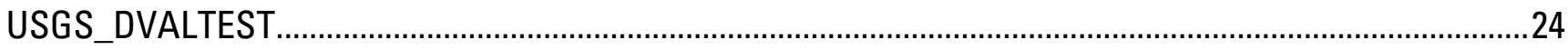

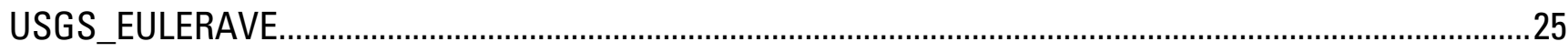

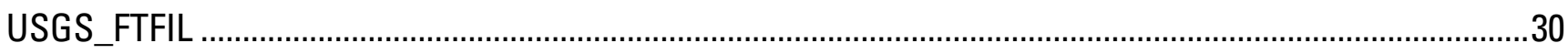

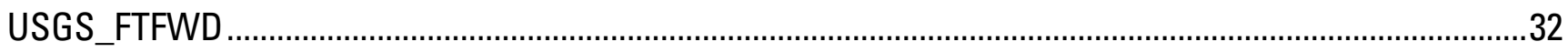

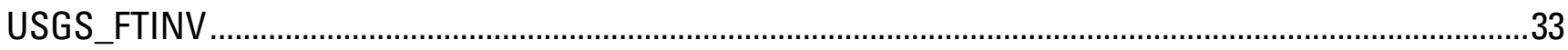

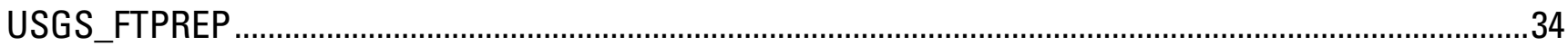

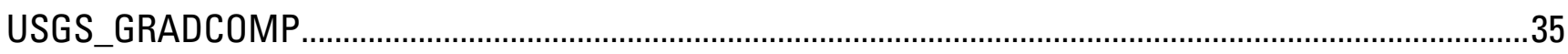

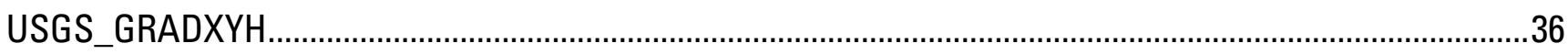

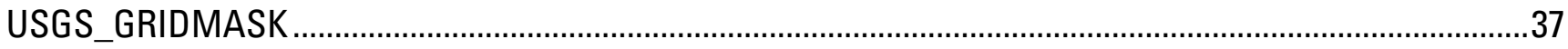

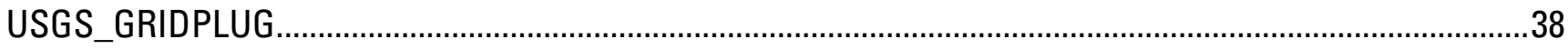

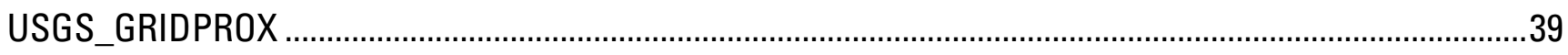

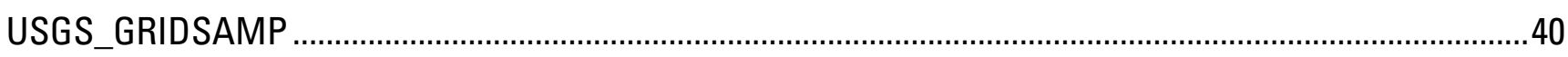

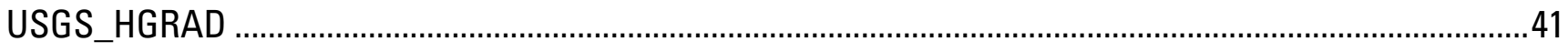

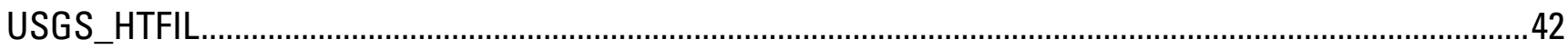

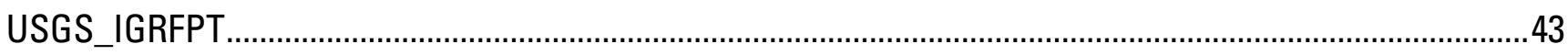

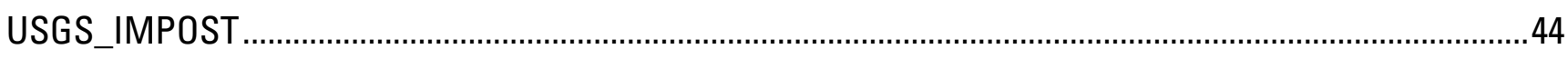

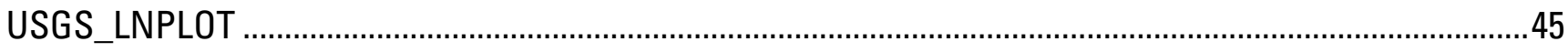

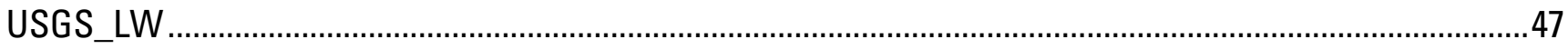

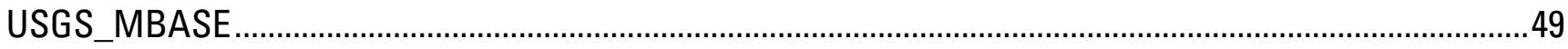

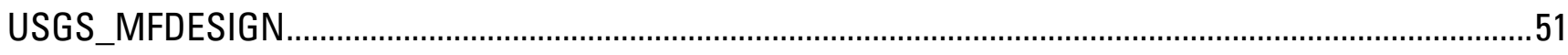




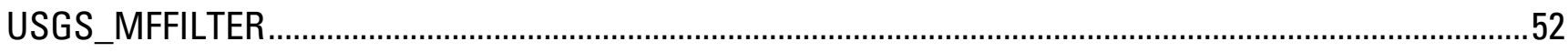

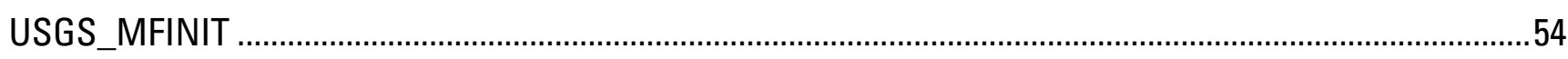

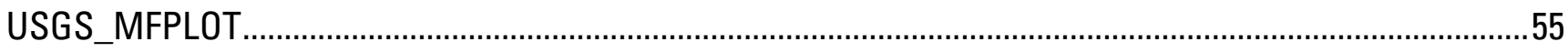

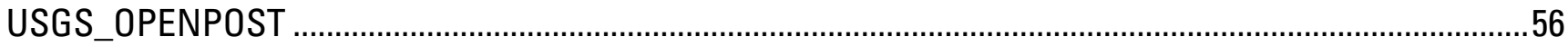

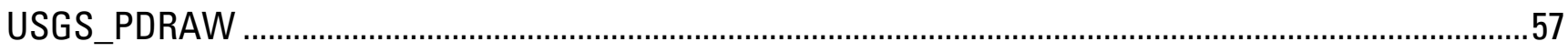

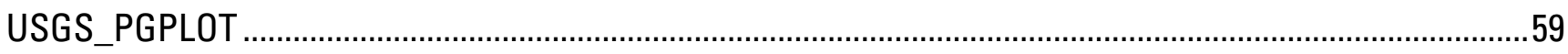

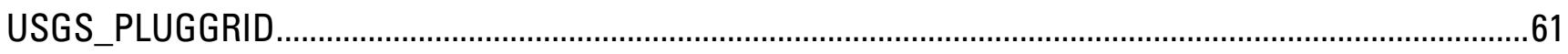

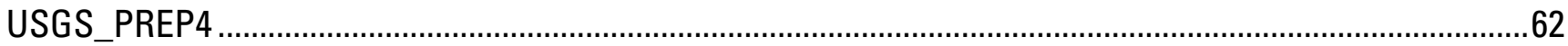

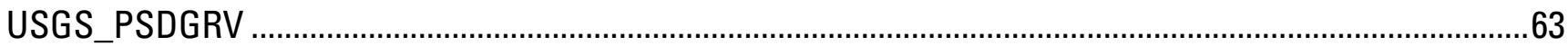

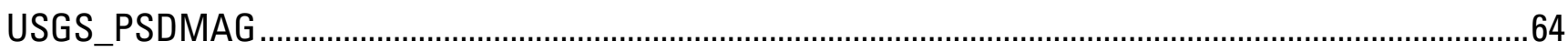

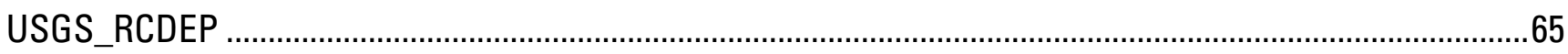

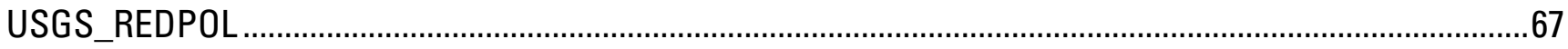

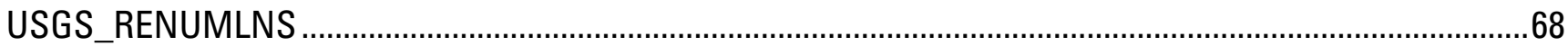

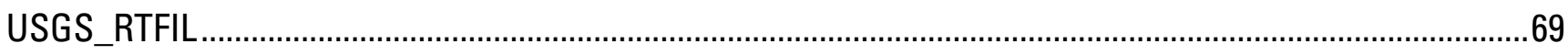

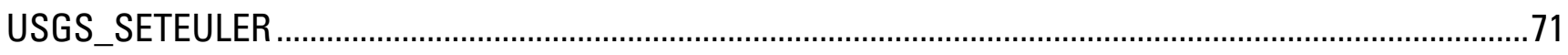

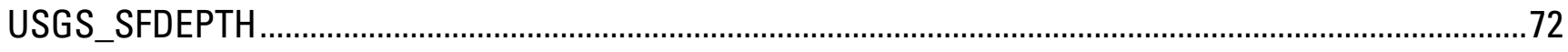

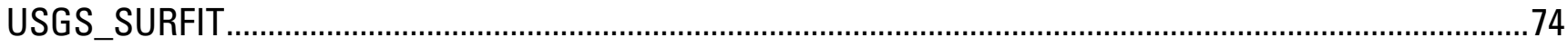

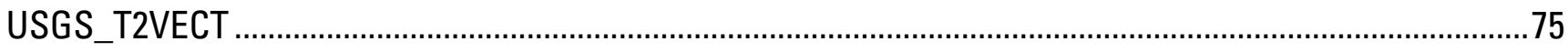

USGS_TGRAD

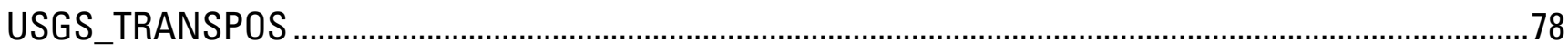

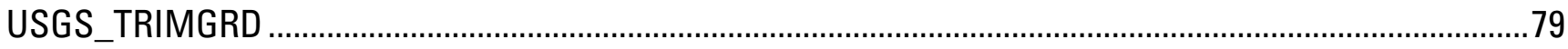

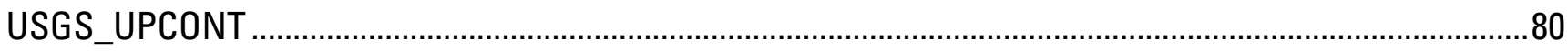

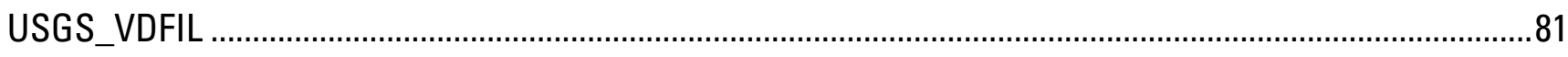


USGS_VERTINT. .82

Appendix 2: Converting FORTRAN code to a Geosoft GX using the GNU g77 and gcc compilers .83

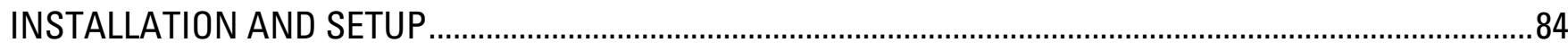

GETTING STARTED

STEP 0: CREATING THE LIBRARIES AND FORTRAN WRAPPER FUNCTIONS ……….................................85

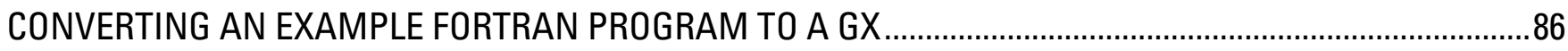

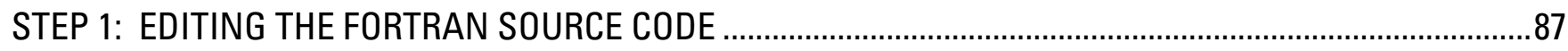

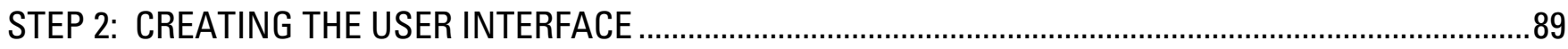

STEP 3: CREATING THE GX SOURCE CODE

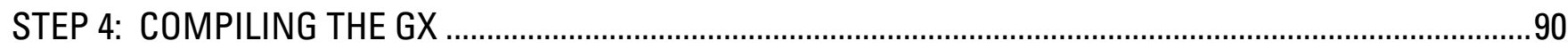

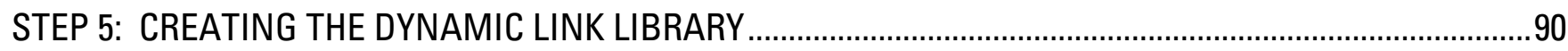

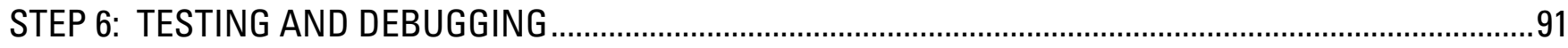

\section{Tables}

Table 1. Listing of usgs.omn ..........................................................................................................11

Table 2. Listing of usgsv.omn ...................................................................................................

Table A1. Listing of Makefile for creating the Geosoft libraries.

Table A2. Part 1 of the edited Fortran source code file, usgs_curv4.f..........................................................95

Table A3. Part 2 of the edited Fortran source code file..................................................................................96

Table A4. Part 3 of the edited Fortran source code file...............................................................................97

Table A5. Part 4 of the edited Fortran source code file................................................................................98

Table A6. Part 5 of the edited Fortran source code file.................................................................................99

Table A7. Part 6 of the edited Fortran source code file..............................................................................102

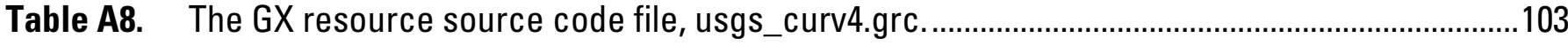




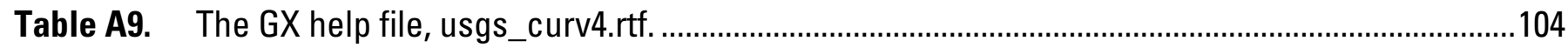

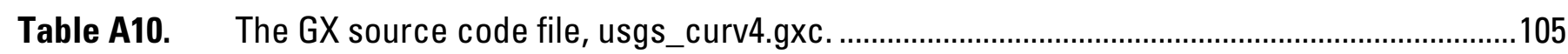

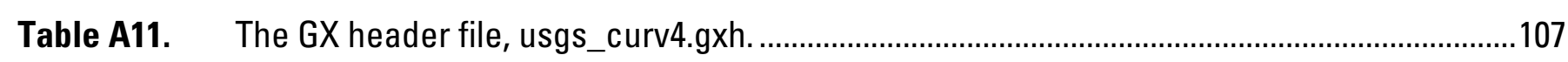

Table A12. Code fragment added to the end of the wrapper functions header file, wrappers.h.............108

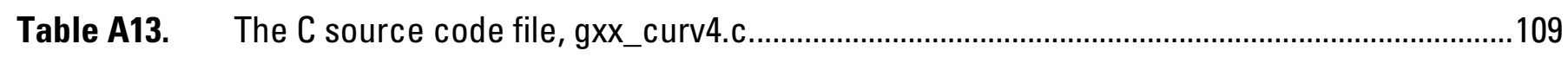

Table A14. The makefile for creating the GX dynamic-link library, Makefile..........................................110 


\section{Geosoft eXecutables (GX's) Developed by the U.S. Geological Survey, Version 2.0, with Notes on GX Development from Fortran Code}

By Jeffrey D. Phillips

\section{Introduction}

Geosoft executables (GX's) are custom software modules for use with the Geosoft Oasis montaj $^{\mathrm{TM}}$ geophysical data processing system, which currently runs under the Microsoft Windows ${ }^{\mathrm{TM}}$ 2000 or XP operating systems. The U.S. Geological Survey (USGS) uses Oasis montaj ${ }^{\mathrm{TM}}$ primarily for the processing and display of airborne geophysical data. The ability to add custom software modules to the Oasis montaj ${ }^{\mathrm{TM}}$ system is a feature employed by the USGS in order to take advantage of the large number of geophysical algorithms developed by the USGS during the past half century.

This main part of this report, along with Appendix 1, describes Version 2.0 GX's developed by the USGS or specifically for the USGS by contractors. These GX's perform both basic and advanced operations. Version 1.0 GX's developed by the USGS were described by Phillips and others (2003), and are included in Version 2.0. Appendix 1 contains the help files for the individual GX's. 
Appendix 2 describes the new method that was used to create the compiled GX files, starting from legacy Fortran source code. Although the new method shares many steps with the approach presented in the Geosoft GX Developer manual, it differs from that approach in that it uses free, opensource Fortran and $\mathrm{C}$ compilers and avoids all Fortran-to-C conversion.

\section{Download and Installation}

The executable GX files, which will only work from within Geosoft Oasis montaj ${ }^{\mathrm{TM}}$, and the source code files used to develop them can be downloaded using a web browser from ftp://ftpext.usgs.gov. The files are in the /pub/cr/co/denver/musette/pub/gx directory. This location is subject to change, so the best way to locate the files is through the following link.

\section{$<$ GO TO FTP SITE $>$}

Each compiled GX consists of a compiled GX file with the suffix .gx. In addition, there is a global file containing error messages called usgs.err and two menu files called usgs.omn and usgsv.omn. The usgs.omn menu file is intended for use with the licensed version of Oasis montaj ${ }^{\mathrm{TM}}$, and the usgsv.omn menu is intended for use with the free Oasis montaj ${ }^{\mathrm{TM}}$ viewer. Many GX's require a dynamically-linked library file with the suffix .dll, and a few require an executable file with the suffix .exe. In the current release of Oasis montaj ${ }^{\mathrm{TM}}$ (version 6.4), compiled GX files are installed in the $\mathbf{g x}$ subdirectory of the IProgram Files\Geosoft|Oasis montaj directory for the licensed version, and in the IProgram Files\GeosoftlOasis montaj Viewer directory for the free viewer; error files are installed in the corresponding ger subdirectory, menu files are installed in the corresponding omn subdirectory, and dynamic-link library and executable files are installed in the corresponding bin subdirectory. 


\section{The USGS Menu Files and Short GX Descriptions}

The GX's described here can be accessed from a USGS menu that can be added to the menu bar in Oasis montaj ${ }^{\mathrm{TM}}$. The menu structure for use with the licensed version of Oasis montaj ${ }^{\mathrm{TM}}$ is defined in a text file called usgs.omn ( $<$ Table $1>)$. The menu structure for use with the unlicensed (free) Oasis montaj Viewer ${ }^{\mathrm{TM}}$ is defined in a text file called usgsv.omn $(<$ Table $2>)$. These files can be easily modified to add additional categories and custom GX's as they are developed. Some of the GX's referenced in the usgs.omn menu file (those with names that do not start with usgs_) are part of the licensed Oasis montaj ${ }^{\mathrm{TM}}$ package; others (with names that do start with usgs_) use Geosoft library routines that are part of the licensed package. All of the GX's referenced in the usgsv.omn menu file work with the free Oasis montaj viewer as of version 6.4. There is no guarantee that these GX's will work with later versions of the viewer.

The following GX's, listed by menu category, are described in this report. GX's that originally appeared in version 1.0 (Phillips and others, 2003) are indicated by “(from version 1.0)". New GX's that did not appear in version 1.0 are indicated by “(new)". GX's that will likely run only in the licensed version of Oasis montaj are so indicated; all others will run in either the licensed version or the unlicensed Viewer version.

\section{Grid Utilities}

- usgs_addgrid.gx - perform arithmetic operations (including masking) using two grids or using a grid and a constant (new).

- usgs_decimate.gx - decimate (or replicate) a grid by removing (or duplicating) nodes (new).

- usgs_dvaltest.gx - test for dummy (no data) values in a grid (from version 1.0).

- usgs_gridmask.gx - restore holes to a grid from a masking grid (from version 1.0).

- usgs_gridplug.gx - plug holes in a grid by using minimum curvature iterations (from version 1.0). 
- usgs_gridprox.gx - create a grid containing distance to the nearest data point (from version 1.0).

- usgs_pluggrid.gx - plug holes in a grid by regridding (from version 1.0; better than gridplug.gx, but requires a license for gridding).

- usgs_prep4.gx - prepare a grid for Geosoft Fourier transform by plugging holes (if any), and extending the rows and columns (from version 1.0; requires a license for gridding).

- usgs_transpos.gx - transpose a grid (from version 1.0).

- usgs_analytic.gx - total gradient (“3D analytic signal”) from three derivative grids (new).

- usgs_trimgrd.gx - trim a grid back to its data area by removing borders of dummy values (new).

\section{Grid Spatial Filtering}

- usgs_decor.gx - decorrugation of east-west or north-south flight-line noise (new).

- usgs_gradcomp.gx - compute filtered horizontal derivative grids and the horizontal gradient magnitude grid using the gradient-component method of Thurston and Brown (1994) (from version 1.0).

- usgs_gradxyh.gx - compute horizontal derivative grids and the horizontal gradient magnitude grid using local quadratic surfaces (new).

- usgs_hgrad.gx - calculate the magnitude of the horizontal gradient of a grid (from version 1.0; requires licensed grid filtering).

- usgs_surfit.gx - fit a polynomial surface to a grid and calculate the residual grid (new).

\section{Grid Fourier Filtering - Step-by-Step}

- usgs_gridplug.gx - plug holes in a grid by using minimum curvature iterations (from version 1.0).

- usgs_ftprep.gx - prepare (extend) a plugged input grid for Fourier transformation (new). 
- usgs_ftfwd.gx - compute the Fourier transform of a (prepared) grid (new).

- usgs_ftfil.gx - basic Fourier domain filtering (new).

- usgs_htfil.gx - Fourier domain Hilbert transform components of a grid (new).

- usgs_rtfil.gx - regularized Fourier domain filtering (new).

- usgs_bpfil.gx - Fourier domain bandpass, highpass, or lowpass filtering (new).

- usgs_ctfil.gx - Fourier domain transformation of any component (total field, X, Y, or Z) to all other components (new).

- usgs_vdfil.gx - Fourier domain vertical derivative from horizontal derivatives (new).

- usgs_ftinv.gx - compute the inverse Fourier transform of a grid (new).

\section{Grid Fourier Filtering - All-in-One}

- usgs_tgrad.gx - one-step total gradient (3D analytic signal) of a potential-field data grid using either the gradient-component method with optional low-pass filtering or local quadratic surfaces (new).

- usgs_lw.gx - one-step local wavenumber of a potential-field data grid using either the gradient-component method with optional low-pass filtering or local quadratic surfaces (new).

- usgs_psdgrv.gx - one-step pseudogravity transformation of a total-field magnetic data grid (new).

- usgs_psdmag.gx - one-step pseudomagnetic transformation of a gravity data grid (new).

- usgs_redpol.gx - one-step simple reduction-to-the-pole transformation of a grid (new).

- usgs_upcont.gx - one-step upward or downward continuation of a potential-field grid (new).

- usgs_t2vect.gx - one-step total-field grid to vector component grids (new).

- usgs_vertint.gx - vertical integral or derivative of a potential-field grid (new) 


\section{Grid Matched Filtering}

- usgs_mfdesign.gx - design matched bandpass filters (new).

- usgs_mffilter.gx - apply matched bandpass filters (new).

- usgs_mfinit.gx - initialize the matched filtering process (new).

- usgs_mfplot.gx - plot the current matched filters (new).

\section{Grid Interpretation}

- usgs_tgrad.gx - one-step total gradient (analytic signal) of a data grid (new).

- usgs_lw.gx - one-step local wavenumber of a potential-field data grid (new).

- usgs_curv4.gx - extract extrema (highs, lows, ridges, troughs) from a grid using curvature (new).

- usgs_seteuler.gx - prepare a grid for extended Euler deconvolution (new).

- usgs_eulerave.gx - magnetic source location using extended Euler deconvolution (new).

- usgs_gridsamp.gx - sample a grid at the locations of a USGS post file (new).

- usgs_rcdep.gx - magnetic source location using profile analysis on grid rows and columns (new).

- usgs_sfdepth.gx - magnetic source location from a special function (total gradient or local wavenumber) grid (new).

\section{Database Utilities}

- usgs_impost.gx - import a USGS post file into a new database (new).

- usgs_openpost.gx - import a USGS post file into a new database (new; faster but requires license).

- usgs_renumlns.gx - renumber selected lines in a database (from version 1.0). 


\section{Map Utilities}

- usgs_digvecs.gx - digitize vectors from a map and place them in an ASCII XYZ file (from version $1.0)$.

- usgs_lnplot.gx - plot lines from an ASCII line file (new).

- usgs_pdraw.gx - draw, redraw, or append polygons from a .ply file to a specified map group (from version 1.0).

- usgs_pgplot.gx - draw a crude page-sized map surround (new).

\section{General Utilities}

- usgs_igrfpt.gx - calculate the International Geomagnetic Reference Field (IGRF) at a specified location (new).

- usgs_mbase.gx - generate diurnal corrections for magnetic anomaly measurements using data from multiple magnetic base stations (new).

\section{Temporary and Ancillary Files Generated and Used by the GX's}

Many of the GX's generate and use temporary or ancillary files. Temporary files are deleted by the GX prior to a successful completion. Temporary grid files are generated in the default Geosoft format, unless a different default grid format has been selected (licensed version only). Ancillary files are not deleted by the GX, but are left for use by other computer programs. Ancillary files include DXF (AutoCad) drawings, Geosoft ASCII XYZ point and line data files, and USGS binary post files. The USGS post files contain point data with $\mathrm{x}$ (east) and $\mathrm{y}$ (north) coordinates, and six z-channels. The post files, along with USGS PC binary grid files, provide an interface between Oasis montaj and the USGS DOS-based potential-field software package (Phillips, 1997). 
The GX's in this report do not access Geosoft's proprietary .gi files, which contain projection information for grid files. As a consequence, grid files produced by these GX's will have corresponding .gi files with unknown projections. This is a limitation of this software.

\section{Help Files for the Individual GX's}

The help files in Appendix 1 are RTF-formatted files that are compiled into each GX. The help file can be displayed in WordPad by pressing the "??" button in the upper right corner of the GX dialog box. Each help file contains several sections, including a general description of the function of the GX, a list of the interactive parameters that can be entered through the dialog box, a list of the batch parameter names that can be used to call the GX from within another GX, and application notes providing additional information.

\section{Source Code Files and Tutorials}

Each GX is built from a number of source code files. These include, at a minimum, an RTF help file with the suffix .rtf, a GX source code file with the suffix .gxc, and a resource file with the suffix .grc. Some GXs use a dynamic link library compiled from Fortran code. In addition to the three source code files described above, these GXs require the Fortran source code file with a suffix of .f, a C code wrapper function for the Fortran subroutine calls, usually called $\mathbf{g x x} \mathbf{x}_{-}^{*} . \mathbf{c}$; the generic wrapper functions wfuncs.c; the $\mathrm{C}$ header file and wrappers.h, and a .gxh prototype file. The interrelationships of these files are described in Appendix 2, which is a tutorial on GX development from Fortran code. 


\section{References Cited}

Barbosa, V.C.F., Silva, J.B.C. and Medeiros, W.E., 1999, Stability analysis and improvement of structural index estimation in Euler deconvolution: Geophysics, v. 64, no. 1, p. 48-60.

Nabighian, M.N., 1984, Toward a three-dimensional automatic interpretation of potential field data via generalized Hilbert transforms: Fundamental relations: Geophysics, v. 49, no. 6, p. 780-786.

Nabighian, M.N., and Hansen, R.O., 2001, Unification of Euler and Werner deconvolution in three dimensions via the generalized Hilbert transform: Geophysics, v. 66, no. 6, p. 1805-1810.

Oppenheim, A.V., and Schafer, R.W., 1975, Digital Signal Processing: Englewood Cliffs, New Jersey, Prentice-Hall, Inc., 585 p.

Phillips, J.D., 1997, Potential-field geophysical software for the PC, version 2.2: U.S. Geological Survey Open-File Report 97-725, 34 p.

Phillips, J.D., 2000, Locating magnetic contacts: a comparison of the horizontal gradient, analytic signal, and local wavenumber methods: Society of Exploration Geophysicists, Expanded Abstracts with Biographies, 2000 Technical Program, v. 1, p. 402-405.

Phillips, J.D., 2001, Designing matched bandpass and azimuthal filters for the separation of potentialfield anomalies by source region and source type: Australian Society of Exploration Geophysicists, 15th Geophysical Conference and Exhibition, Expanded Abstracts CD-ROM, 4 p. 
Phillips, J.D., 2002, Two-step processing for 3D magnetic source locations and structural indices using extended Euler or analytic signal methods: Society of Exploration Geophysicists, 2002 Technical Program Expanded Abstracts, 4 p.

Phillips, J.D., Duval, J.S., and Saltus, R.W., 2003, Geosoft executables (GXs) developed by the U.S. Geological Survey, version 1.0, with a viewgraph tutorial on GX development: U.S. Geological Survey Open-File Report 03-010, 21 p. http://pubs.usgs.gov/of/2003/ofr-03-010/

Phillips, J.D., Hansen, R.O., and Blakely, R.J., 2007, The use of curvature in potential-field interpretation: Exploration Geophysics, v. 38, p. 111-119.

Reid, A.B., Allsop, J.M., Granser, H., Millett, A.J., and Somerton, I.W., 1990, Magnetic interpretation in three dimensions using Euler deconvolution: Geophysics, v. 55, no. 1, p. 80-91.

Thurston, J.B., and Brown, R.J., 1994, Automated source-edge location with a new variable pass-band horizontal-gradient operator: Geophysics, v. 59, no. 4, p. 546-554.

Urquhart, Ted, 1988, Decorrugation of enhanced magnetic field maps: Society of Exploration Geophysicists Fifty-Eighth Annual Meeting, Expanded Abstracts, p. 371-372. 


\section{Table $1 . \quad$ Listing of usgs.omn}

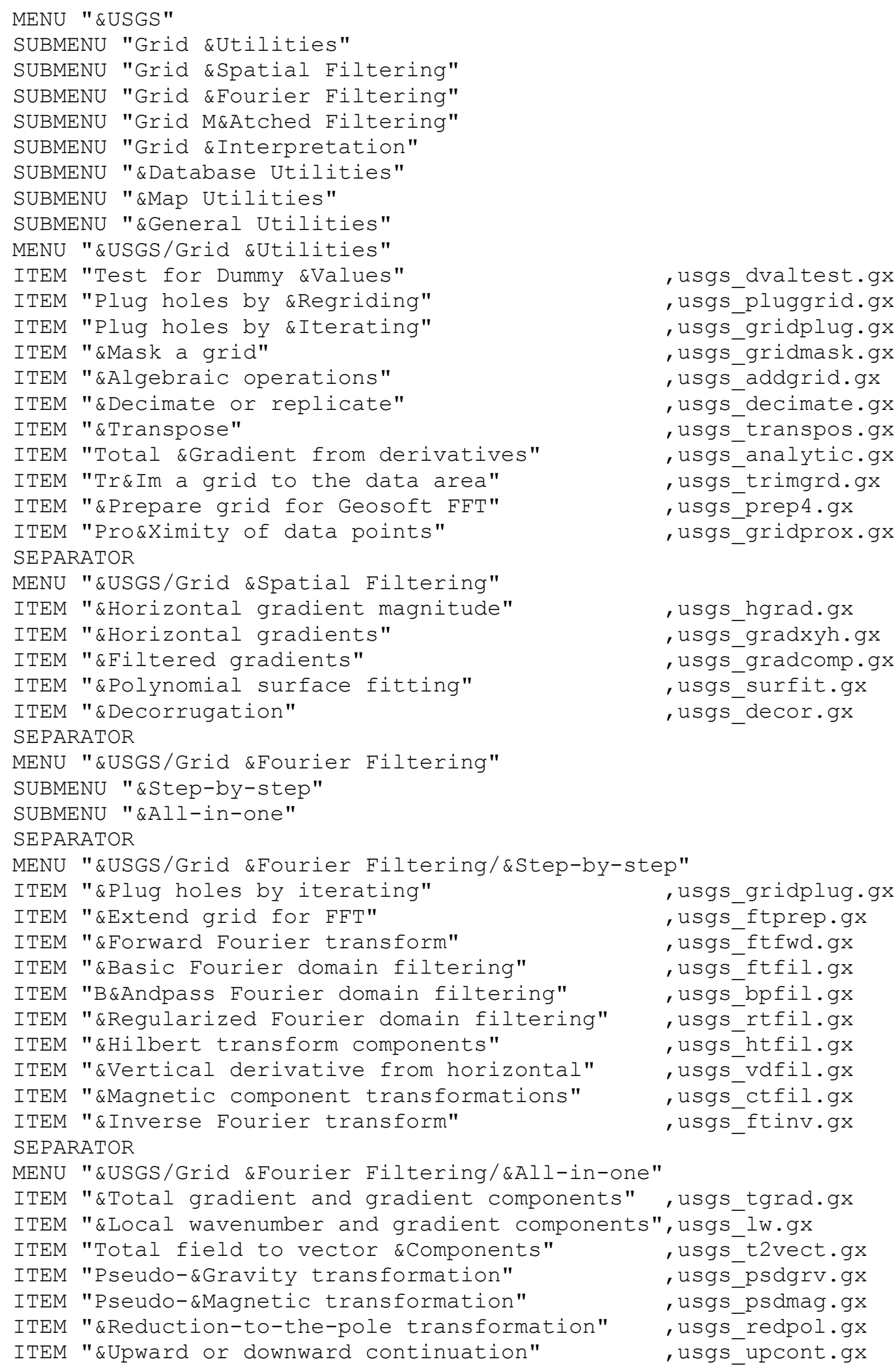




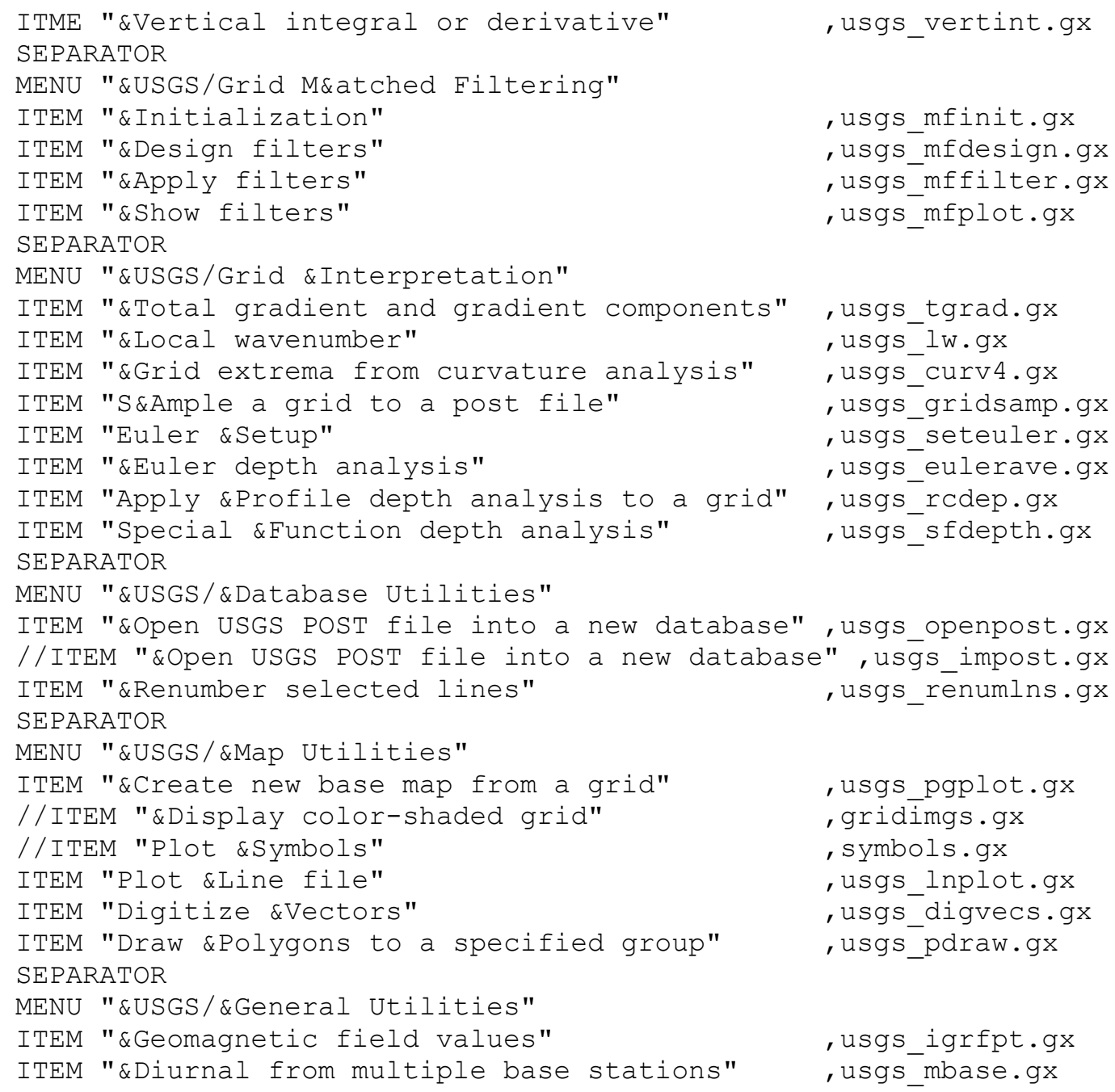




\section{Table 2. Listing of usgsv.omn}

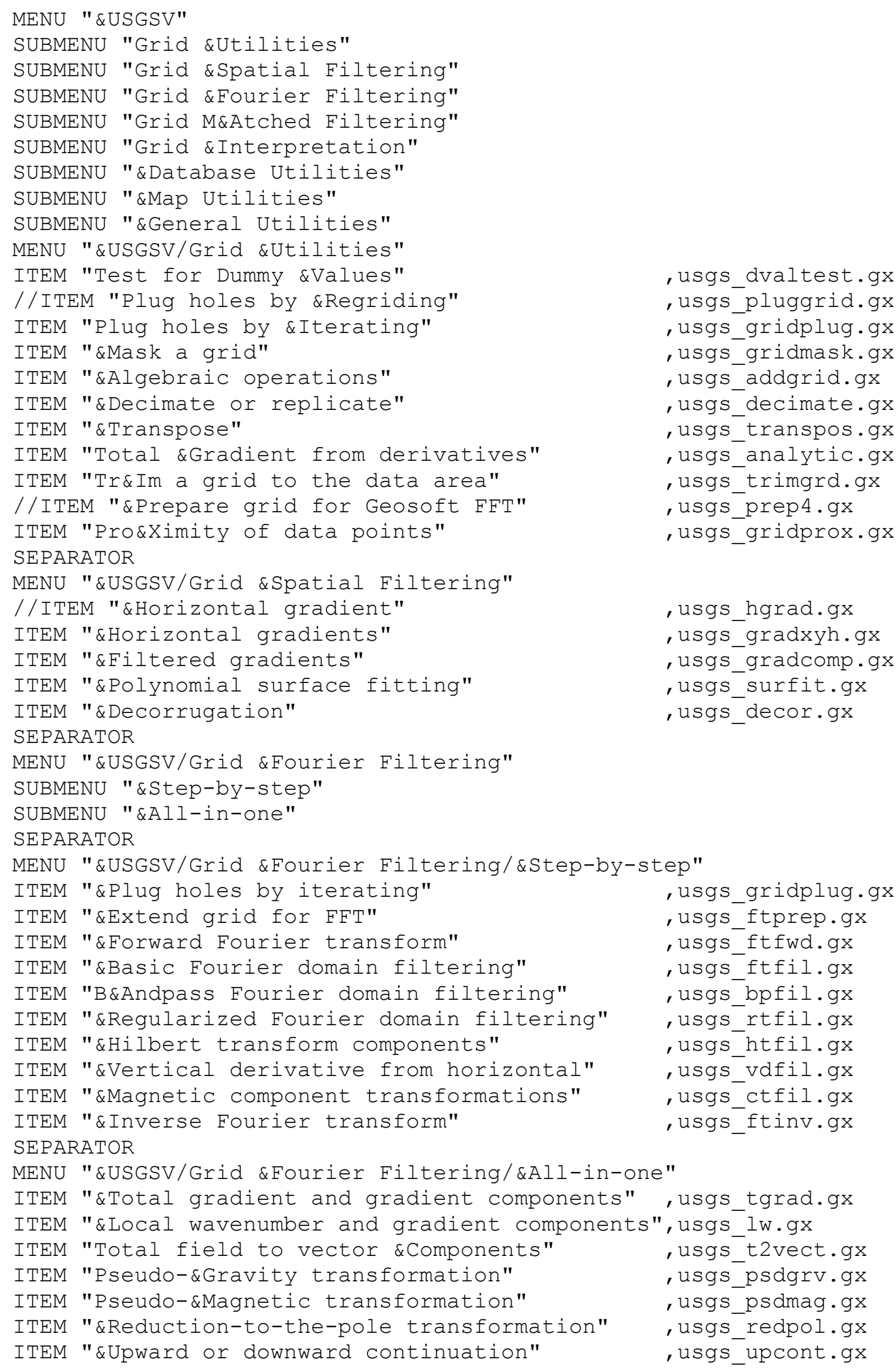




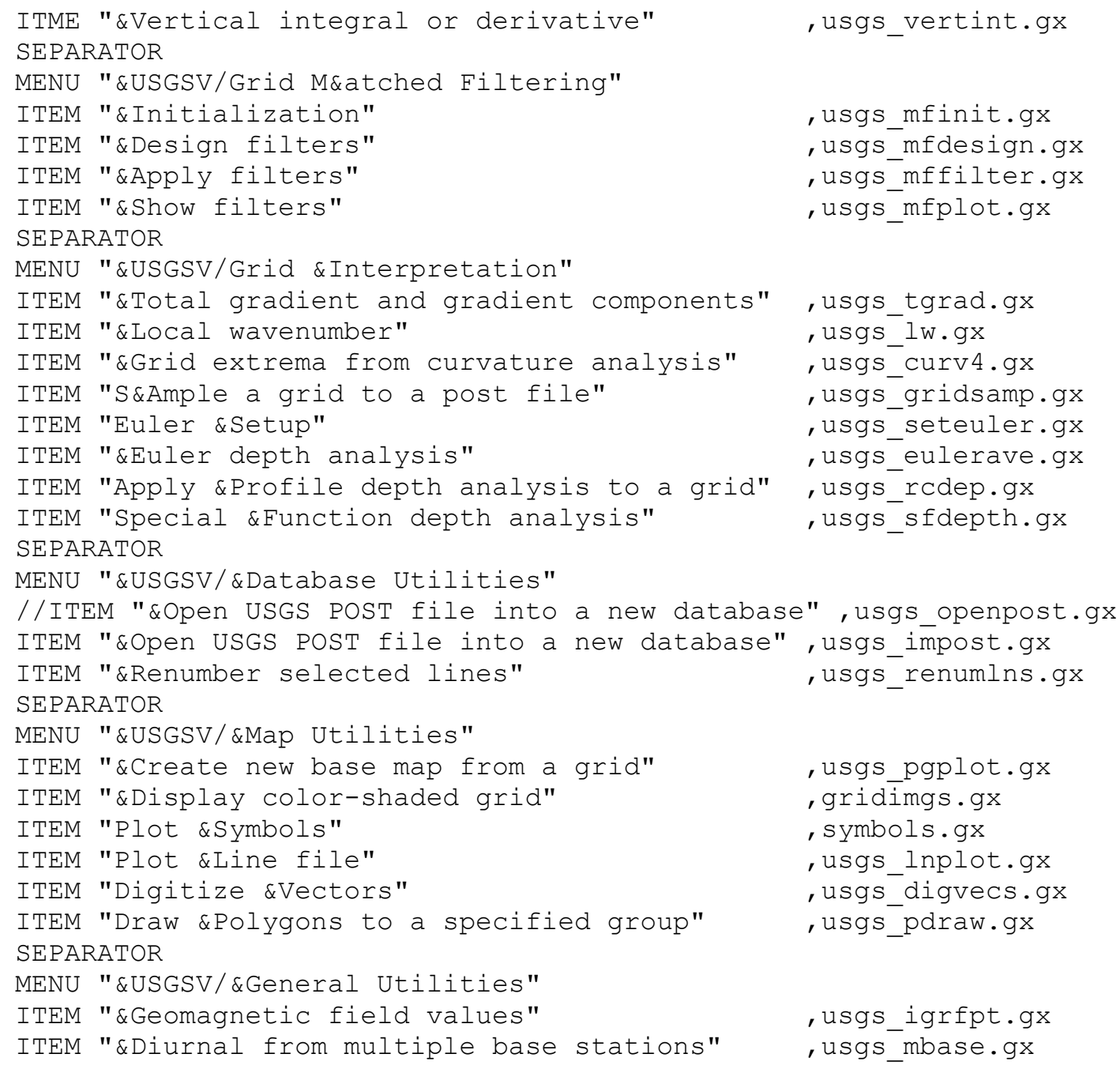




\section{Appendix 1: Help files for the individual GX's}

\section{USGS_ADDGRD}

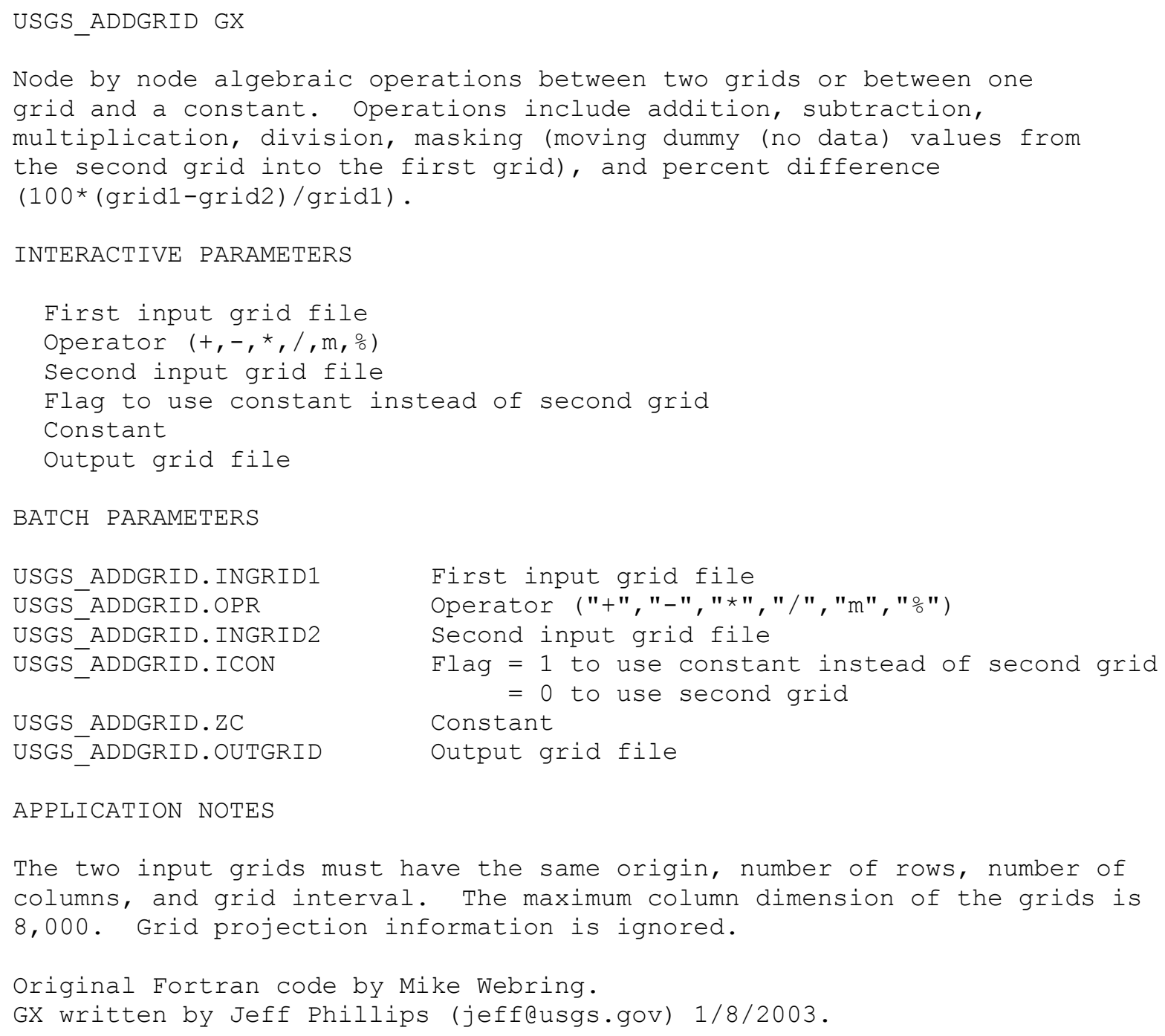




\section{USGS_ANALYTIC}

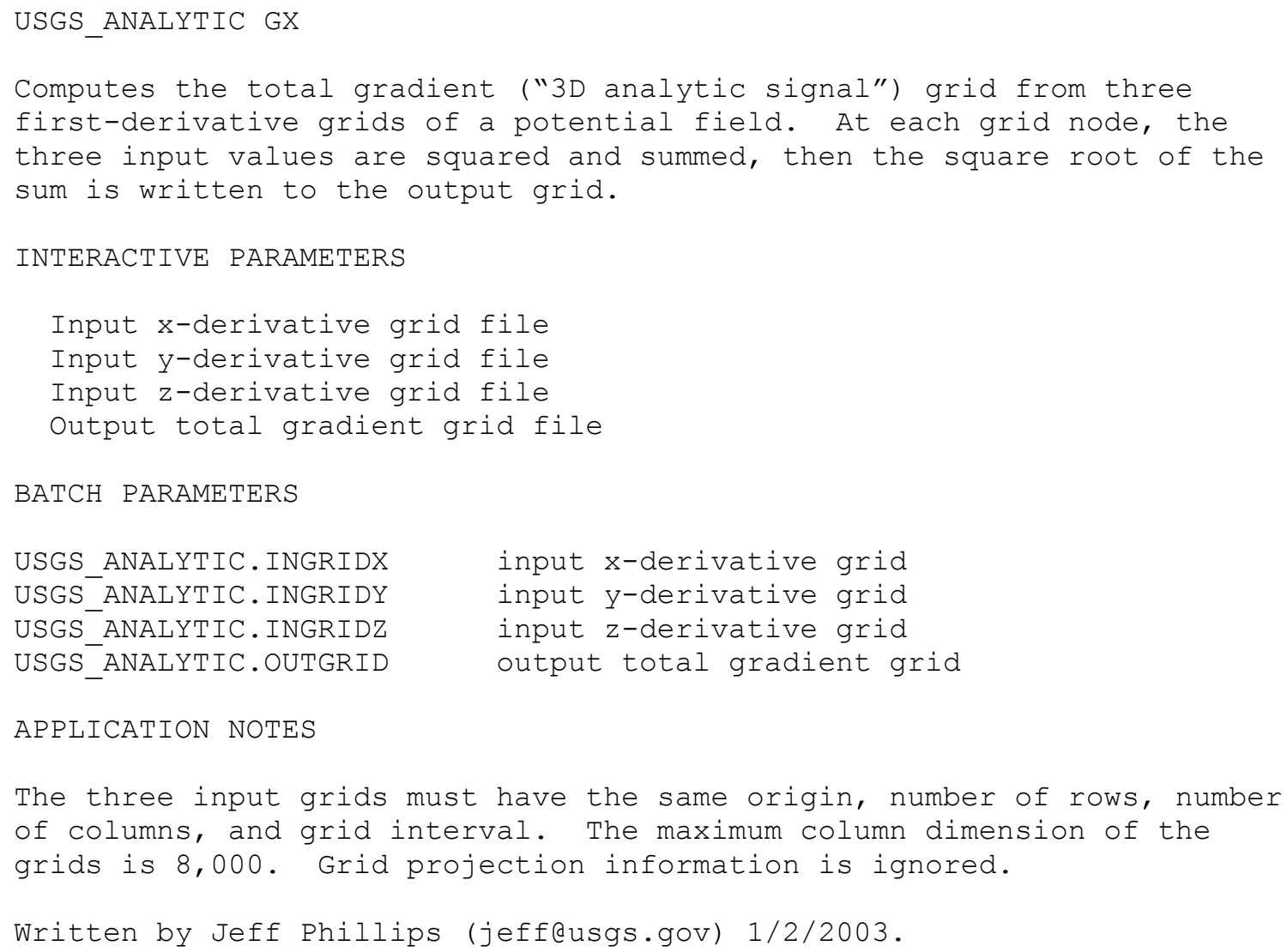




\section{USGS_BPFIL}

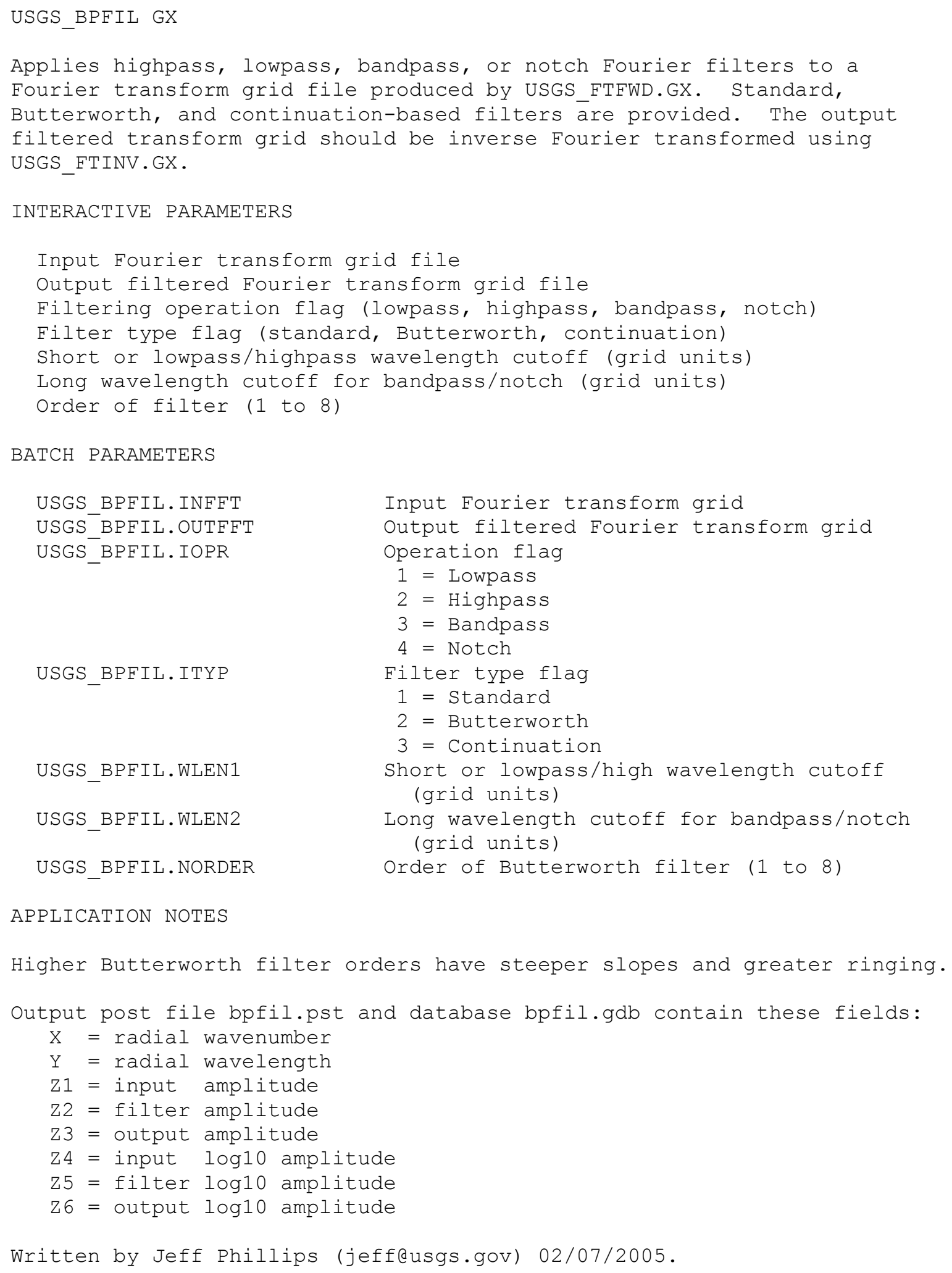

Written by Jeff Phillips (jeff@usgs.gov) 02/07/2005. 


\section{USGS_CTFIL}

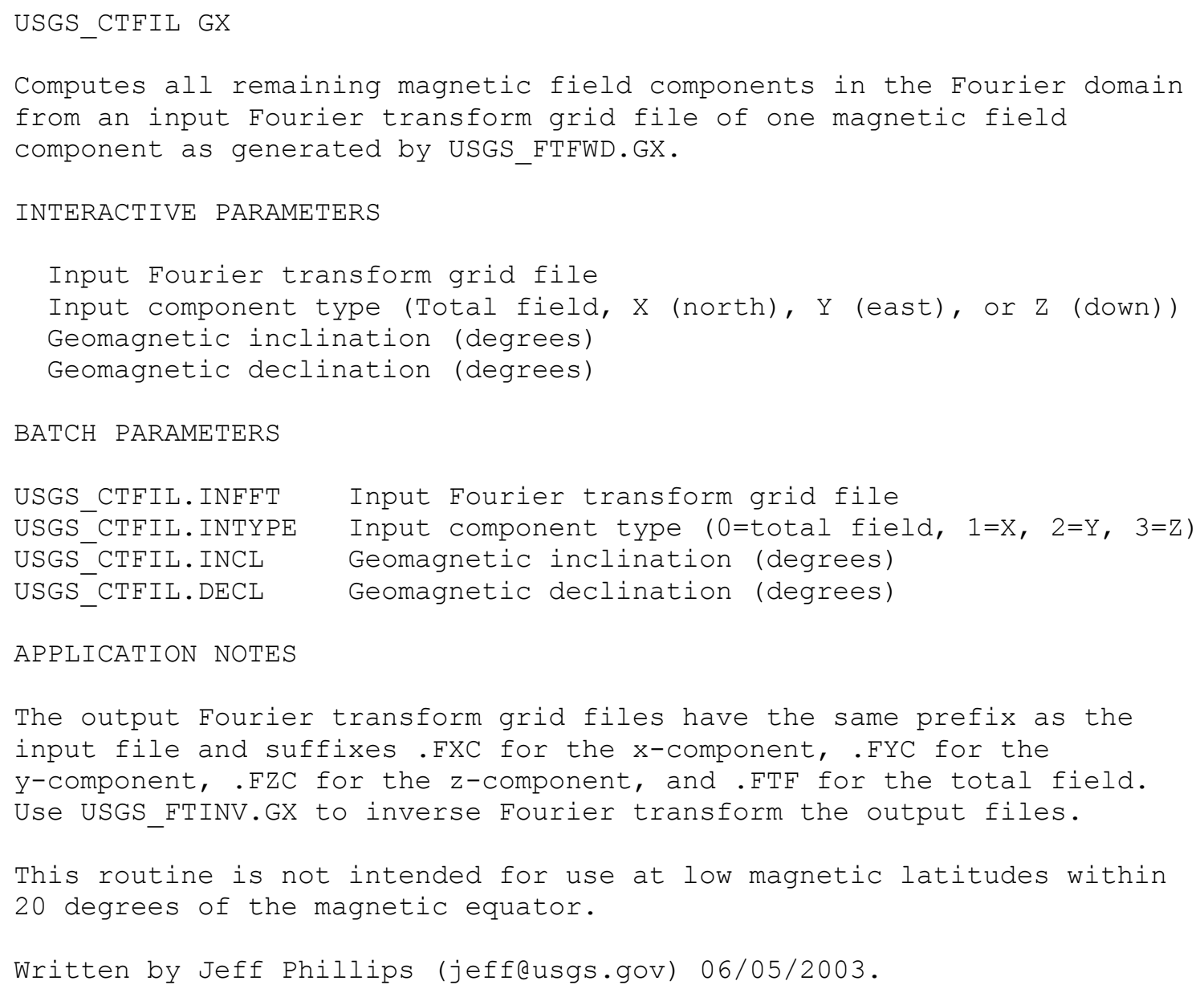




\section{USGS_CURV4}

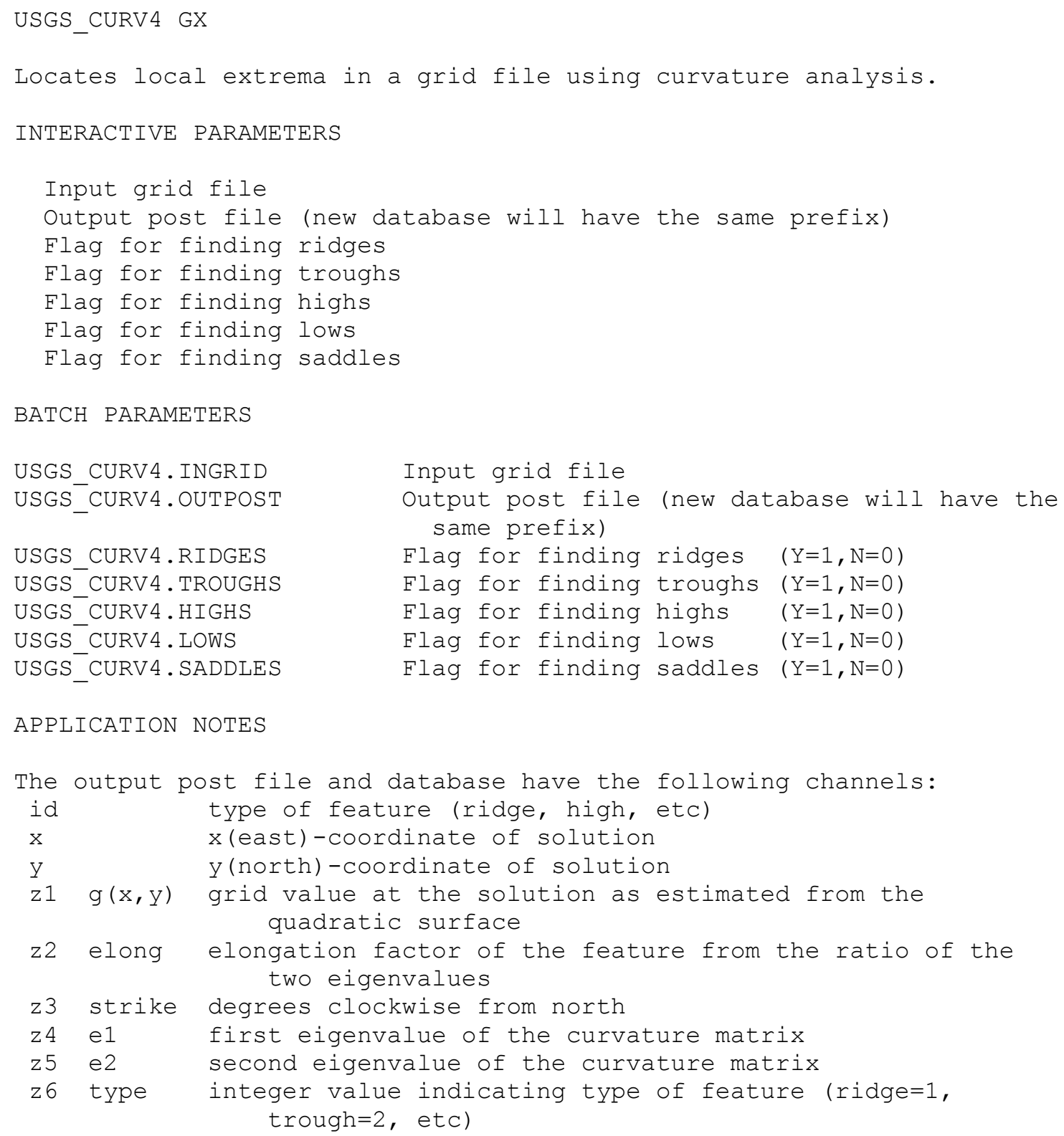




\section{USGS_DECIMATE}

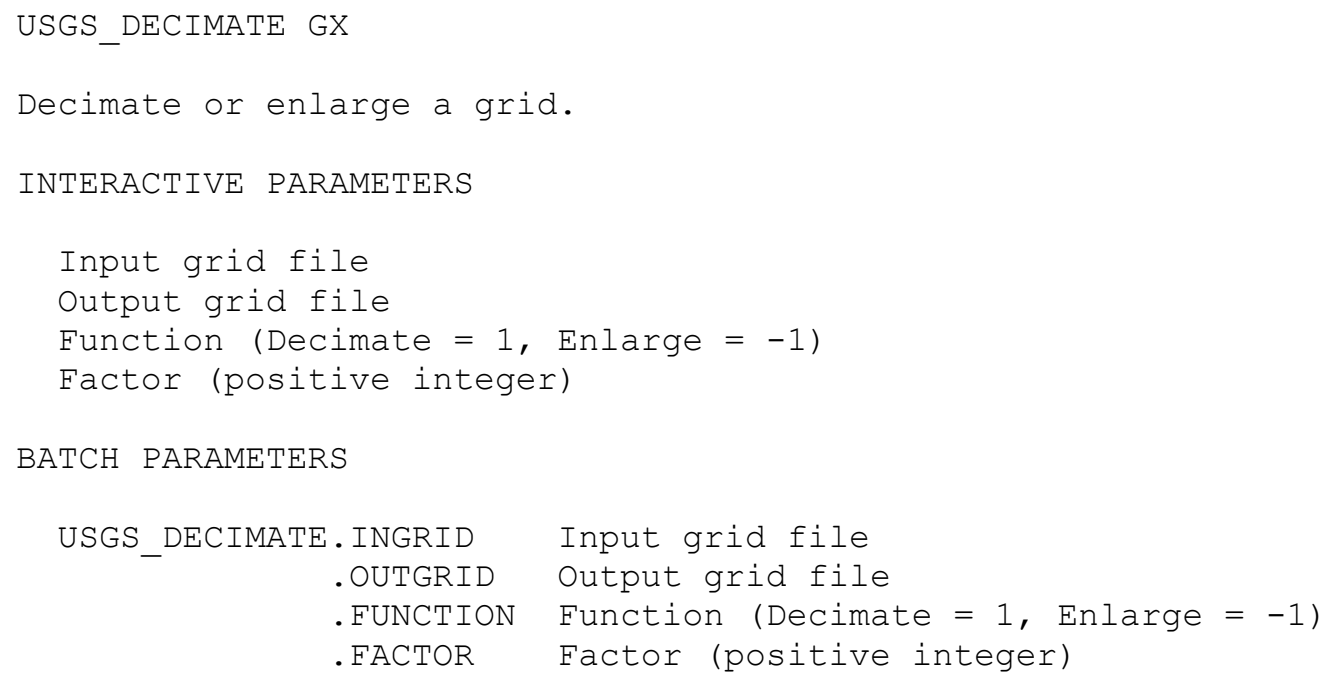




\section{USGS_DECOR}

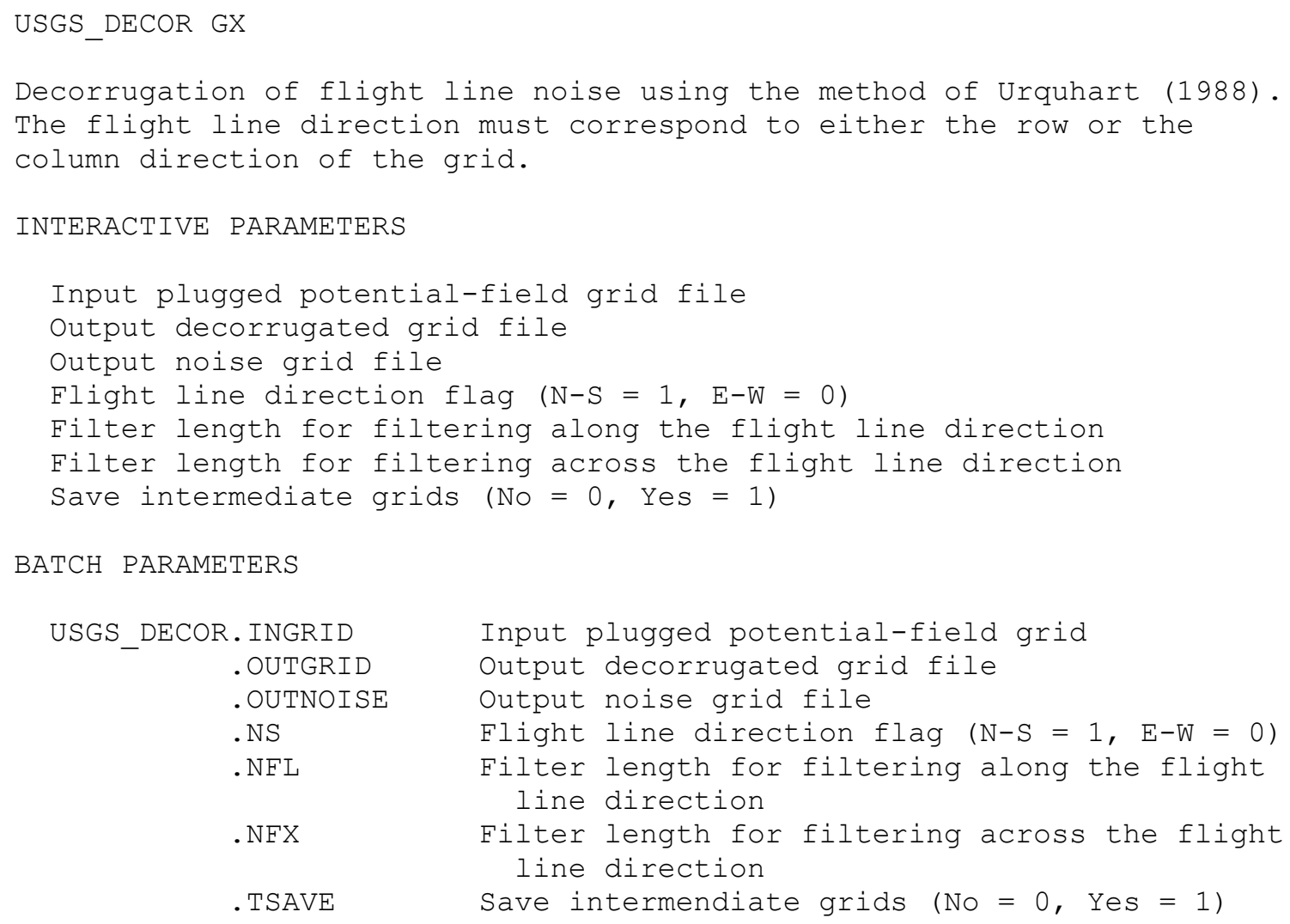

APPLICATION NOTES

Decorrugation of flight line noise in a grid using the method of Urquhart (1988). The flight line direction must correspond to either the row or the column direction of the grid. The algorithm is as follows:

1. The input grid USGS_DECOR. INGRID is checked for dummy values.

1a. For north-south flight lines the input grid is transposed to DECORO.TMP.

2. A Blackman filter (Oppenheim and Schafer, 1975, p.242) is used to lowpass filter the input grid (or DECORO.TMP) along the flight line direction, with the result stored in grid DECOR1.TMP.

2a. The highpass component along the flight lines, DECOR2.TMP, is computed as the difference between the input grid (or DECORO.TMP) and DECOR1. TMP.

3. DECOR1.TMP is transposed to DECOR3.TMP.

4. DECOR3.TMP is lowpass filtered perpendicular to the flight line direction using a different Blackman filter, with the result stored in DECOR4.TMP. This step is intended to remove the corrugations. 


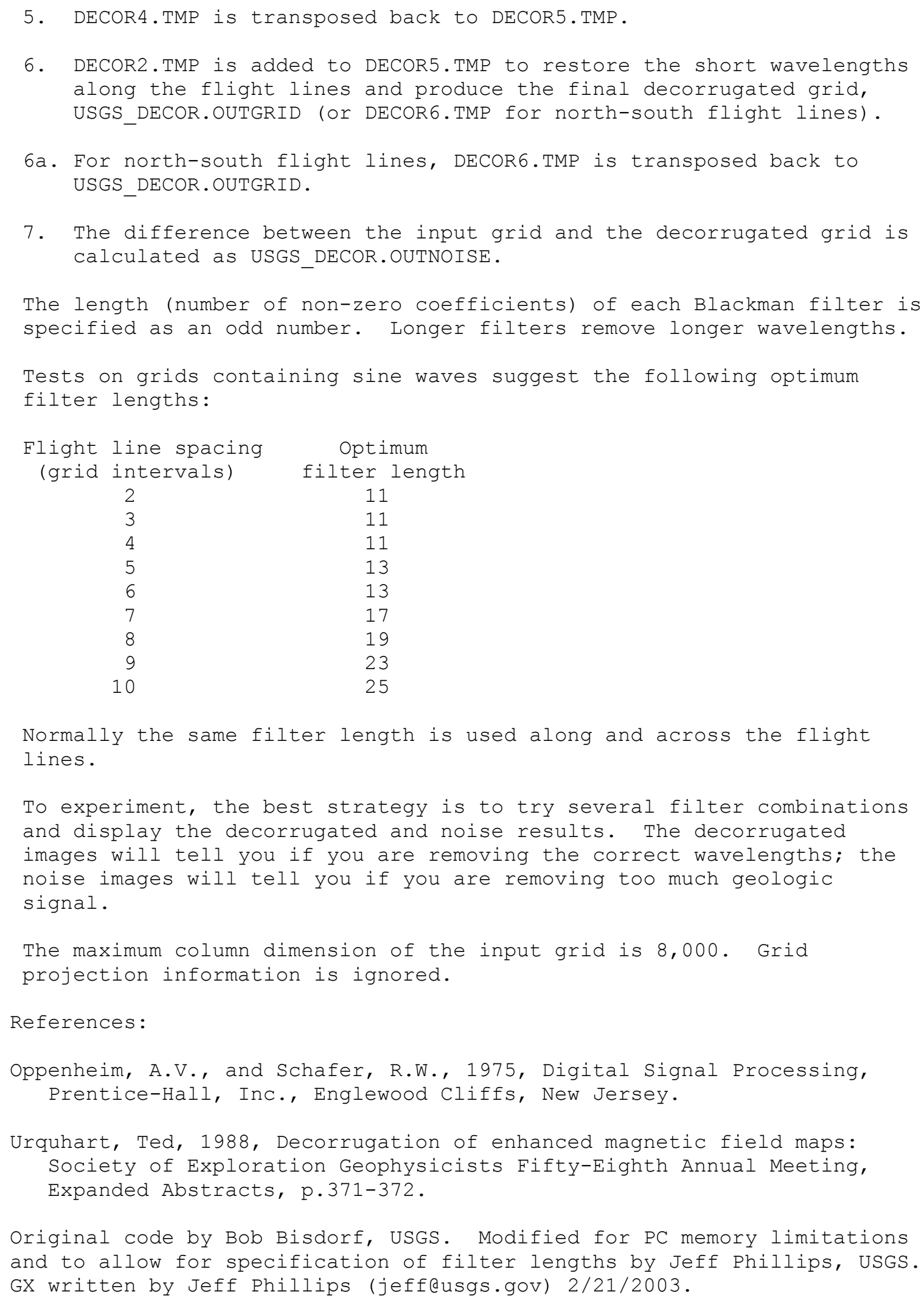

Original code by Bob Bisdorf, USGS. Modified for PC memory limitations and to allow for specification of filter lengths by Jeff Phillips, USGS. GX written by Jeff Phillips (jeff@usgs.gov) 2/21/2003. 


\title{
USGS_DIGVECS
}

\author{
USGS_DIGVECS GX \\ Digitize vectors from a map and place them in an ASCII XYZ file. \\ INTERACTIVE PARAMETERS
}

Output ASCII XYZ File

Append or Overwrite

Grid name

Line Thickness

Line Color

BATCH PARAMETERS

$\begin{array}{ll}\text { USGS_DIGVECS.XYZ } & \text { output ASCII XYZ file name } \\ \text { USGS_DIGVECS.APPEND } & \text { overwrite file }=0 ; \text { append to output = } 1 \\ \text { USGS_DIGVECS.GRID } & \text { grid name } \\ \text { USGS_DIGVECS.LINETHICK } & \text { line thickness (mm) } \\ \text { USGS_DIGVECS.LINECOLOR } & \text { line color }\end{array}$

APPLICATION NOTES

If the output file does not exist, you must specify overwrite mode. If the file does exist and you specify overwrite mode, you will be asked to confirm the overwrite.

If no grid is specified, only LINE, $X, Y$ coordinates will be placed in the output file. If a grid is specified, LINE,X, Y and Z values will be placed in the output file. A header record in the output file identifies the channel names. To use this header record when importing the XYz file into a database, blank out the 'Import template' field.

A map group corresponding to the output file name prefix will be used to hold the vectors drawn on the map.

Multi-point vectors are digitized using the left mouse button. The right mouse button is used to terminate a vector (using the "Done" menu item) and start a new vector. Digitizing is terminated by using the right mouse button and selecting "Done" twice in a row. The "Cancel" menu item is equivalent to the "Done" menu item, it does not remove the digitized vector from the file or the map. The "Exit Menu" menu item will cancel the menu and return to digitizing the current vector.

Because the map cannot be redrawn inside a GX, the digitized vectors will not be drawn until the GX is exited. For the same reason, the remaining right mouse menu items won't work properly.

Written by Jeff Phillips (jeff@usgs.gov) 2/9/99, modified 3/21/02. 


\section{USGS_DVALTEST}

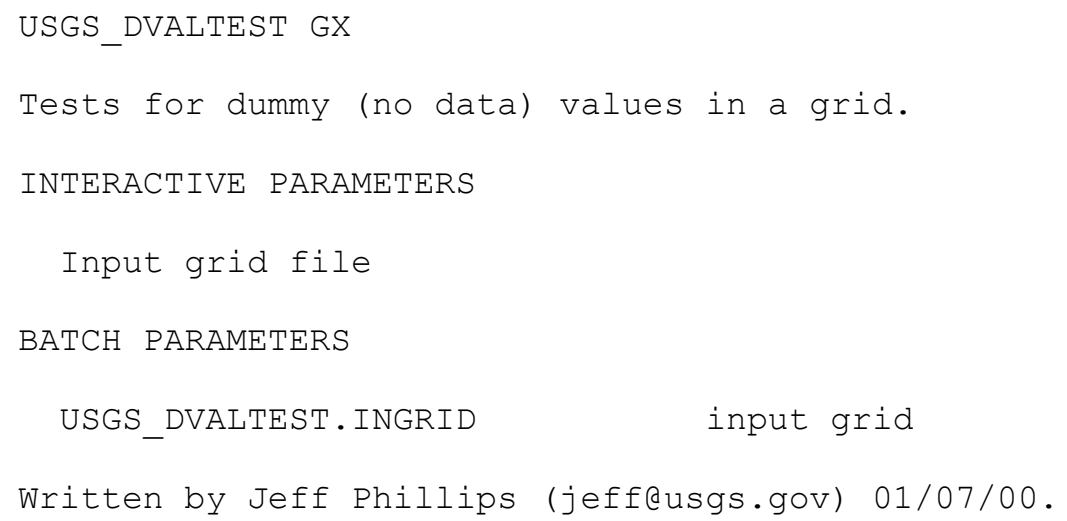




\begin{tabular}{|c|c|}
\hline & x-component grid file \\
\hline INGRIDHY & $\begin{array}{l}\text { Input Hilbert transform y-component grid } \\
\text { file }\end{array}$ \\
\hline - INGRIDHYX & $\begin{array}{l}\text { Input } x \text {-derivative of Hilbert transform } \\
\text { y-component grid file }\end{array}$ \\
\hline INGRIDHYY & $\begin{array}{l}\text { Input y-derivative of Hilbert transform } \\
\text { y-component grid file }\end{array}$ \\
\hline . INGRIDHYZ & $\begin{array}{l}\text { Input z-derivative of Hilbert transform } \\
\text { y-component grid file }\end{array}$ \\
\hline . SIMIN & Minimum structural index \\
\hline IWIN & Window size \\
\hline . ITYPE & Type of depth error to use \\
\hline MAXERR & Maximum error in depth \\
\hline MAXDIST & $\begin{array}{l}\text { Maximum horizontal distance of solutions } \\
\text { from center of window }\end{array}$ \\
\hline . MAXDEPTH & Maximum depth of solutions \\
\hline UTPOST & Output post file \\
\hline
\end{tabular}

\section{METHOD DESCRIPTION}

USGS_EULERAVE.GX estimates the parameters of isolated magnetic sources from gridded magnetic anomaly data using Euler's equation (Reid and others, 1990). Analysis can be done on any combination of the magnetic anomaly grid and its Hilbert transform components (Nabighian and Hansen, 2001). Successful results are averaged for each window.

USGS_SETEULER.GX is used to generate the Hilbert transform component grids and the horizontal and vertical derivative grids used by USGS EULERAVE.GX. Optional input grids include the elevation of the observation surface and the elevation of a reference surface, such as the topography or the seismic basement, that represents the top of the shallowest expected sources.

Because the depth and structural index cannot be estimated simultaneously (Barbosa and others, 1999), analysis is done in two stages (Phillips, 2002) by passing a window over the data grids. First an attempt is made to estimate a source location and background constant (xo, yo, zo,K) from the data within the window using an initial structural index (usually zero). If the attempt is successful, and the resulting source lies on or below the reference surface, the source is retained as valid. If the attempt is successful, but the resulting source lies above the reference surface, the source is moved down to the reference surface and a solution for the horizontal location, structural index, and background constant (xo,yo, si, K) is attempted. If this second stage is successful, the source on the reference surface is retained as valid. If the initial structural index is set to zero (the default), then the combined solutions on and below the reference surface will represent the shallowest possible magnetic basement surface. For a more traditional Euler analysis, the initial structural index can be specified and the reference surface can be set equal to the observation surface.

\section{APPLICATION NOTES}

All input grids must have the same origin, number of rows, number of columns, and grid intervals. The maximum column dimension of the input grids is 8,000 . 
Leave the input observation surface grid file entry blank to use the constant observation surface elevation.

Leave the input minimum depth surface grid file entry blank to use the constant minimum depth surface elevation. Examples of minimum depth surfaces are the topography, bathymetry, or seismic basement.

Leave the Hilbert transform component grid file entries blank to force conventional Euler depth analysis.

The input potential-field grid entry can be left blank to generate solutions due solely to the Hilbert transform components.

If the maximum horizontal distance entry is left blank, a value of (window_size-1)* $(|d x|+|d y|) / 4$ is used, where $d x$ and dy are the grid intervals in the east and north directions respectively.

Output of the program is contained in the specified binary post file and in a database with the same name as the post file, both containing the following fields:

id blank

$x$ the average $x$ (east) coordinate of the source

$y$ the average $y$ (north) coordinate of the source

zl the average elevation (positive upward) of the source

z2 the average estimated elevation error (either absolute or as \% of depth)

z3 a dummy value for the strike of the feature

z4 the information index $=z 5 / z 2$

z5 the number of solutions in the average

z6 the average estimated structural index of the source

Output horizontal and vertical units are the same as the input horizontal grid units.

ADDITIONAL APPLICATION NOTES FROM BEN DRENTH

Initial Considerations

1. The Euler deconvolution method is insensitive to magnetic remanence and inclination effects, so it is preferable to use a standard total-field anomaly grid (i.e., not reduced-to-pole).

2. This implementation is not affected by holes in the total-field anomaly grid. It is preferable that the grid not be plugged, so that spurious solutions in plugged regions are avoided.

3. The total-field anomaly grid must be free of excessive flight-line noise (or other types of obvious noise).

4. Before starting, decide on a window size or an appropriate range of window sizes to test. Window size should be ideally chosen large enough so that full anomalies just fit within the window, yet small enough so that adjacent anomalies cannot contaminate that window. This can easily be examined using the ruler function in Oasis.

5. Window sizes are expressed in terms of grid spacing, so for a grid with a 400 meter interval, a window size of 25 would produce a window 10,000 meters on a side. If this is too small a window (see \#4), then re-grid the total-field anomaly grid to a larger interval in order to appropriately represent anomaly width using 
window sizes of 25 or less.

6. Before starting, make sure that you know the surface elevation and flight surface for the total-field anomaly grid. You will be making grids of each in the following steps.

Euler Setup

1. The total-field anomaly, ground surface elevation, and flight surface grids must all be perfectly coincident. In order to most easily achieve this, save the total-field anomaly grid to a database. Next, create new database channels for the ground and flight surface elevations and interpolate from the appropriate grids into those channels.

2. Make the following grids, using the same gridding parameters for each: total-field anomaly, ground elevation, and flight surface.

3. Run the Euler setup GX. The input grid file is the totalfield anomaly grid from the previous step. Use the defaults for all of the listed parameters.

Euler Depth Analysis

1. This is mostly self-explanatory as most of the fields are automatically populated after running the Euler setup. However, there are a few tricks. After specifying the observation (flight) surface and minimum depth (ground elevation) surface, enter the factor for elevation units/horizontal units. This factor is 1000 if the grid units are in kilometers and the elevation units are in meters.

2. The structural index values for magnetic sources are listed below. An advantage of using an index of 0 is that it will yield the minimum possible depths to the tops of the magnetic sources. An index of 1 will yield the maximum possible depths to the tops of most geological magnetic sources, so these two sets of solutions can be compared in a meaningful way.

\begin{tabular}{|c|c|c|}
\hline$\frac{\text { Structural }}{\text { Index }}$ & Geologic Model & Depth Type \\
\hline 0 & high-throw contact/fault & depth to top \\
\hline 1 & low-throw contact/fault & depth to top \\
\hline 2 & line source & depth to center \\
\hline 3 & sphere & depth to center \\
\hline
\end{tabular}

3. Run the program with different window sizes and compare the solutions. A 25 percent error gives a nicely large number of solutions for later gridding. Always set the maximum horizontal distance parameter to 0 , which suppresses horizontal location errors. The maximum depth should be set to a very large number, so that there is no risk of excluding deeper solutions.

Manipulating the Results

1. If an observation surface grid has been used, the depth solutions are listed in horizontal grid units above sea level, so these must be converted to depths by being subtracted from the surface elevation. An elevation grid can be sampled into the database for this purpose. Negative depth solutions are commonly present in the database; these can be masked out prior to gridding.

2. Gridding the depths at 3-6 times the original total-field anomaly grid interval is pretty good for displaying results over a large 
area. Multiply the depth grid by -1 so that "cold" color solutions appear "deep" on a color shaded image map.

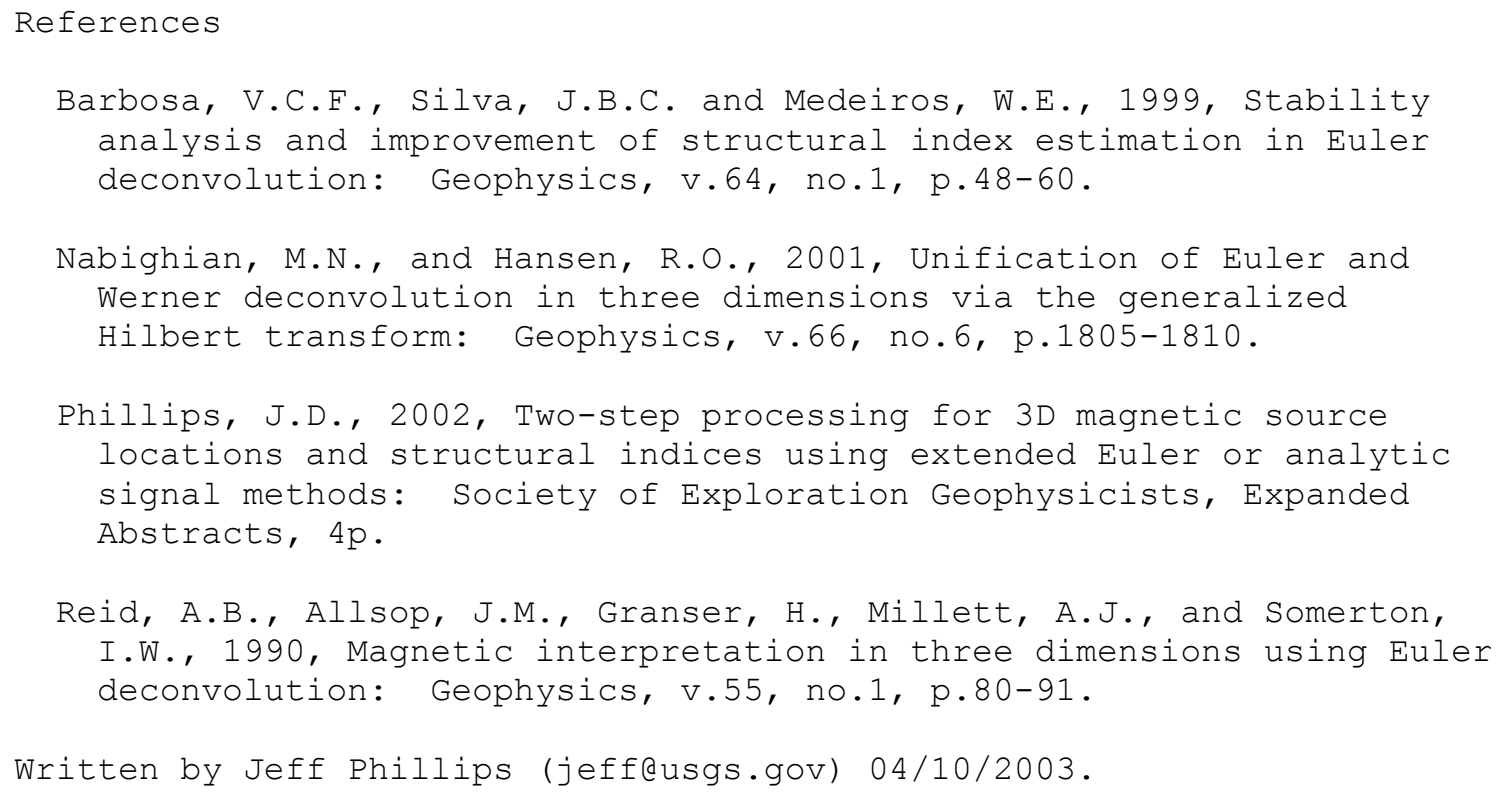




\section{USGS_FTFIL}

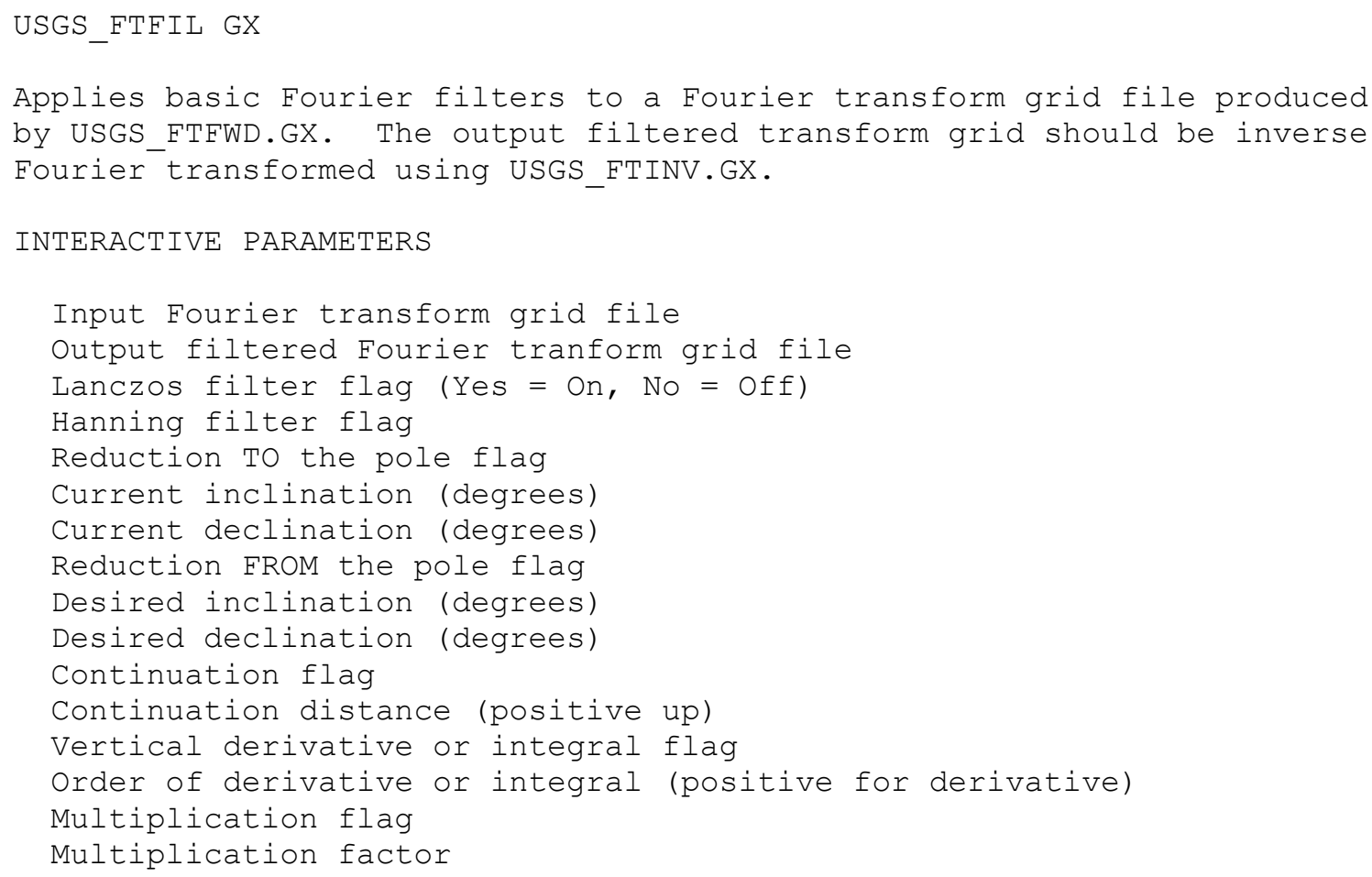

USGS FTFIL.INFFT

USGS FTFIL.OUTFFT

USGS_FTEIL.ILANC

USGS FTFIL. IHANN

USGS FTFIL. IPOLE

USGS FTFIL.AINCL

USGS FTFIL.ADECL

USGS FTFIL.IFROM

USGS FTFIL.BINCL

USGS FTFIL.BDECL

USGS FTFIL.ICONT

USGS FTFIL.HEIGHT

USGS FTFIL.IVERT

USGS_FTFIL.RORDER

USGS_FTFIL.IMULT

USGS_FTFIL.AMULT

APPLICATION NOTES

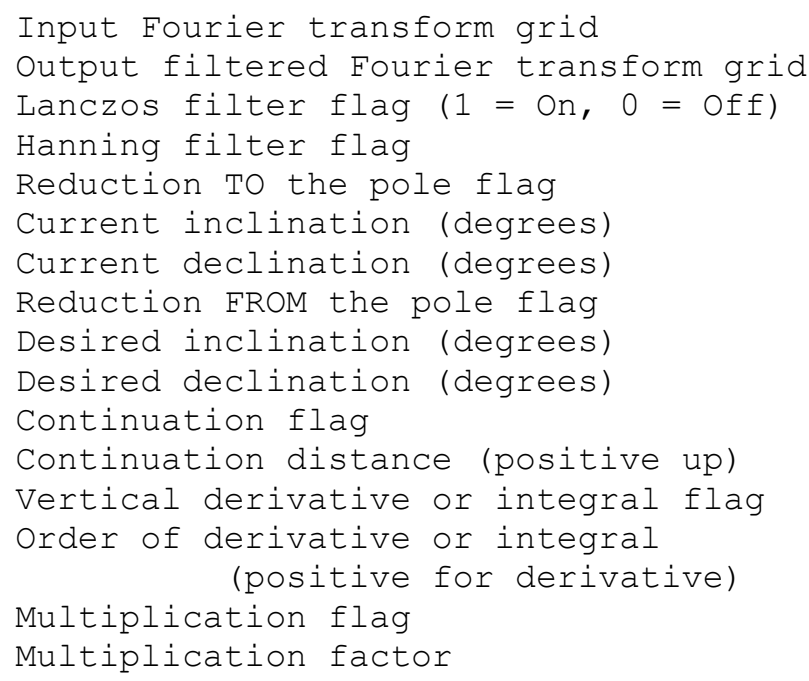

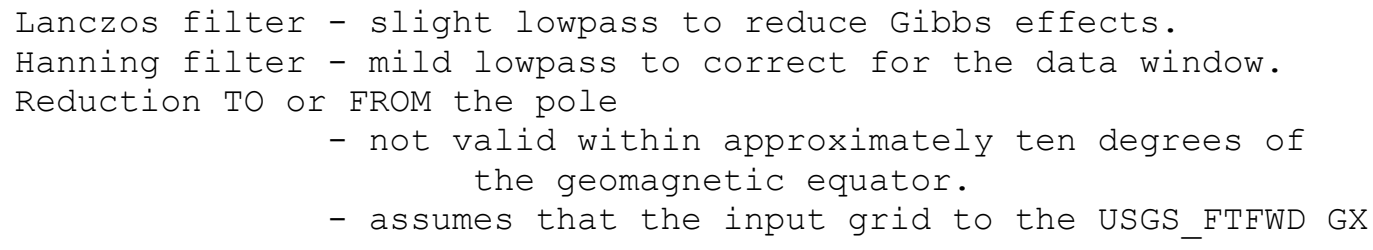




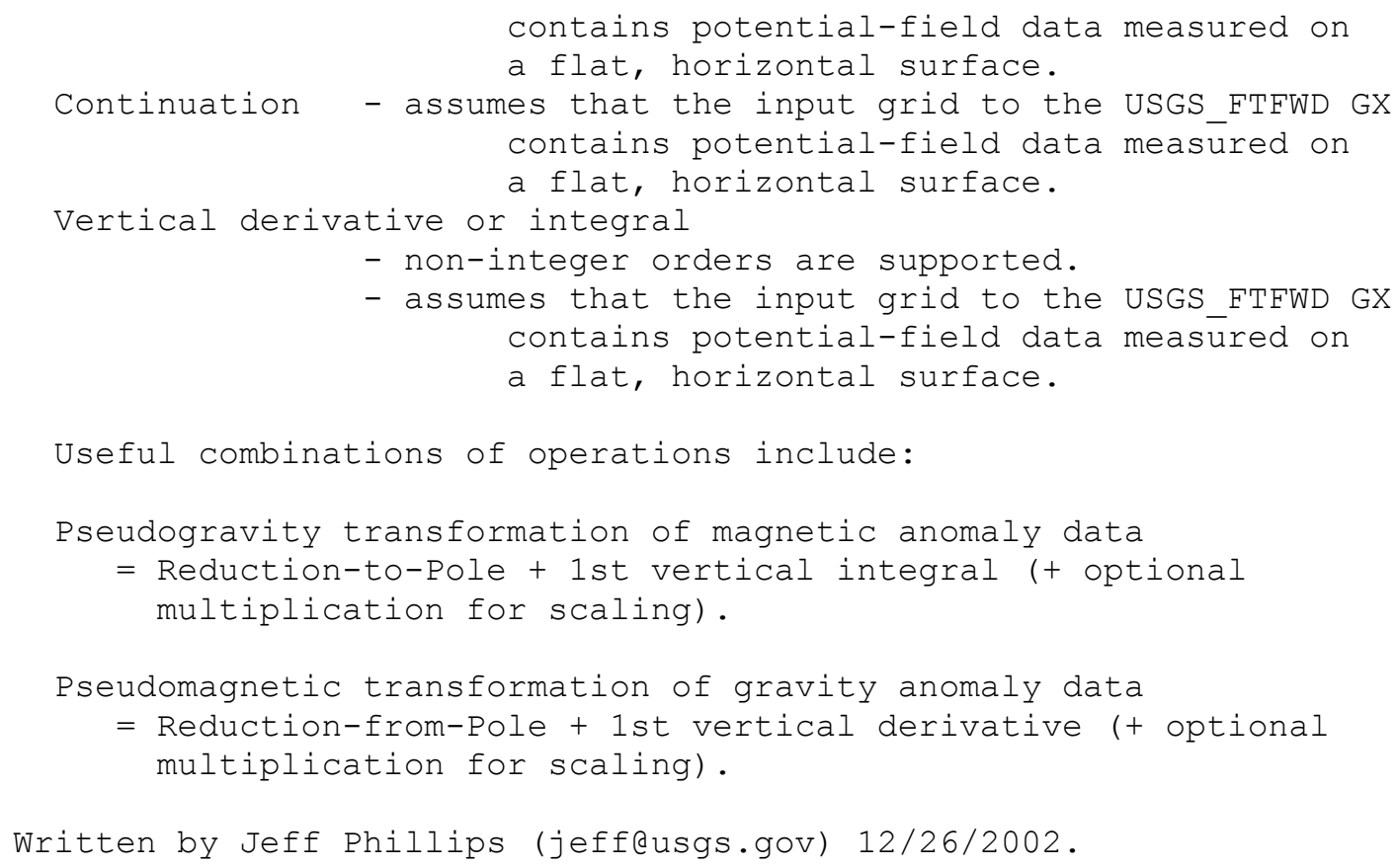




\section{USGS_FTFWD}

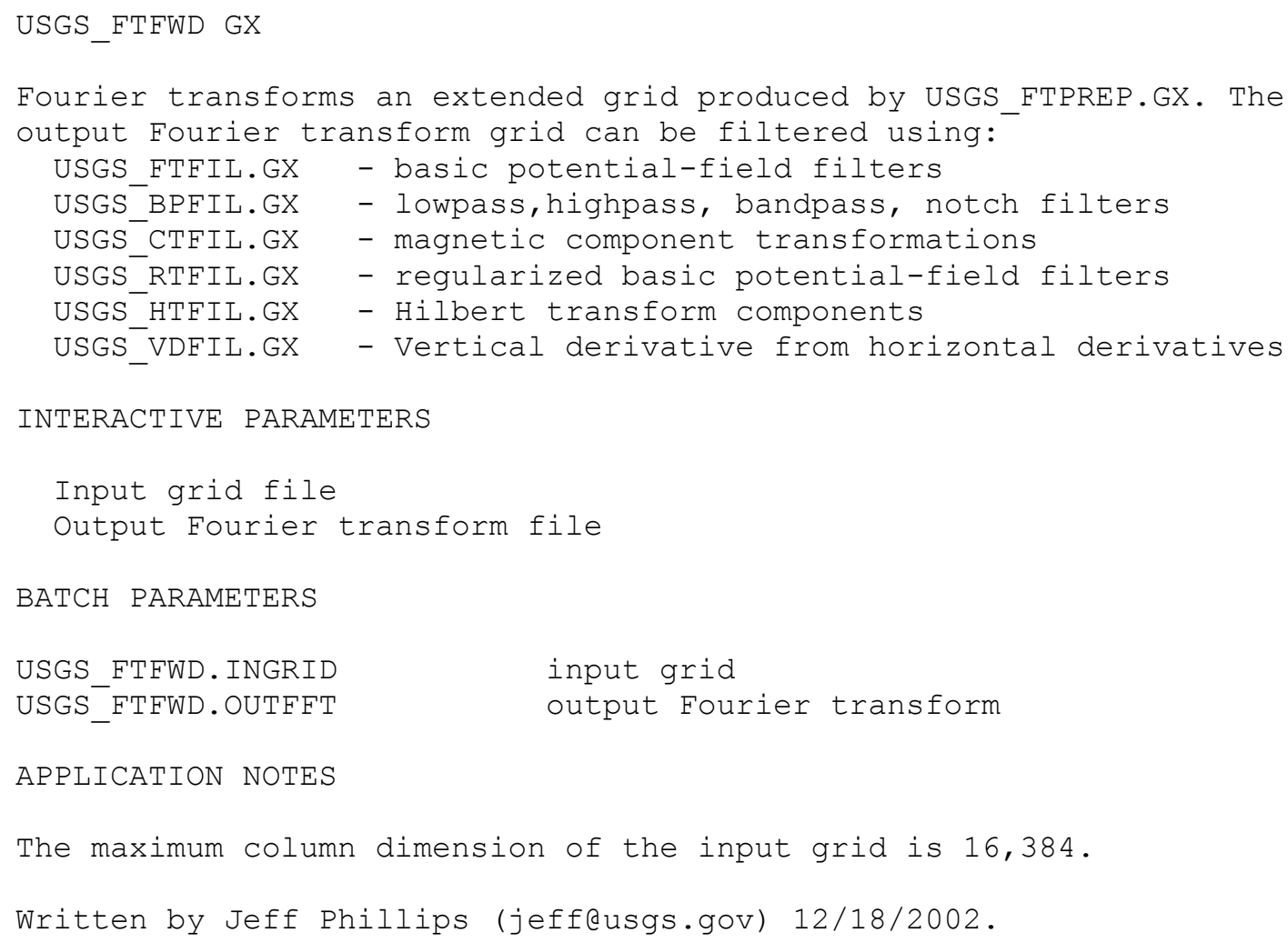




\section{USGS_FTINV}

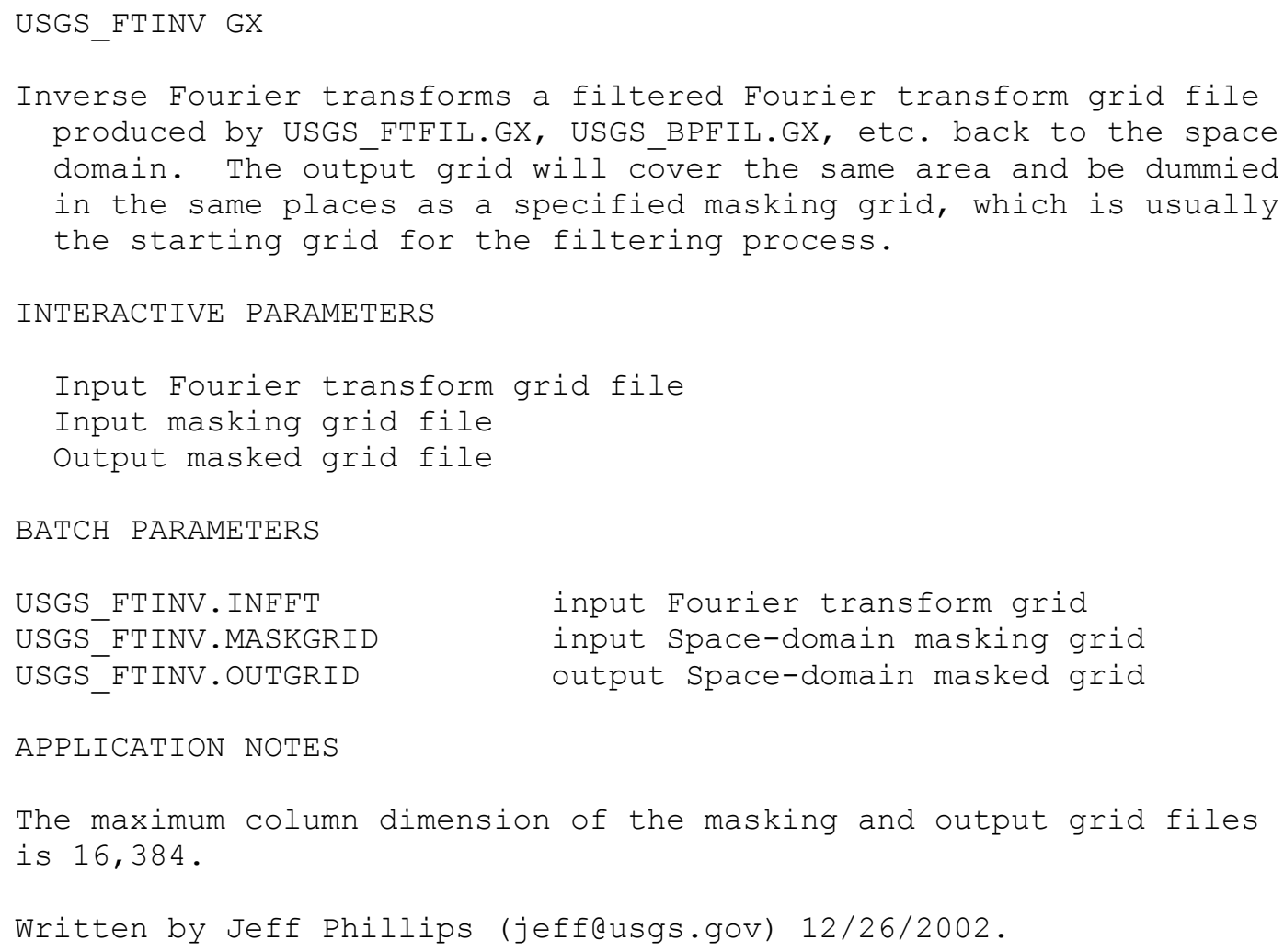




\section{USGS_FTPREP}

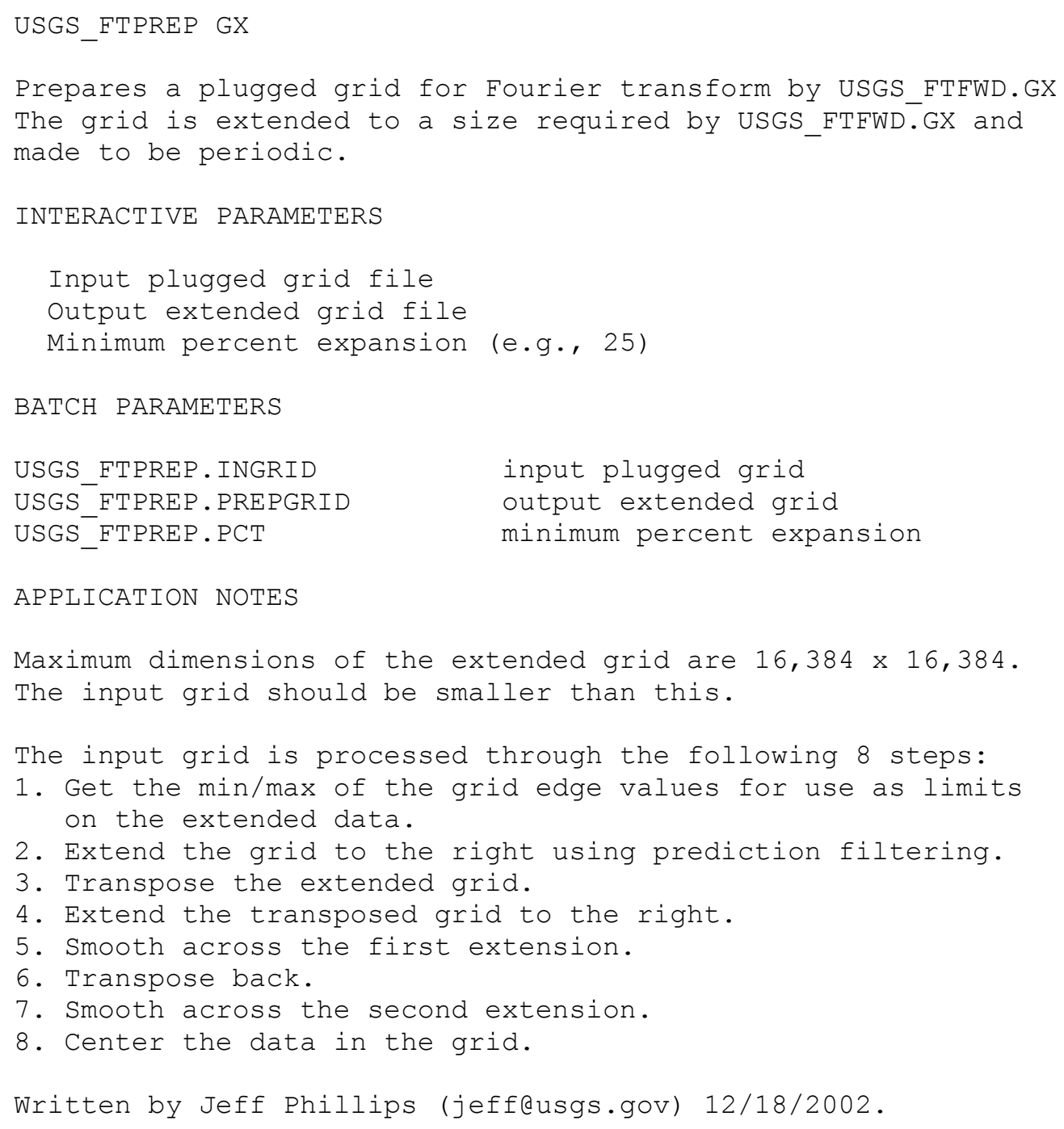




\section{USGS_GRADCOMP}

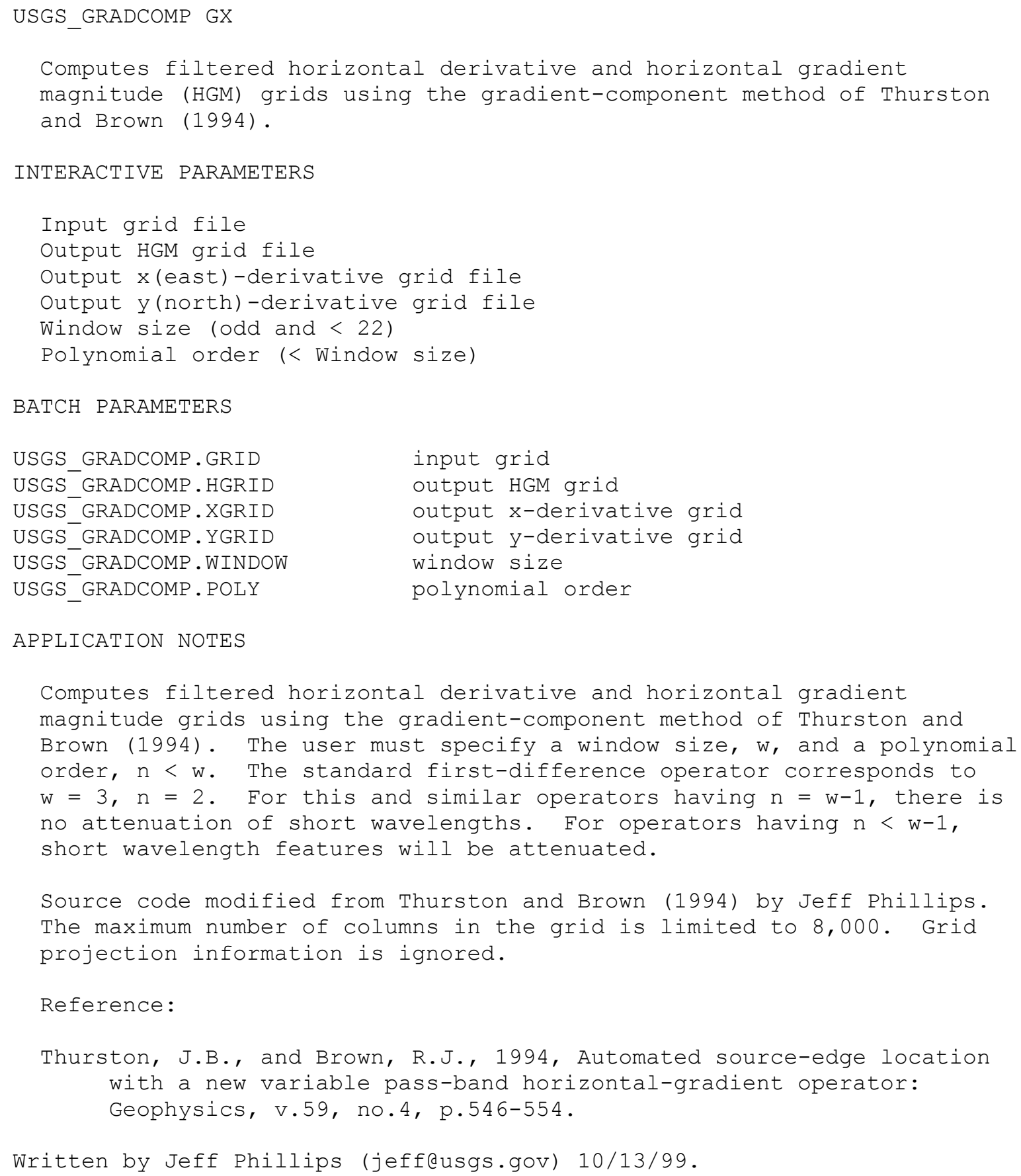

APPLICATION NOTES

Computes filtered horizontal derivative and horizontal gradient magnitude grids using the gradient-component method of Thurston and Brown (1994). The user must specify a window size, w, and a polynomial order, $\mathrm{n}<\mathrm{w}$. The standard first-difference operator corresponds to $\mathrm{w}=3, \mathrm{n}=2$. For this and similar operators having $\mathrm{n}=\mathrm{w}-1$, there is no attenuation of short wavelengths. For operators having $\mathrm{n}<\mathrm{w}-1$, short wavelength features will be attenuated.

Source code modified from Thurston and Brown (1994) by Jeff Phillips. The maximum number of columns in the grid is limited to 8,000. Grid projection information is ignored.

Reference:

Thurston, J.B., and Brown, R.J., 1994, Automated source-edge location with a new variable pass-band horizontal-gradient operator: Geophysics, v.59, no.4, p.546-554.

Written by Jeff Phillips (jeff@usgs.gov) 10/13/99. 


\section{USGS_GRADXYH}

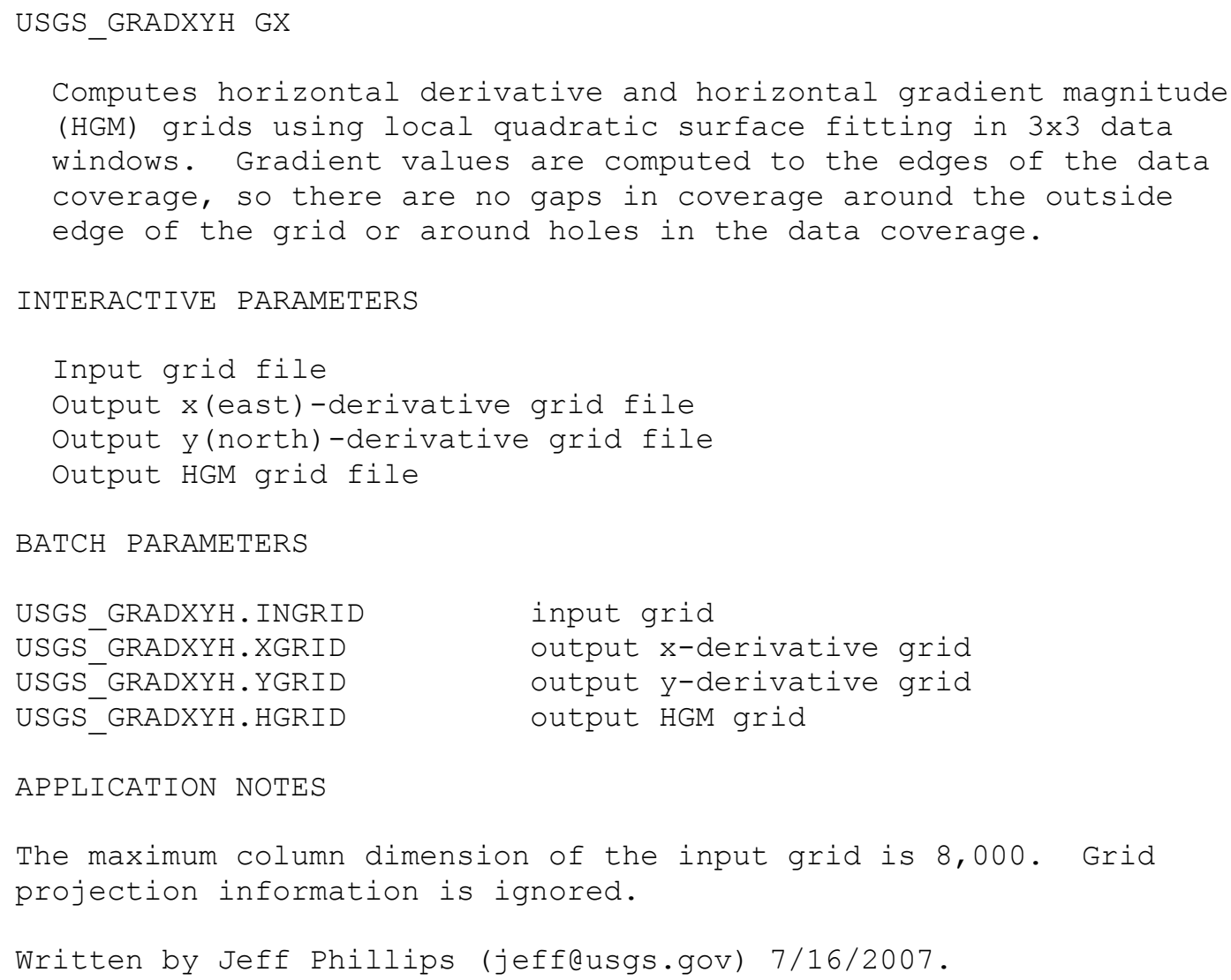




\section{USGS_GRIDMASK}

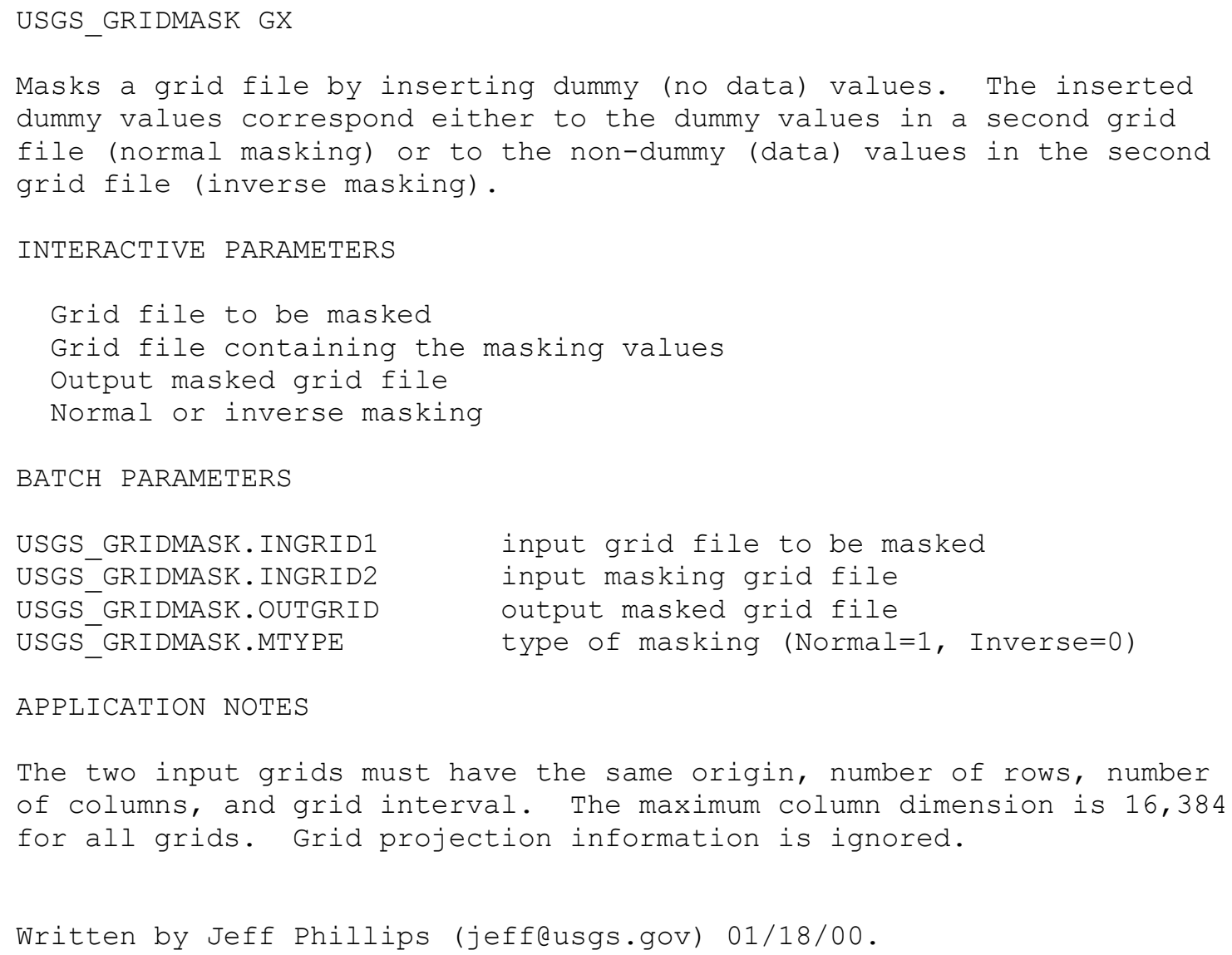




\section{USGS_GRIDPLUG}

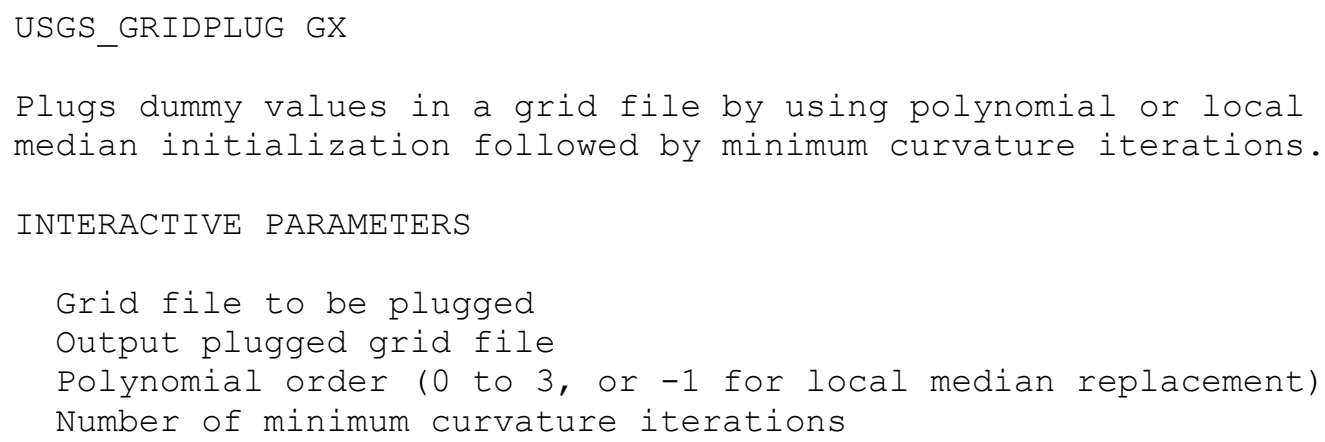




\section{USGS_GRIDPROX}

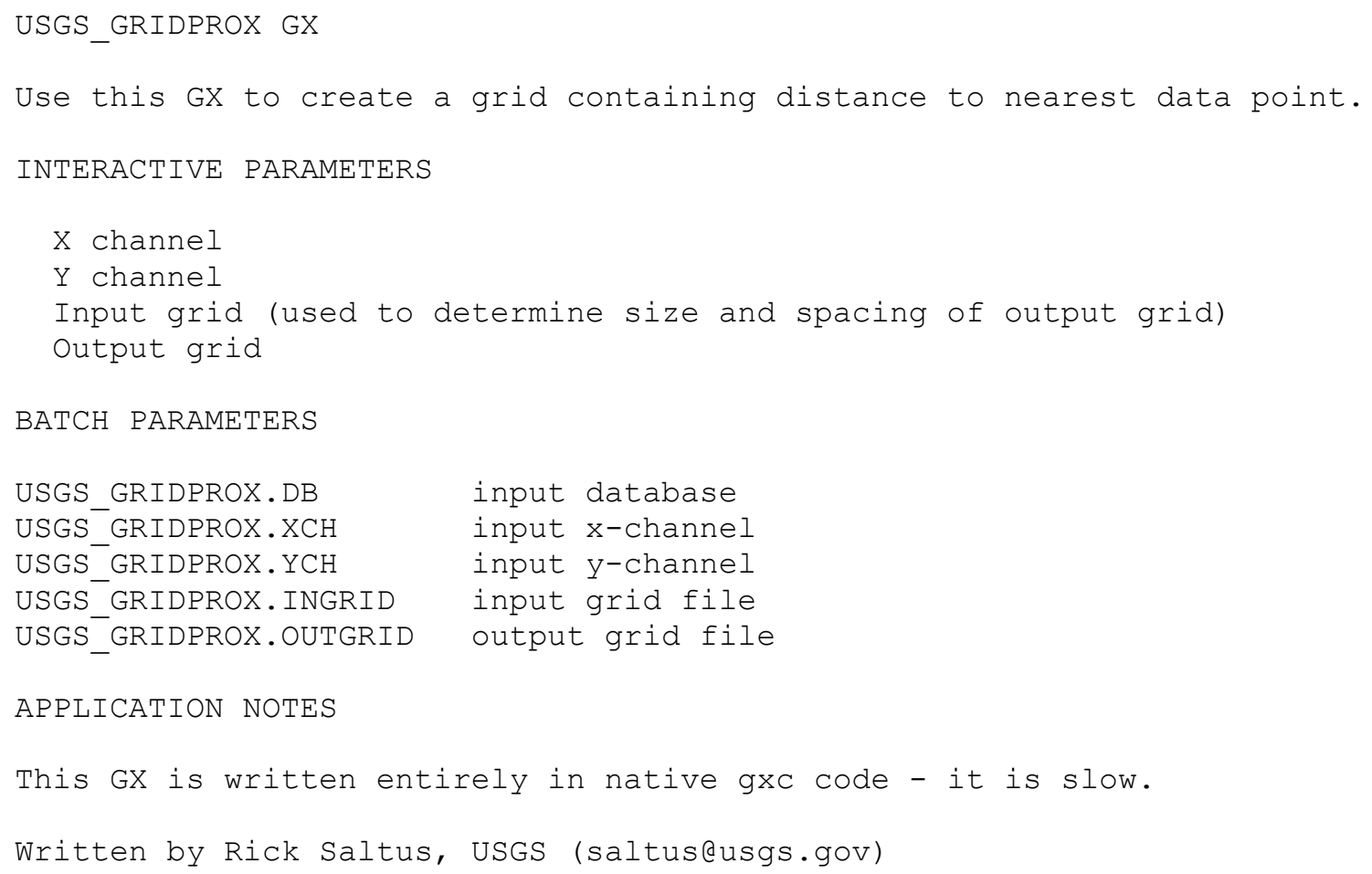




\section{USGS_GRIDSAMP}

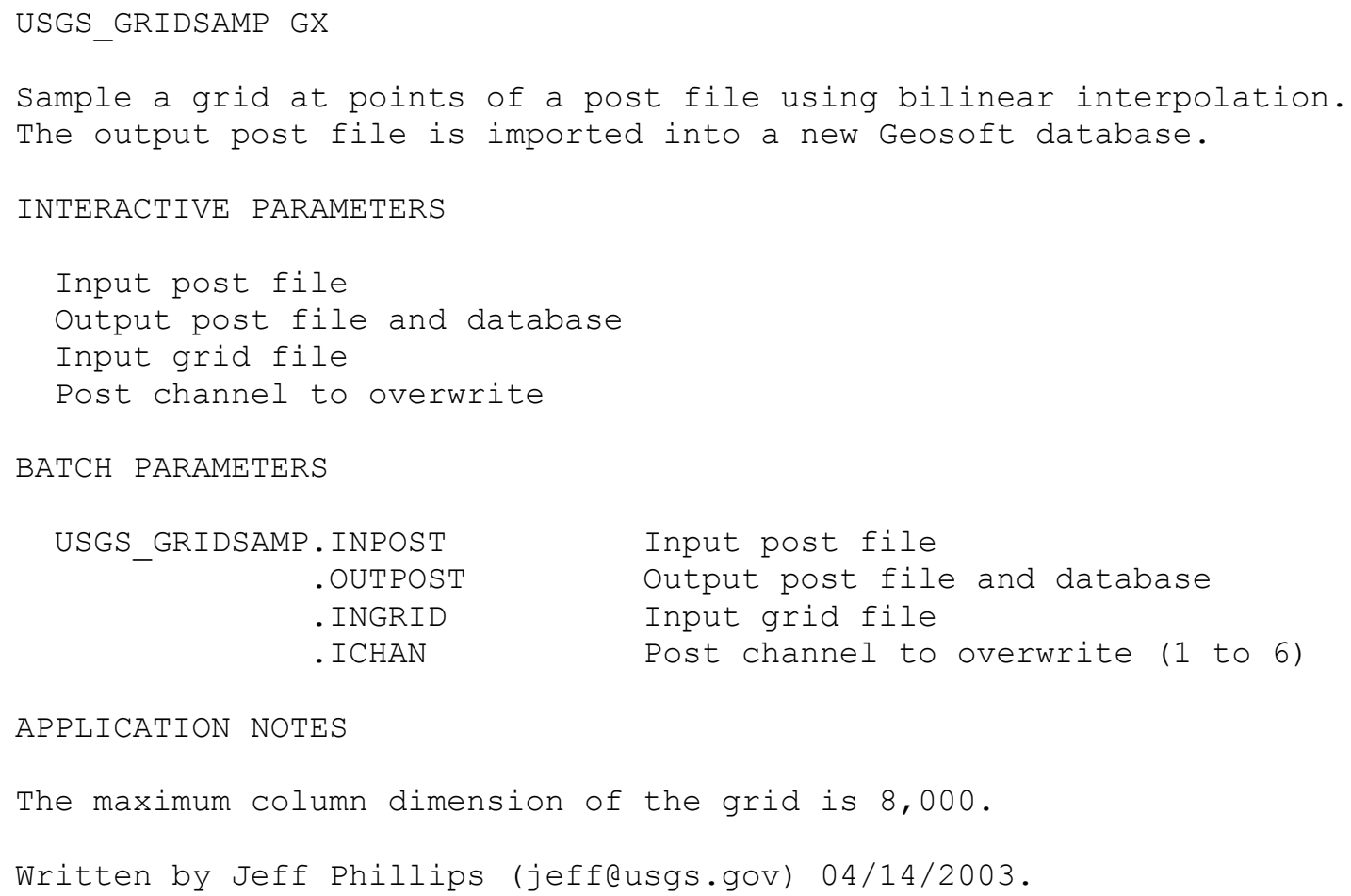




\section{USGS_HGRAD}

USGS_HGRAD.GX

This GX calculates the magnitude of the horizontal gradient of a grid. INTERACTIVE PARAMETERS

Input grid

Output horizontal gradient magnitude grid

BATCH PARAMETERS

USGS HGRAD.IN = Input grid

USGS_HGRAD.OUT = Output horizontal gradient magnitude grid

APPLICATION NOTES

The HORIZONTAL GRADIENT MAGNITUDE is the square root of the sum of the squares of the derivatives in the $x$ and $y$ directions:

hgrad $=\operatorname{sqrt}\left(d x x^{\star} d x+d y^{\star} d y\right)$

Written by Northwest Geophysical Associates for the USGS.

Requires licensed grid filtering. 


\section{USGS_HTFIL}

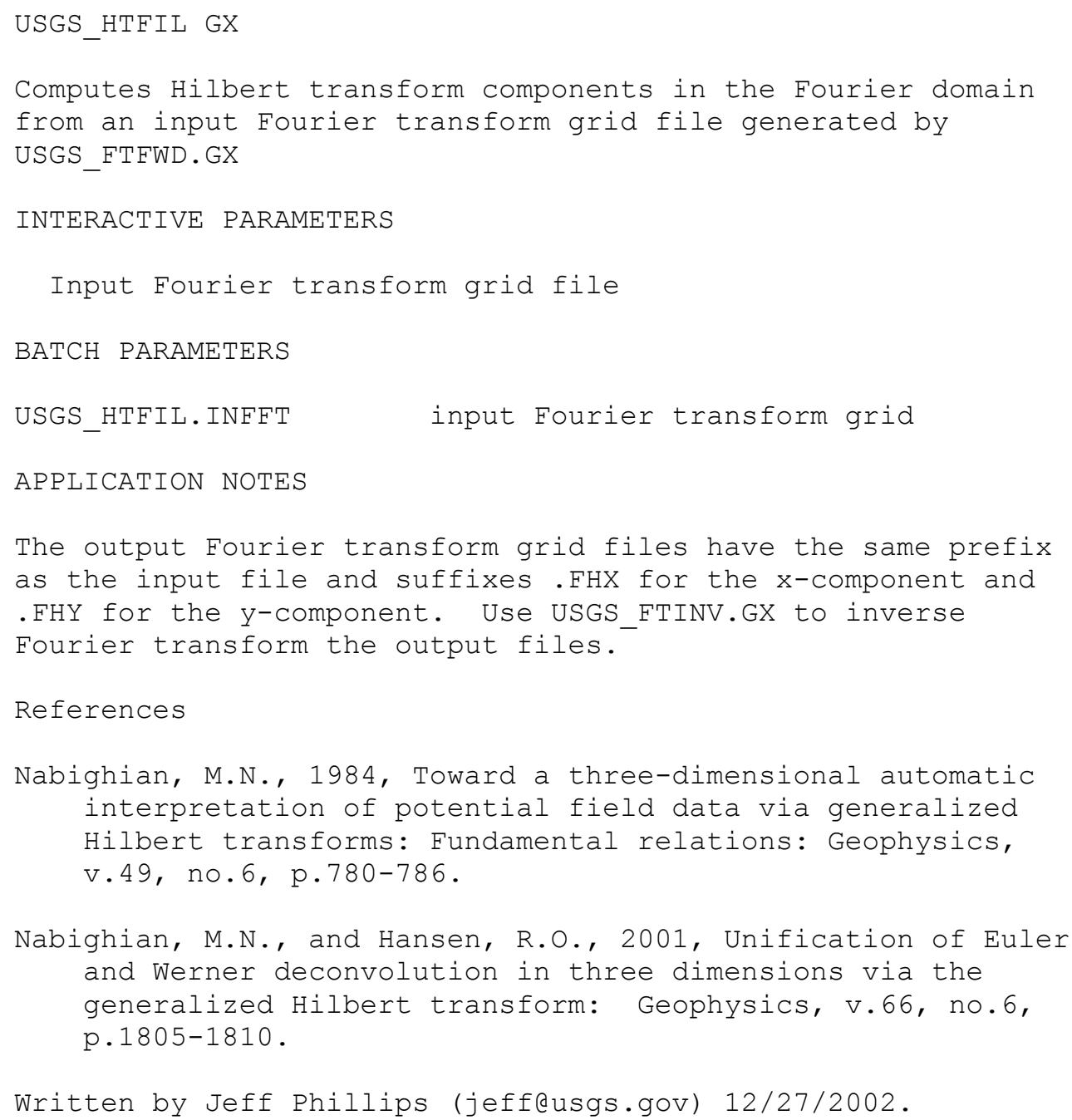




\section{USGS_IGRFPT}

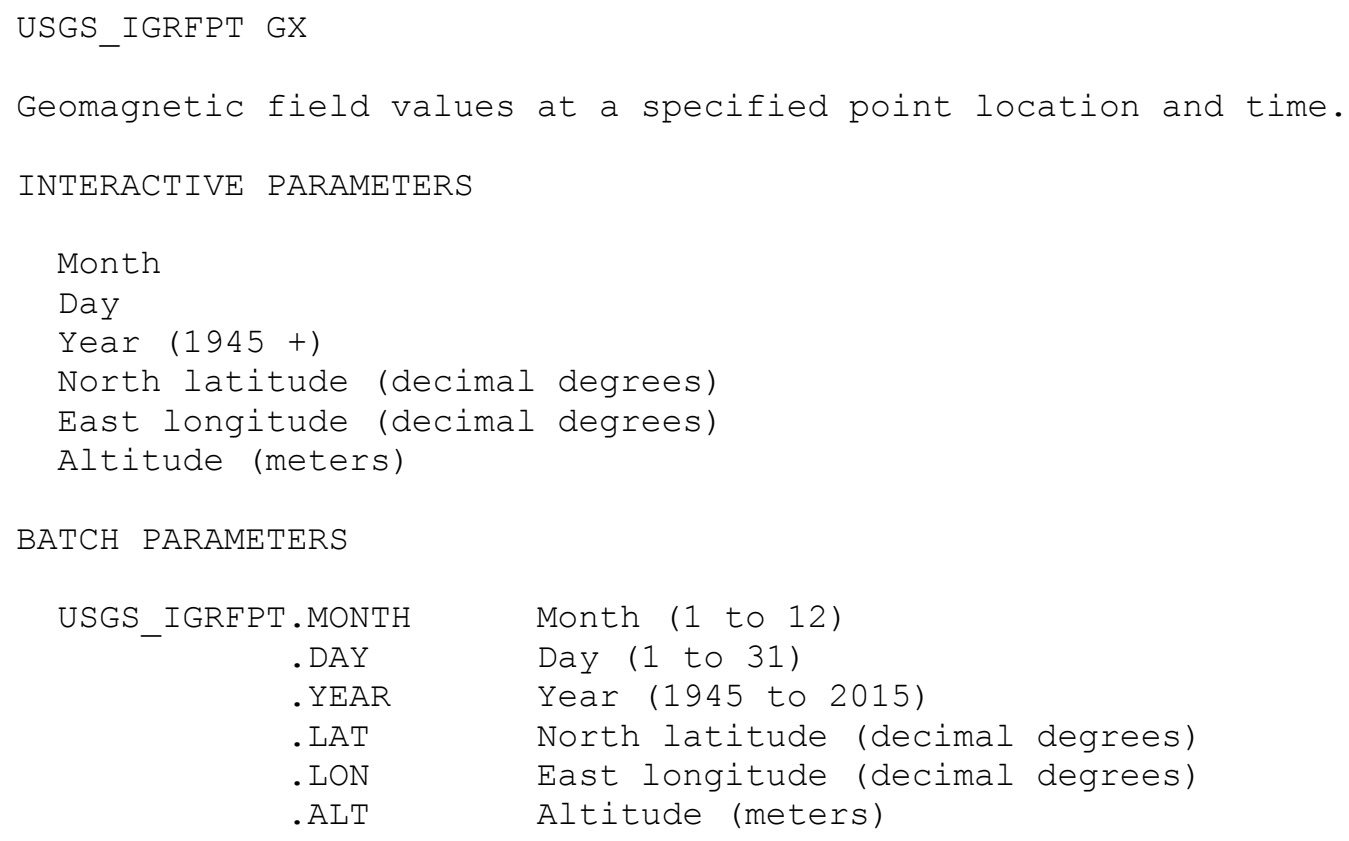




\section{USGS_IMPOST}

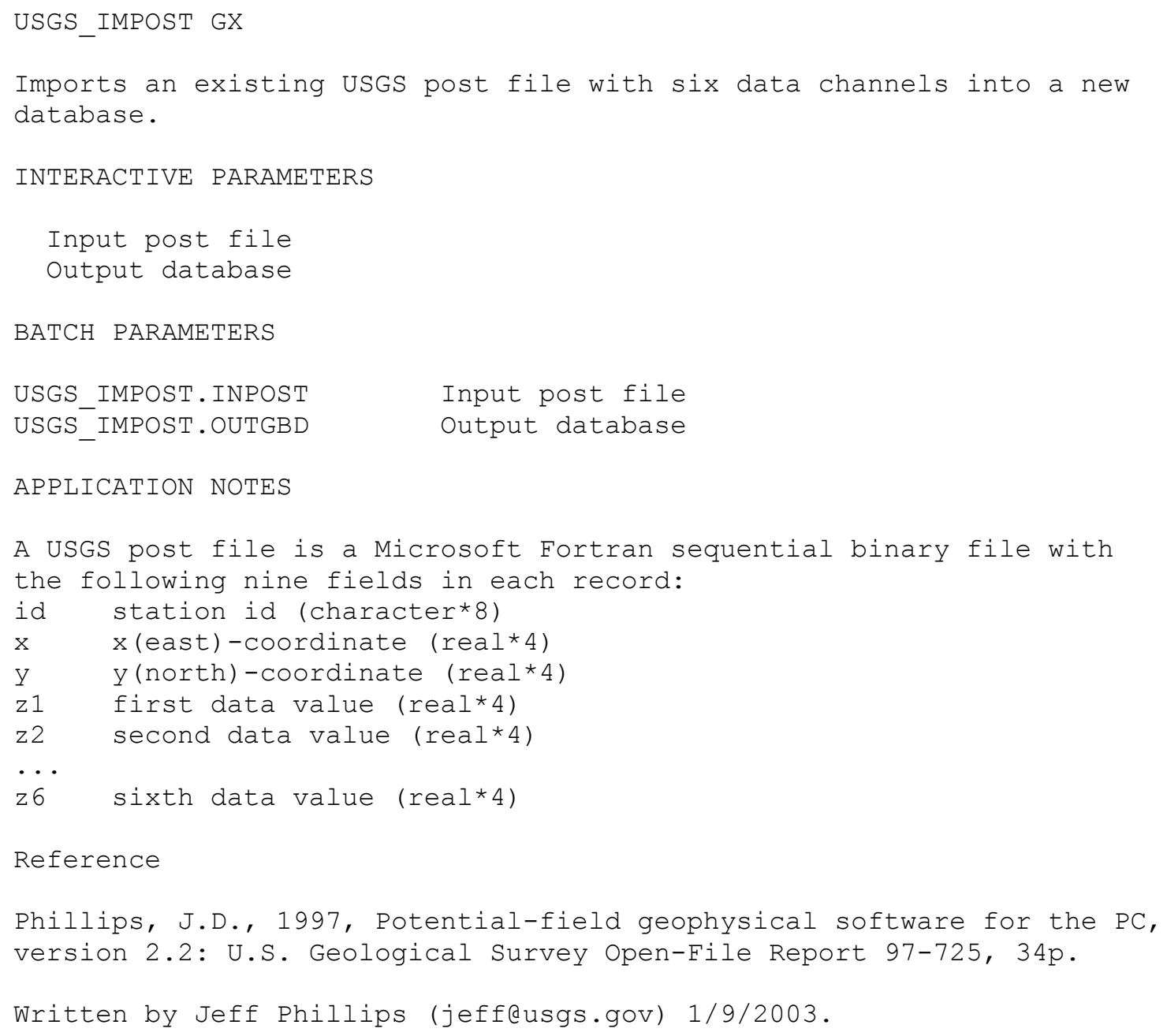




\section{USGS_LNPLOT}

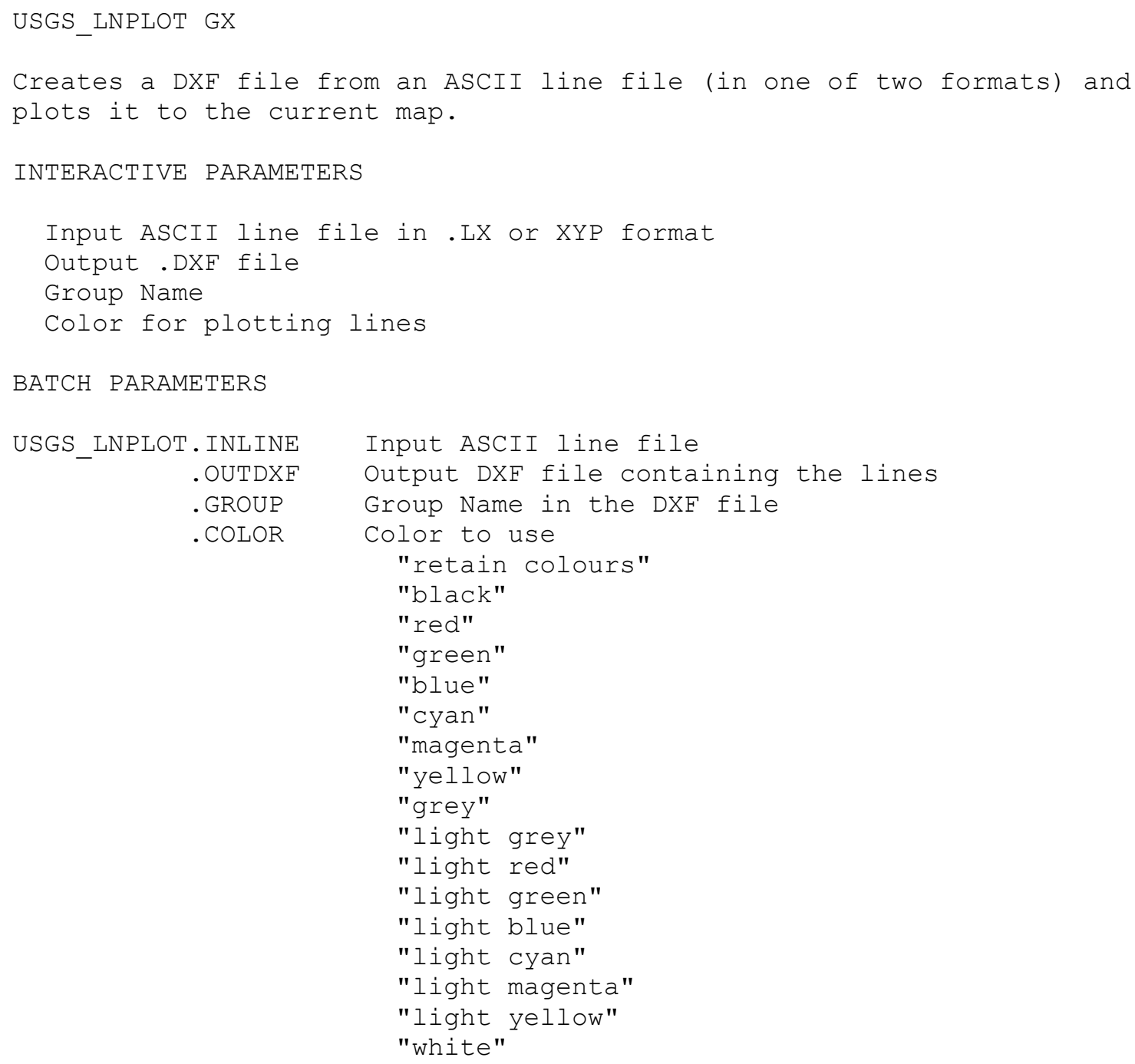

\section{APPLICATION NOTES}

An LX file such as myfile.lx is an ASCII file containing two columns separated by one or more spaces. The left column contains the $x$ (east)-coordinates of the polylines; the right column contains the $y$ (north)-coordinates. The end of each polyline is indicated by a value of $1 . e 38$ in each column.

An XYP file, which can have any extension other than. Ix, is an ASCII file containing three columns separated by one or more spaces. The left column contains the $x$ (east)-coordinates of the polylines, the center column contains the y(north)-coordinates, and the right column contains the polyline number. When the value in the right column changes, a new line is started.

The GX assumes that the $\mathrm{x}$ and $\mathrm{y}$ coordinates in the line file are in the same projection and distance units as the map. 
Written by Jeff Philips (jeff@usgs.gov) 03/03/2005. 


\section{USGS_LW}

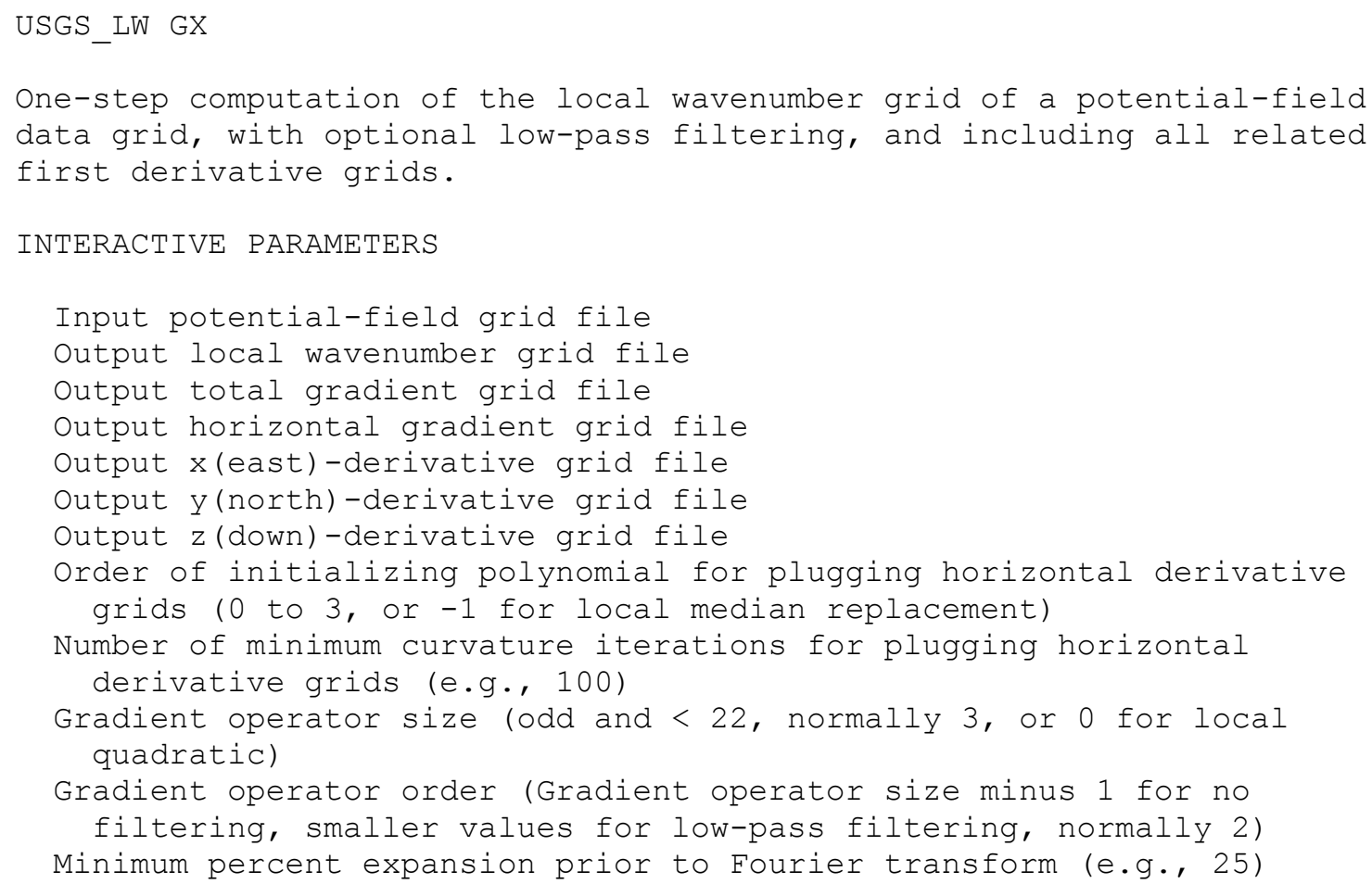

\section{APPLICATION NOTES}

The equation for the local wavenumber is given by Phillips (2000) and Phillips and others (2007).

If gradient operator size is 3 or greater, horizontal gradients are computed using differences (usgs_gradcomp.gx). If gradient operator order is less than gradient operātor size minus one, low-pass filtering will result (Thurston and Brown, 1994).

If gradient operator size is 0, horizontal gradients are computed using local quadratic surfaces (usgs_gradxyh.gx). Gradient operator order is ignored. 


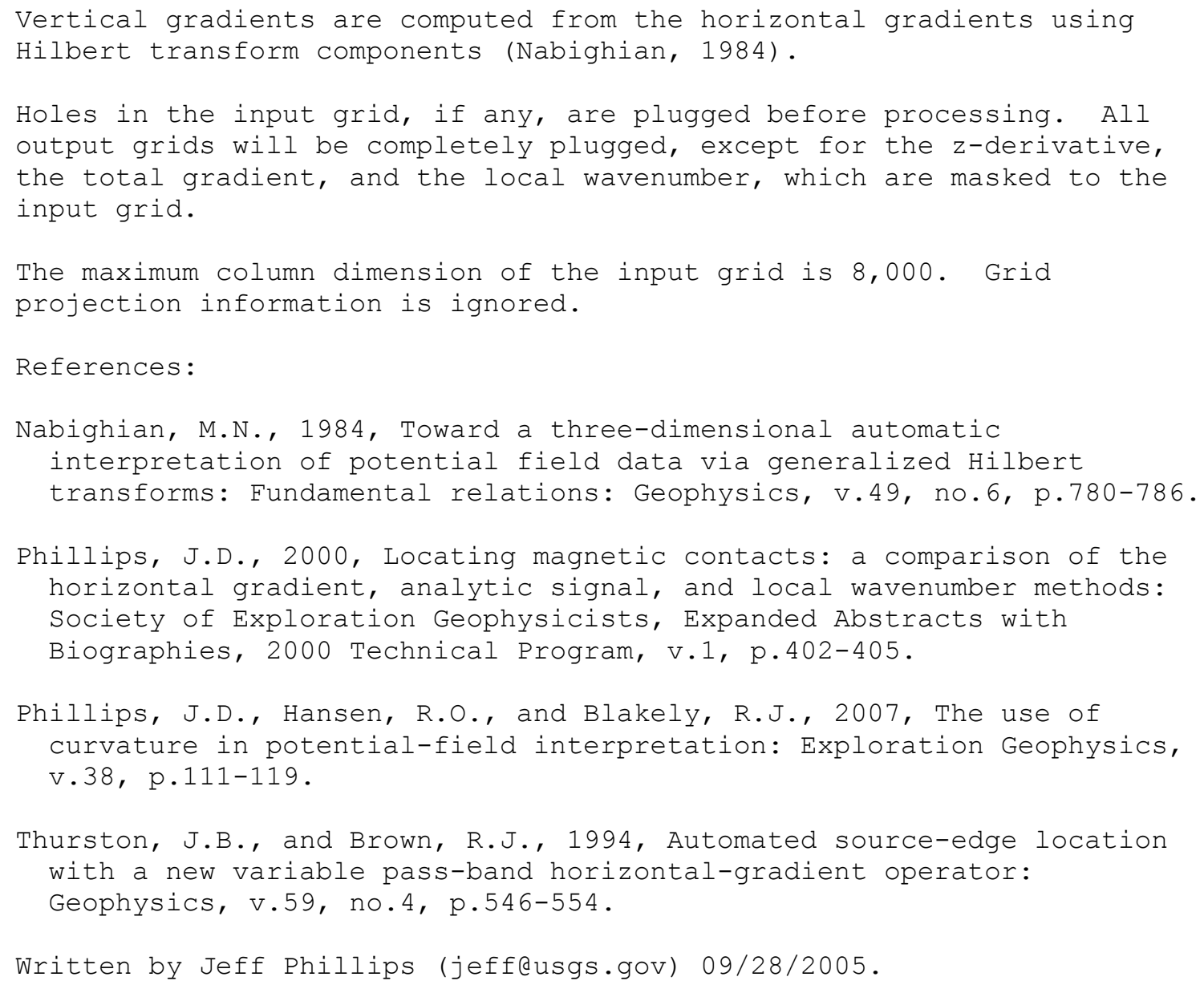




\section{USGS_MBASE}

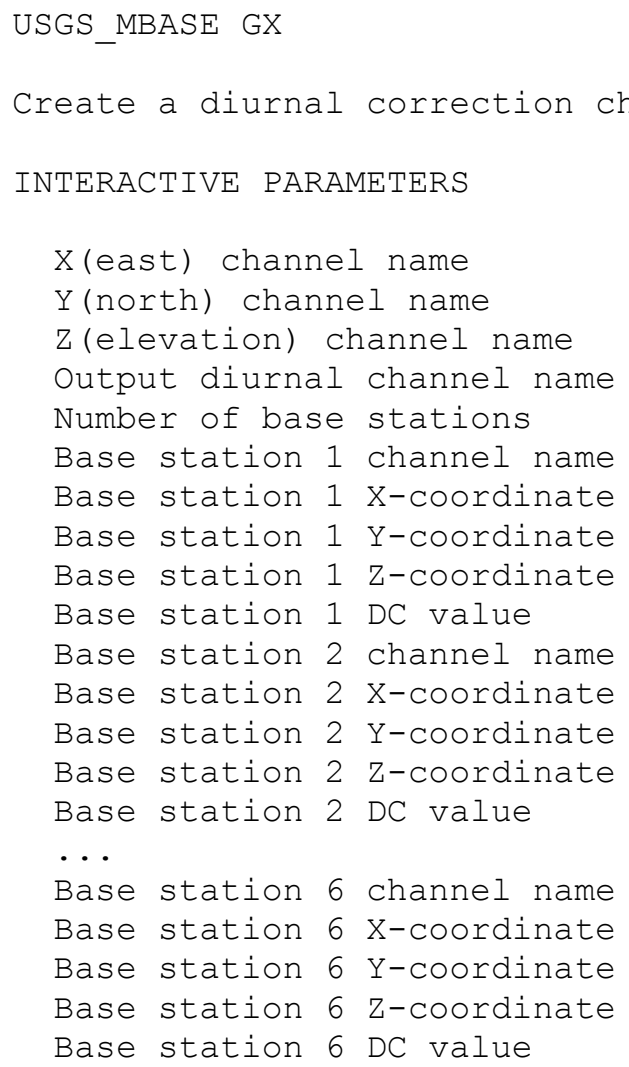

USGS_MBASE.XCHAN

USGS_MBASE.YCHAN

USGS ${ }^{-}$MBASE. ZCHAN

USGS ${ }^{-}$MBASE. OUTCHAN

USGS MBASE.NBASE

USGS-MBASE.B1CHAN

USGS_MBASE.B1X

USGS-MBASE.B1Y

USGS-MBASE.B1Z

USGS ${ }^{-}$MBASE . B1DC

USGS_MBASE.B2CHAN

USGS_MBASE.B2X

USGS_MBASE.B2Y

USGS MBASE.B2Z

USGS_MBASE.B2DC

$\ldots$

USGS_MBASE.B6CHAN

USGS-MBASE.B6X

USGS MBASE. B6Y

USGS-MBASE.B6Z

USGS_MBASE.B6DC
$X$ (east) channel name

$Y$ (north) channel name

Z (elevation) channel name

Output diurnal channel name

Number of base stations

Base station 1 channel name

Base station $1 \mathrm{X}$-coordinate

Base station 1 Y-coordinate

Base station 1 Z-coordinate

Base station 1 DC value

Base station 2 channel name

Base station 2 X-coordinate

Base station 2 Y-coordinate

Base station 2 Z-coordinate

Base station 2 DC value

Base station 6 channel name

Base station $6 \mathrm{x}$-coordinate

Base station 6 Y-coordinate

Base station 6 z-coordinate

Base station 6 DC value 
APPLICATION NOTES

For one base station, the base station value is returned.

For two base stations, the returned value is on the "most level" plane defined by the two base station values.

For three base stations, the returned value is on the plane defined by the three base station values.

For more than three base stations, the returned value is on a plane fitting all base station values, weighted by inverse distance.

Base station DC values are typically an average of quiet-time values

near local midnight. The averages should be performed over the same set of days for all base stations.

Written by Jeff Phillips (jeff@usgs.gov) 11/28/2006. 


\section{USGS_MFDESIGN}

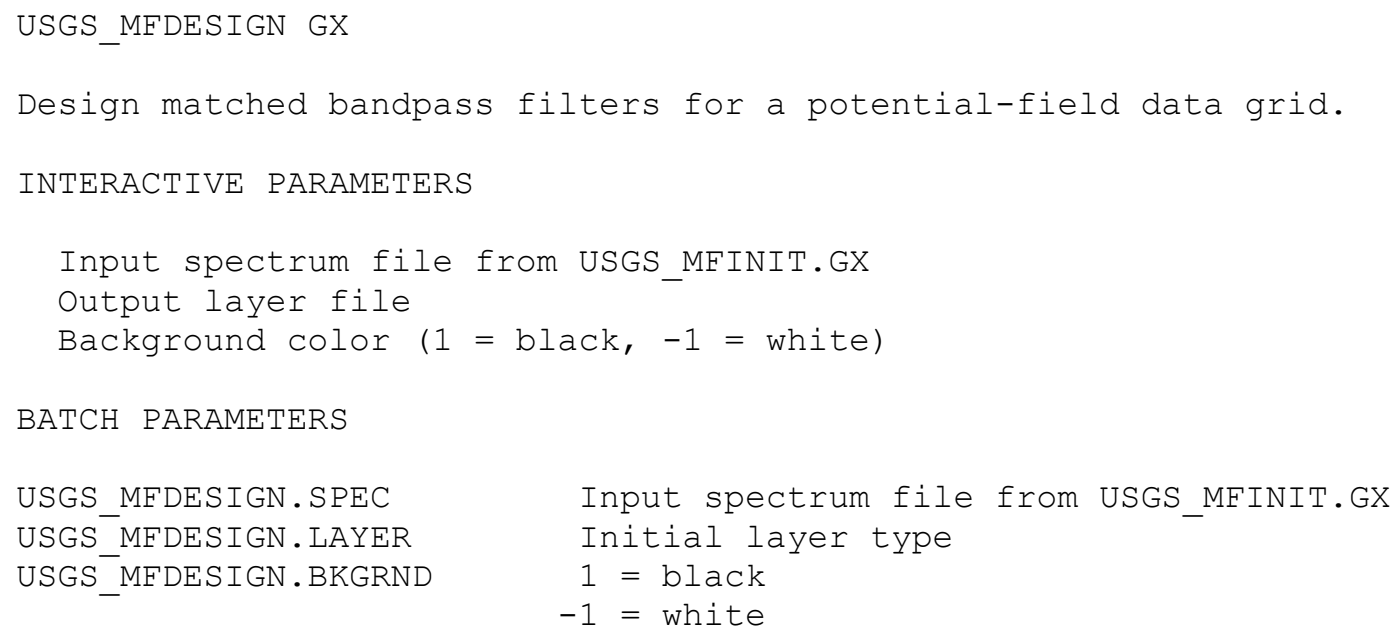

APPLICATION NOTES

This GX requires files generated by USGS_MFINIT.GX.

Start by fitting the the right-hand side of the plot with the shallowest equivalent layer, usually a dipole layer (magnetics) or a density layer (gravity). Then move to the left for each subsequent equivalent layer.

Use thin-layer models for the shallowest layers; switch to half-space models at the depth where you want the greatest spectral separation.

You can try different models for the current layer. Remember to move on to the next layer when you are happy with the fit, and select "all done" after fitting the deepest (left-most) layer.

The non-linear least squares iterative improvement is performed until the RMS error is minimized. This step is recommended.

Use USGS_MFFILTER.GX to apply the bandpass filters to the data.

Reference

Phillips, J.D., 2001, Designing matched bandpass and azimuthal filters for the separation of potential-field anomalies by source region and source type: Australian Society of Exploration Geophysicists, 15th Geophysical Conference and Exhibition, Expanded Abstracts CD-ROM, 4p.

Written by Jeff Phillips (jeff@usgs.gov) 2/10/2003. 


\section{USGS_MFFILTER}

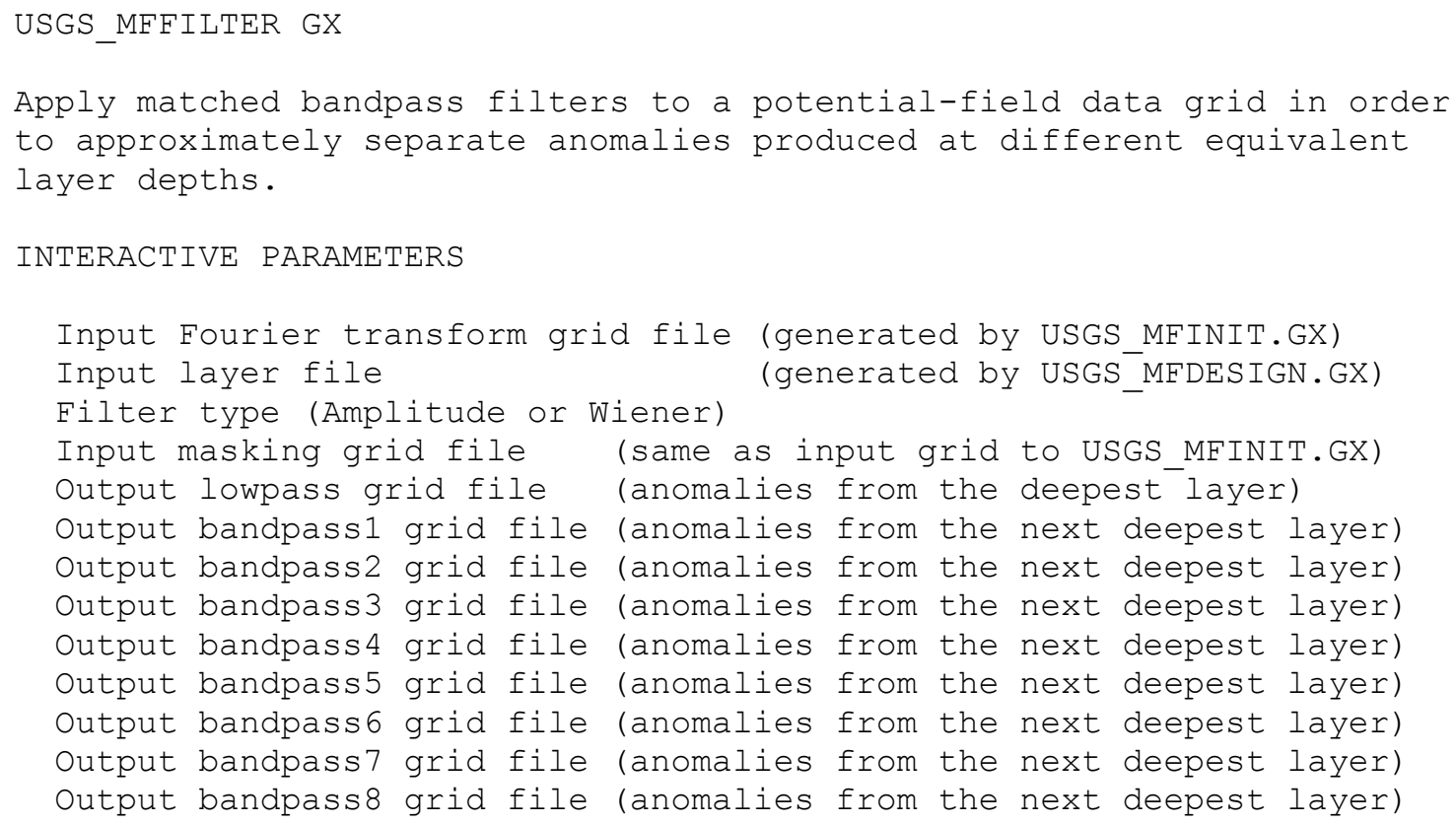

USGS_MFFILTER.INFFT

USGS_MFFILTER.INLAY

USGS MFFILTER. FTYPE USGS_MFEILTER.MASKGRD

USGS_MFFILTER.OUTGRDO USGS_MFEILTER.OUTGRD1

USGS_MFFILTER.OUTGRD2

USGS_MFEILTER.OUTGRD3

USGS_MFFILTER.OUTGRD4

USGS_MFFILTER.OUTGRD5

USGS_MFEILTER.OUTGRD6

USGS_MFFILTER.OUTGRD7

USGS_MFFILTER.OUTGRD8
Input Fourier transform grid file(from USGS_MFINIT.GX)

Input equivalent layer file (from USGS MFDESIGN.GX)

Filter type ("a" or "w")

Masking grid to be applied to the bandpass grids (the input grid to USGS MFINIT.GX)

Output lowpass grid file (deepest layer)

Output bandpassl grid file (the next deepest layer)

Output bandpass2 grid file (the next deepest layer)

Output bandpass3 grid file (the next deepest layer)

Output bandpass4 grid file (the next deepest layer)

Output bandpass5 grid file (the next deepest layer)

Output bandpass6 grid file (the next deepest layer)

Output bandpass7 grid file (the next deepest layer)

Output bandpass 8 grid file (the next deepest layer)

APPLICATION NOTES

Requires files generated by USGS_MFINIT.GX and USGS_MFDESIGN.GX. 
You only need to specify as many output grids as you have layers in the layer file.

The maximum column dimension of the masking and output grids is 8,000. Grid projection information is ignored.

\section{Reference}

Phillips, J.D., 2001, Designing matched bandpass and azimuthal filters for the separation of potential-field anomalies by source region and source type: Australian Society of Exploration Geophysicists, 15th Geophysical Conference and Exhibition, Expanded Abstracts CD-ROM, 4p. Written by Jeff Phillips (jeff@usgs.gov) 2/10/2003. 


\section{USGS_MFPLOT}

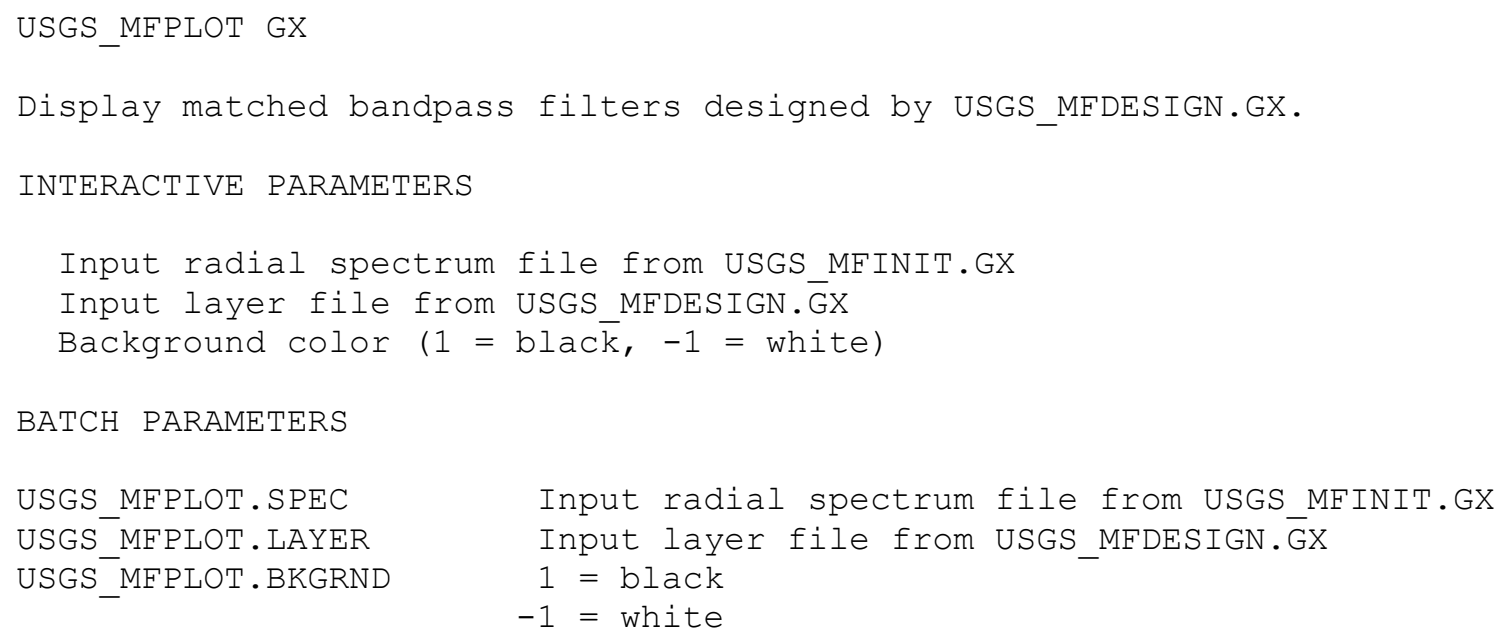




\section{USGS_OPENPOST}

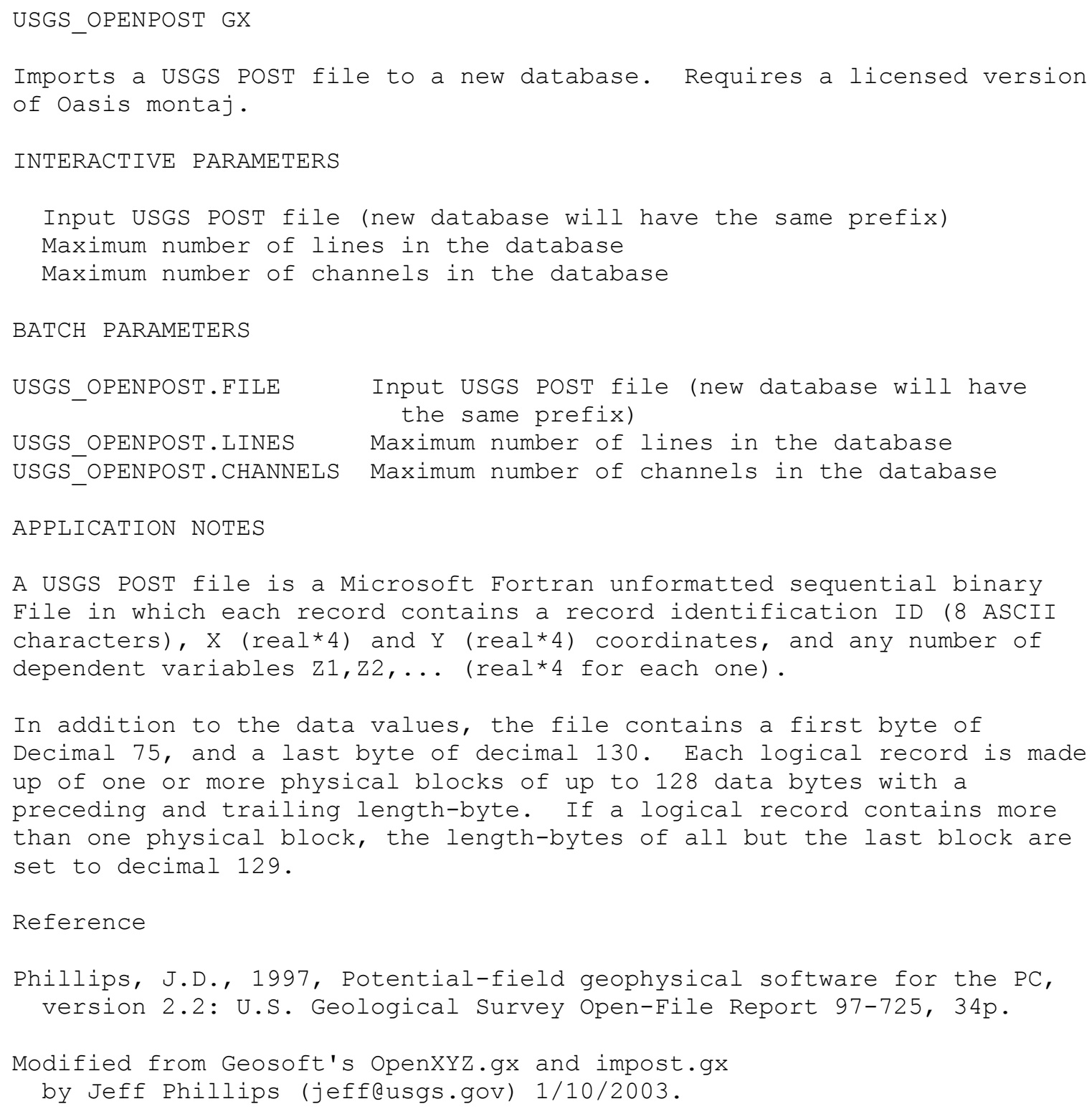




\section{USGS_PDRAW}

USGS PDRAW GX

Draws the polygons from a polygon file into a specified group on the current map.

INTERACTIVE PARAMETERS

Polygon file name (.ply)

Map view

Line thickness (mm)

Line colour

Fill colour

Group Name

Group Action

BATCH PARAMETERS

USGS_PDRAW. FILE
USGS_PDRAW. VIEW
USGS_PDRAW. LINETHICK
USGS_PDRAW. LINECOLO
USGS_PDRAW. FILLCOLO
USGS_PDRAW. GROUPNAME
USGS_PDRAW. GROUPACTN

The name of the polygon file

Map view in which to draw, select from the list

The polygon outline line thickness

in $\mathrm{mm}$

Enter a line colour of form

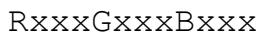

Enter a fill colour of form $\mathrm{R} x \mathrm{x} \times \mathrm{G} x \mathrm{x} \times \mathrm{B} \times \mathrm{x} x$

Enter the group name

Enter a zero (0) to replace the group (i.e. erase and replot) and a one (1) to append polygons to the group

Polygon file

Map view

Line thickness

Line color

Fill color

Group name

Group action

\section{APPLICATION NOTES}

Polygons are ASCII files with default extension.ply. A polygon file contains a list of $X, Y$ coordinates that define one or more polygons. The file may contain any number of polygons, and each polygon may have any number of vertices. The first and last points in each polygon are assumed to connect. If the file will contain more than one polygon, each polygon must start with a line 'poly \#' ('p' or ' $\mathrm{P}$ ' in column 1). Comment lines are indicated by a '/' in column 1. Please note that the polygon coordinates are assumed to be in the same coordinate system as that of the map view on which the polygons will be drawn.

Following is an example of a single polygon file:

/

/ Sample single polygon file 


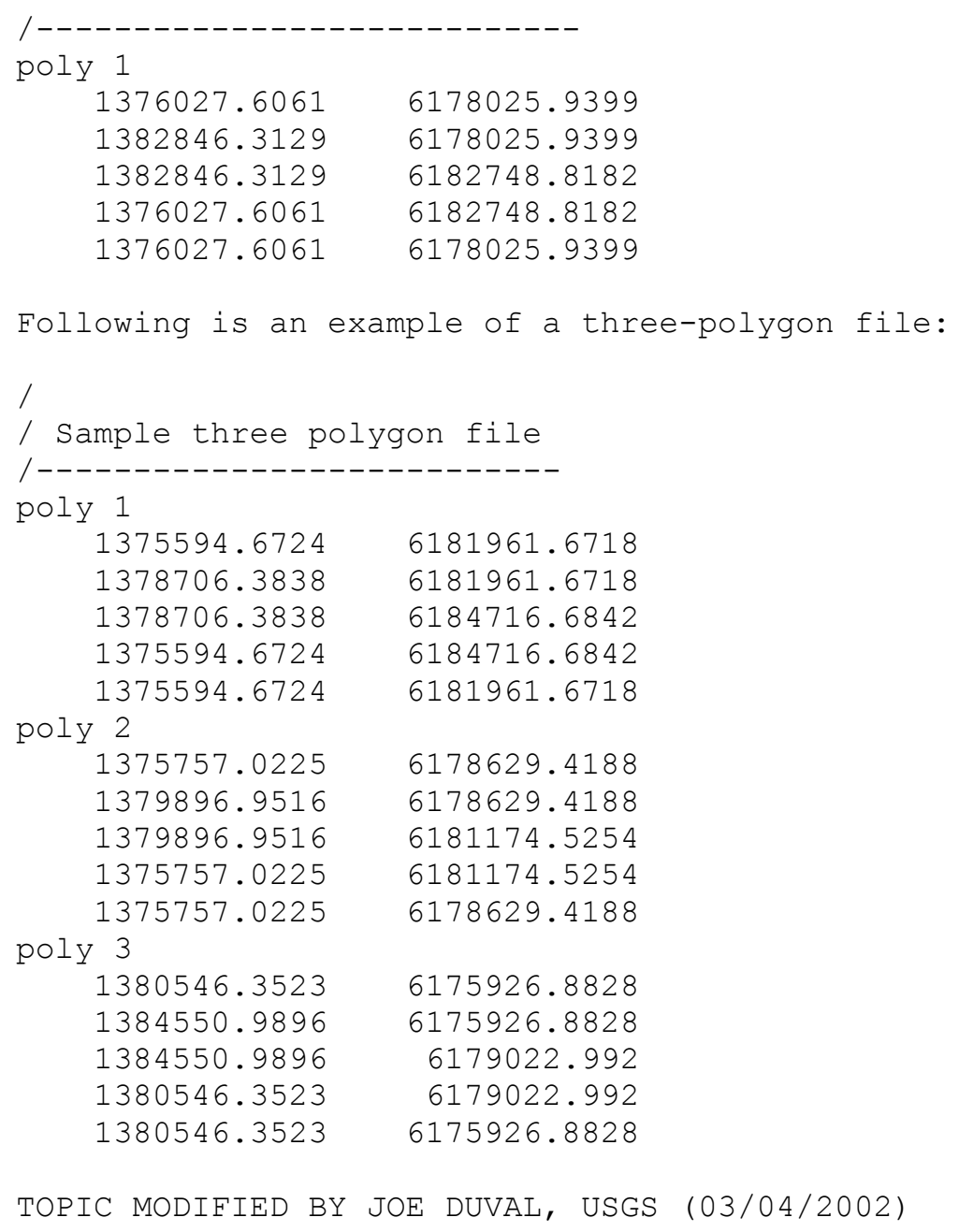




\section{USGS_PGPLOT}

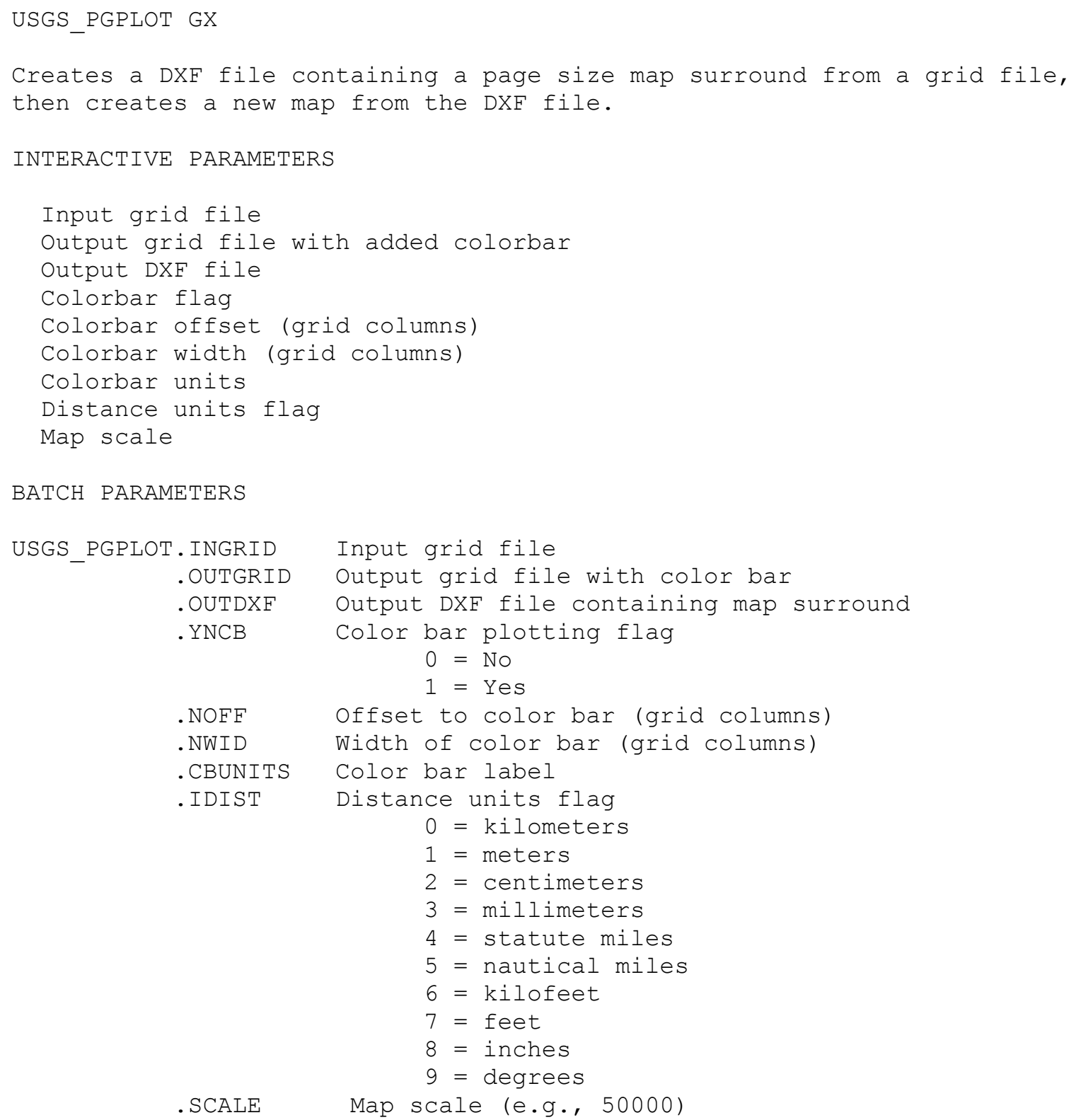


with shading.

Individual components of the base map can be displayed or hidden using the Group Manager.

Written by Jeff Philips (jeff@usgs.gov) 03/02/2005. 


\section{USGS_PLUGGRID}

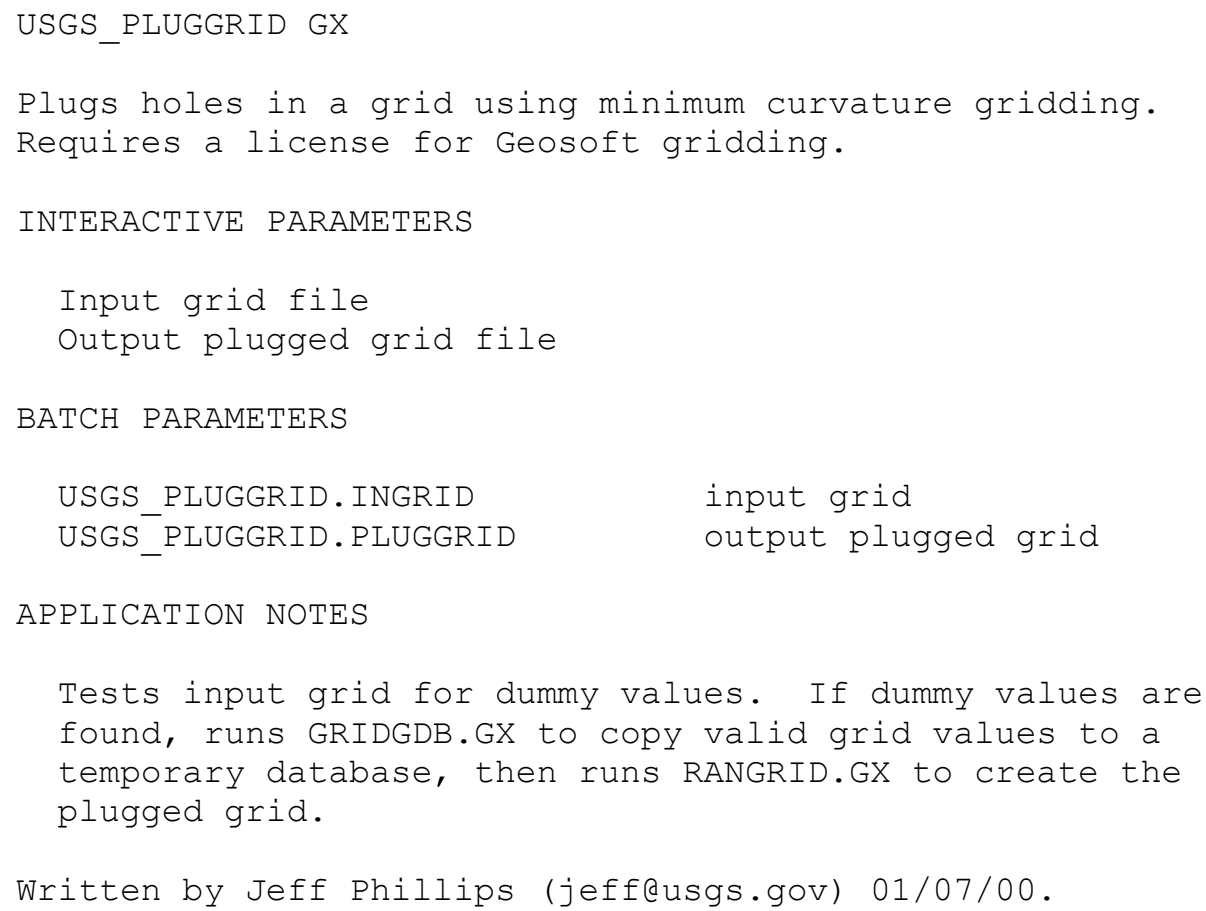




\section{USGS_PREP4}

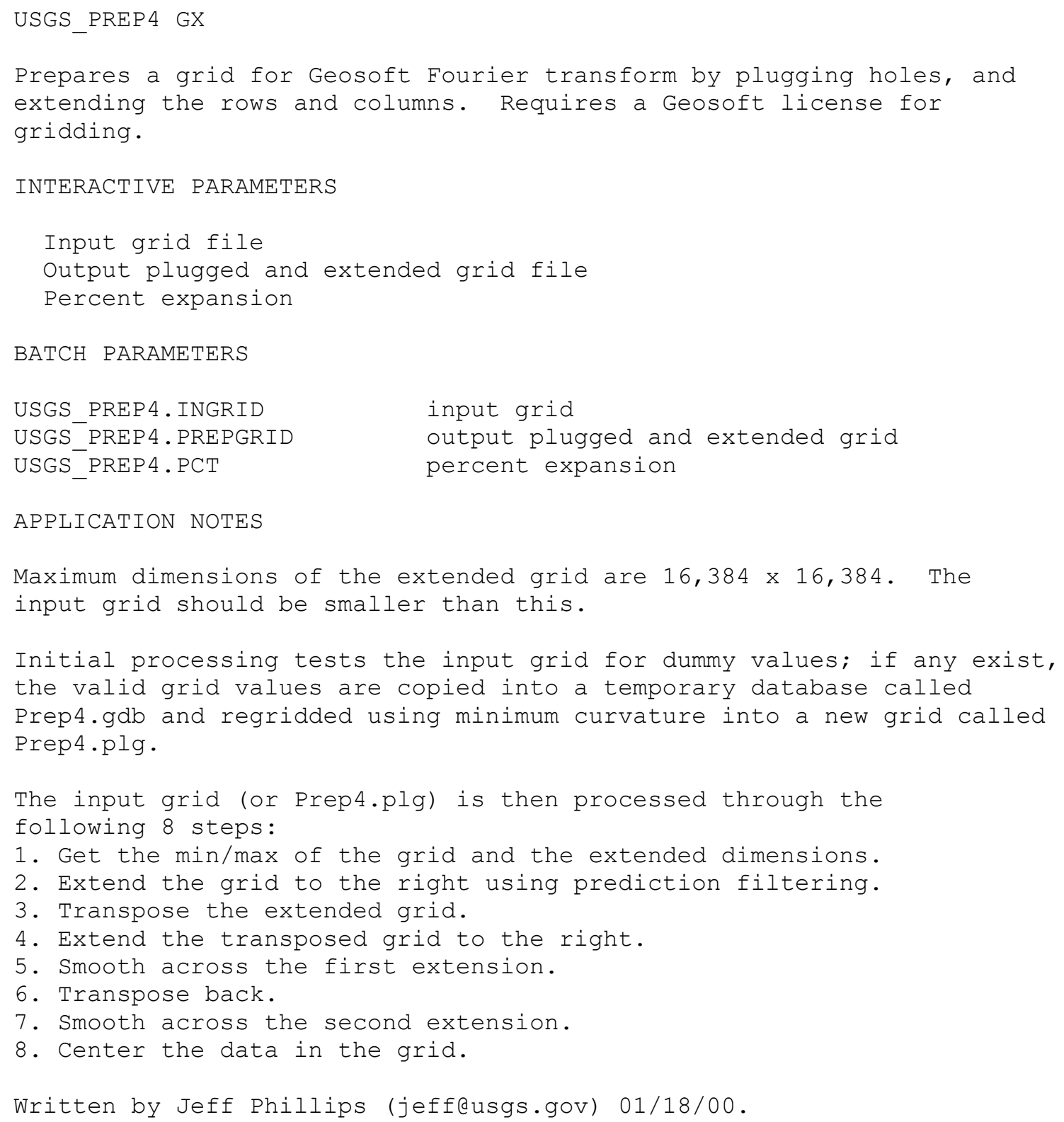




\section{USGS_PSDGRV}

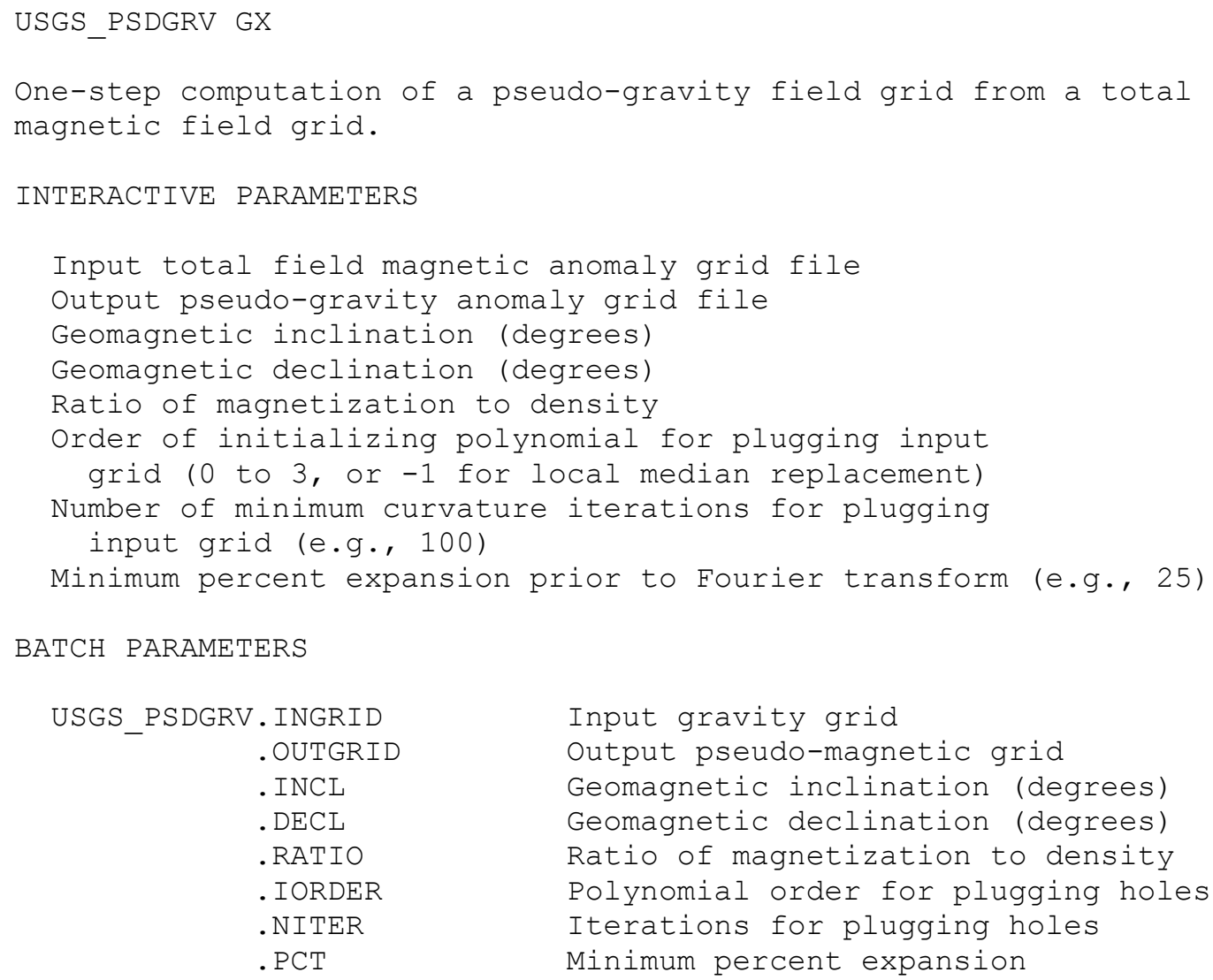

APPLICATION NOTES

Like standard reduction-to-the-pole, this will not work well at low magnetic latitudes (within 20 degrees of the magnetic equator). The maximum number of columns in the expanded input grid is 16,384. Grid projection information is ignored.

Written by Jeff Phillips (jeff@usgs.gov) 06/10/2003. 


\section{USGS_PSDMAG}

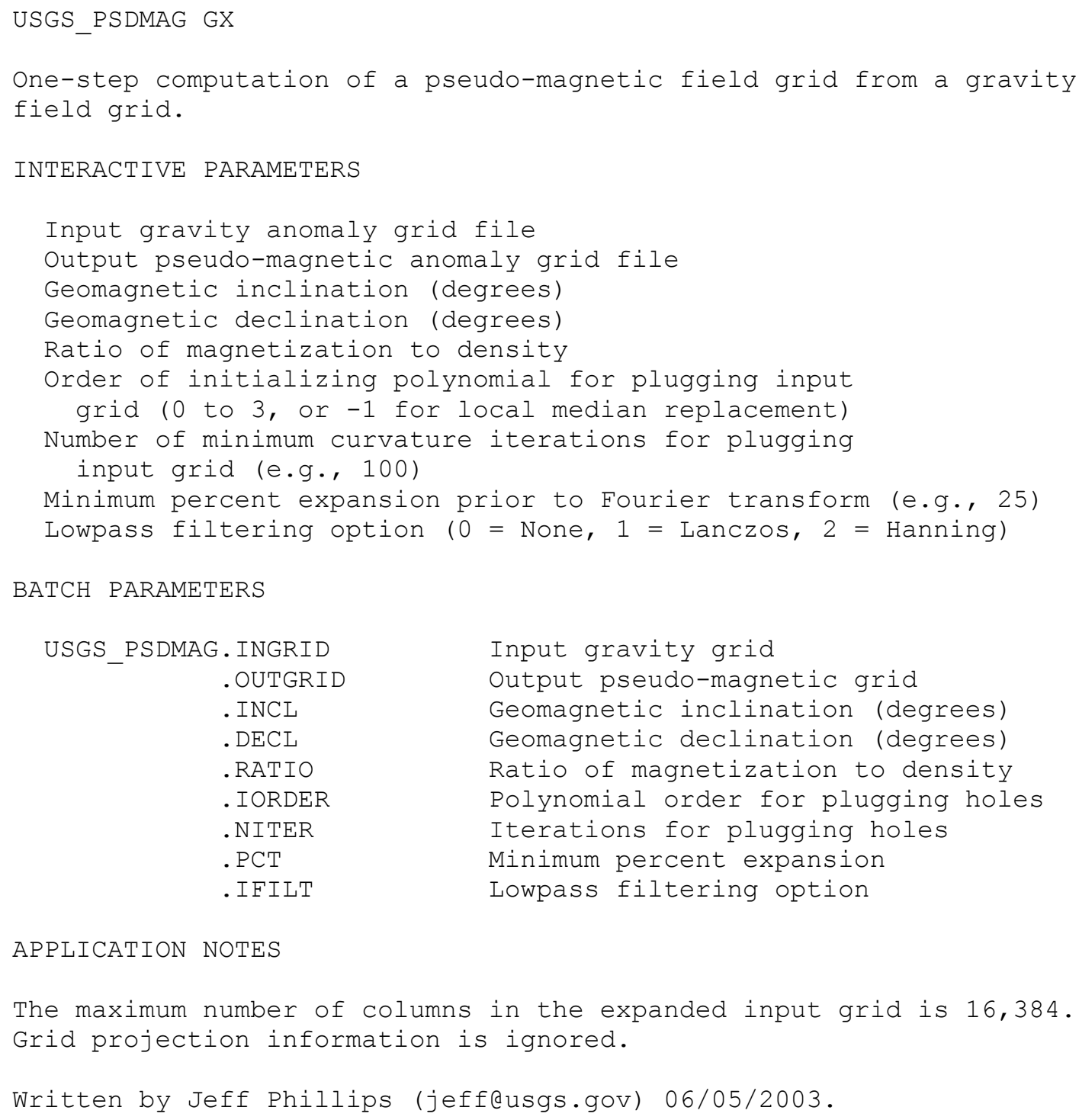

APPLICATION NOTES

The maximum number of columns in the expanded input grid is 16,384. Grid projection information is ignored.

Written by Jeff Phillips (jeff@usgs.gov) 06/05/2003. 


\section{USGS_RCDEP}

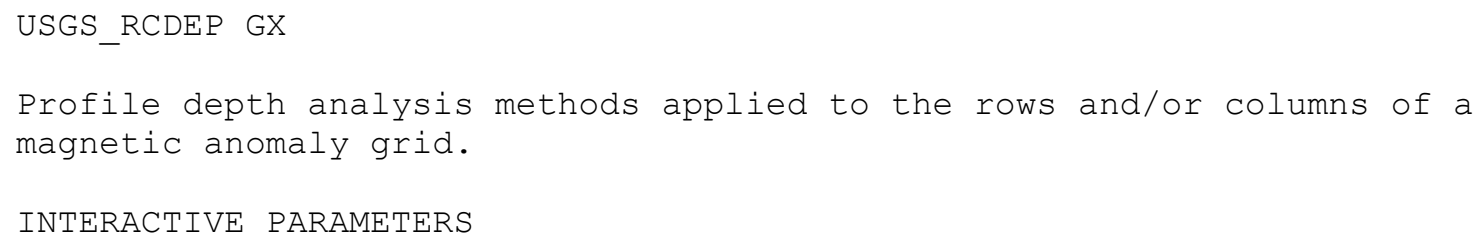

BATCH PARAMETERS

USGS_RCDEP.AGRID

. IORDER

. NITER

. OSGRID

. CONV

.TYPE

.STYP

. SDIR

- AMP

. IPEAK

. OUTPOST
Input magnetic anomaly grid (must be reduced-to-pole magnetic field for horizontal gradient contacts or pseudogravity for horizontal gradient sheets)

Polynomial order for plugging

Iterations for plugging

Optional input observation surface grid

Elevation units per horizontal units

Method used for depth analysis: 1=analytic signal, 2=horizontal gradient, or $3=$ local wavenumber

Desired source type for analytic signal or horizontal gradient analysis: $0=$ contact or 1 =sheet

Desired strike direction of features: 1 =north, 2 =east, or 3 =both

Minimum amplitude of the derivative function

Output closed peaks only: $0=$ No, $1=$ Yes

Output post file (and database)

\section{APPLICATION NOTES}

If an observation surface grid is used, it must have the same origin, number of rows and columns, and sample interval as the magnetic anomaly grid. The maximum number of columns in the input grids is 8,000. 
Reference

Phillips, J.D., 2000, Locating magnetic contacts: a comparison of the horizontal gradient, analytic signal, and local wavenumber methods: Society of Exploration Geophysicists, Expanded Abstracts with Biographies, 2000 Technical Program, v.1, p.402-405

Written by Jeff Phillips (jeff@usgs.gov) 04/15/2003. 


\section{USGS_REDPOL}

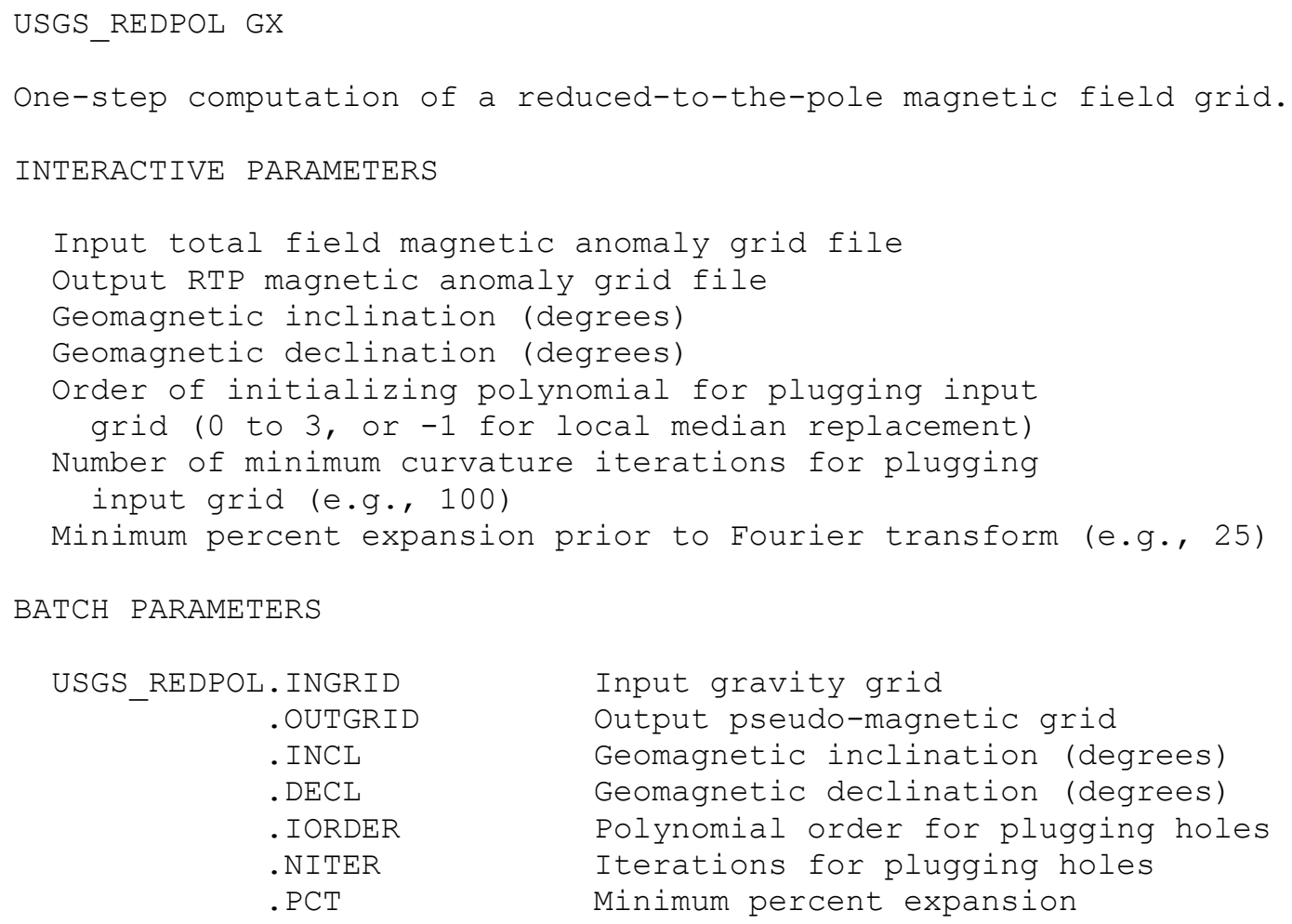

APPLICATION NOTES

This standard reduction-to-the-pole filter will not work well at low magnetic latitudes (within 20 degrees of the magnetic equator). The maximum number of columns in the expanded input grid is 16,384. Grid projection information is ignored.

Written by Jeff Phillips (jeff@usgs.gov) 06/10/2003. 


\title{
USGS_RENUMLNS
}

\author{
USGS_RENUMLNS GX
}

Renumber all selected lines. This is useful for changing line numbers prior to merging databases.

INTERACTIVE PARAMETERS

The starting line number.

The line number increment for numbering sequential lines.

BATCH PARAMETERS

$\begin{array}{ll}\text { USGS_RENUMLNS.STRTLN } & \text { Starting line number } \\ \text { USGS_RENUMLNS.INCRLN } & \text { Line number increment }\end{array}$

APPLICATION NOTES

If any parameters are blank, the default parameter value is set to 1 . The process will stop if the new name of a selected line already exists. All lines processed up to that point will be changed.

TOPIC WRITTEN BY JOE DUVAL, USGS (11/21/2000) 


\section{USGS_RTFIL}

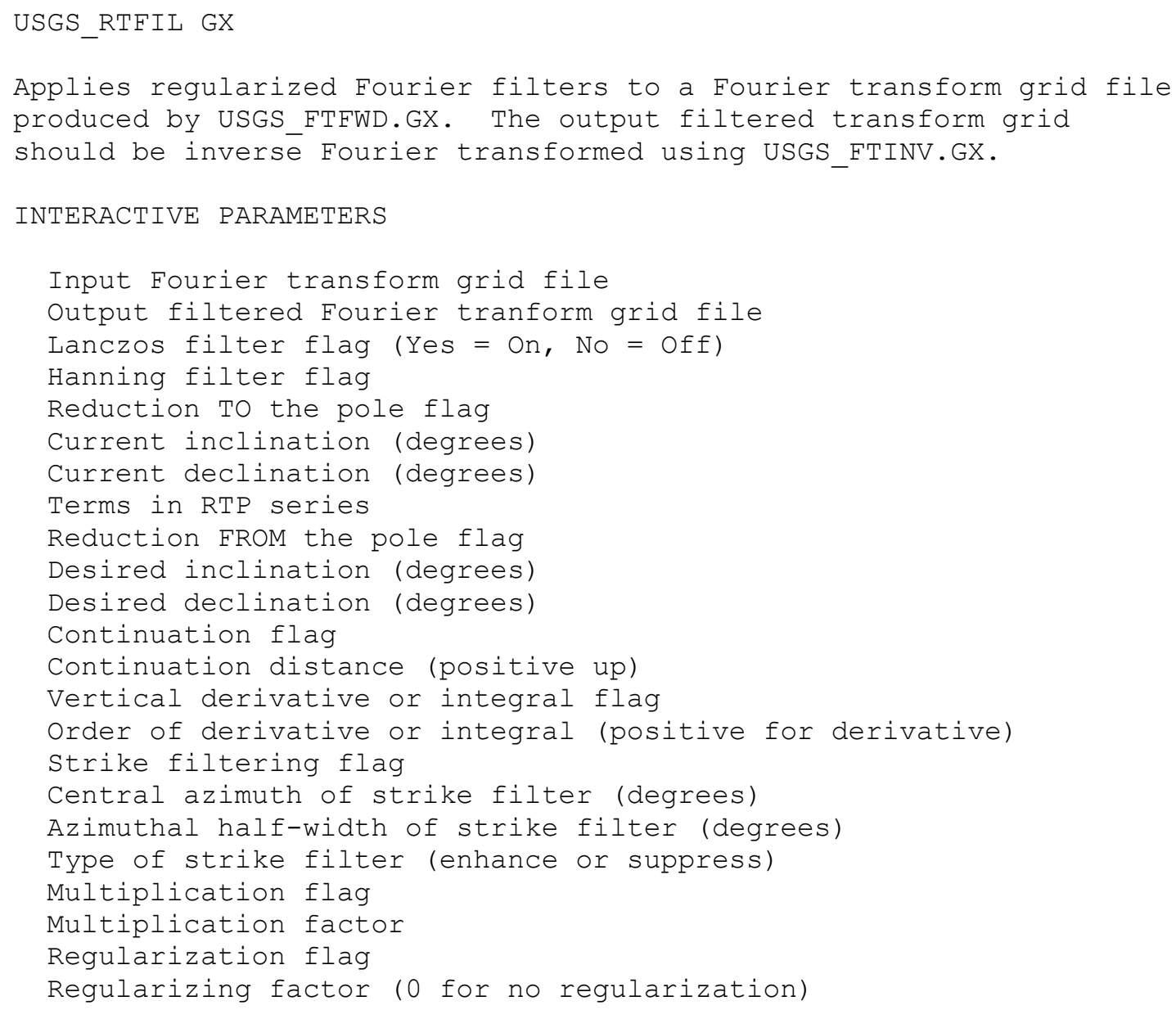

USGS RTFIL.INFFT

USGS_RTFIL.OUTFFT

USGS RTFIL. ILANC

USGS RTFIL. IHANN

USGS ${ }^{-}$RTFIL. IPOLE

USGS RTFIL. AINCL

USGS RTFIL.ADECL

USGS_RTEIL.NPTS

USGS RTFIL.IFROM

USGS RTFIL.BINCL

USGS RTFIL.BDECL

USGS RTFIL. ICONT

USGS RTFIL.HEIGHT

USGS RTFIL.IVERT

USGS_RTFIL.IORDER

USGS RTFIL.ISTRK

USGS_RTFIL.ASTRK

USGS_RTEIL.WSTRK

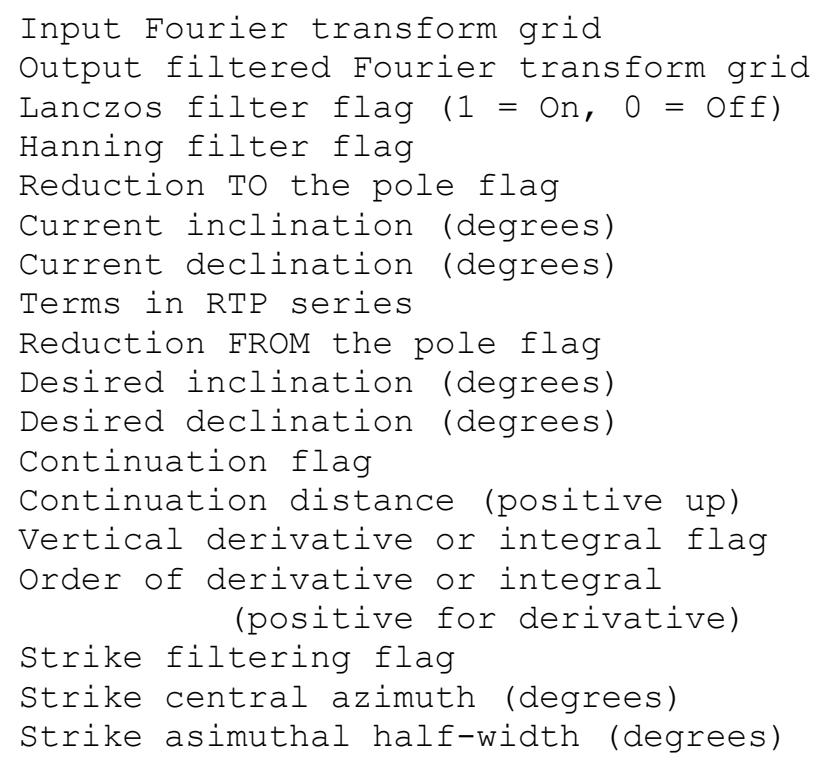




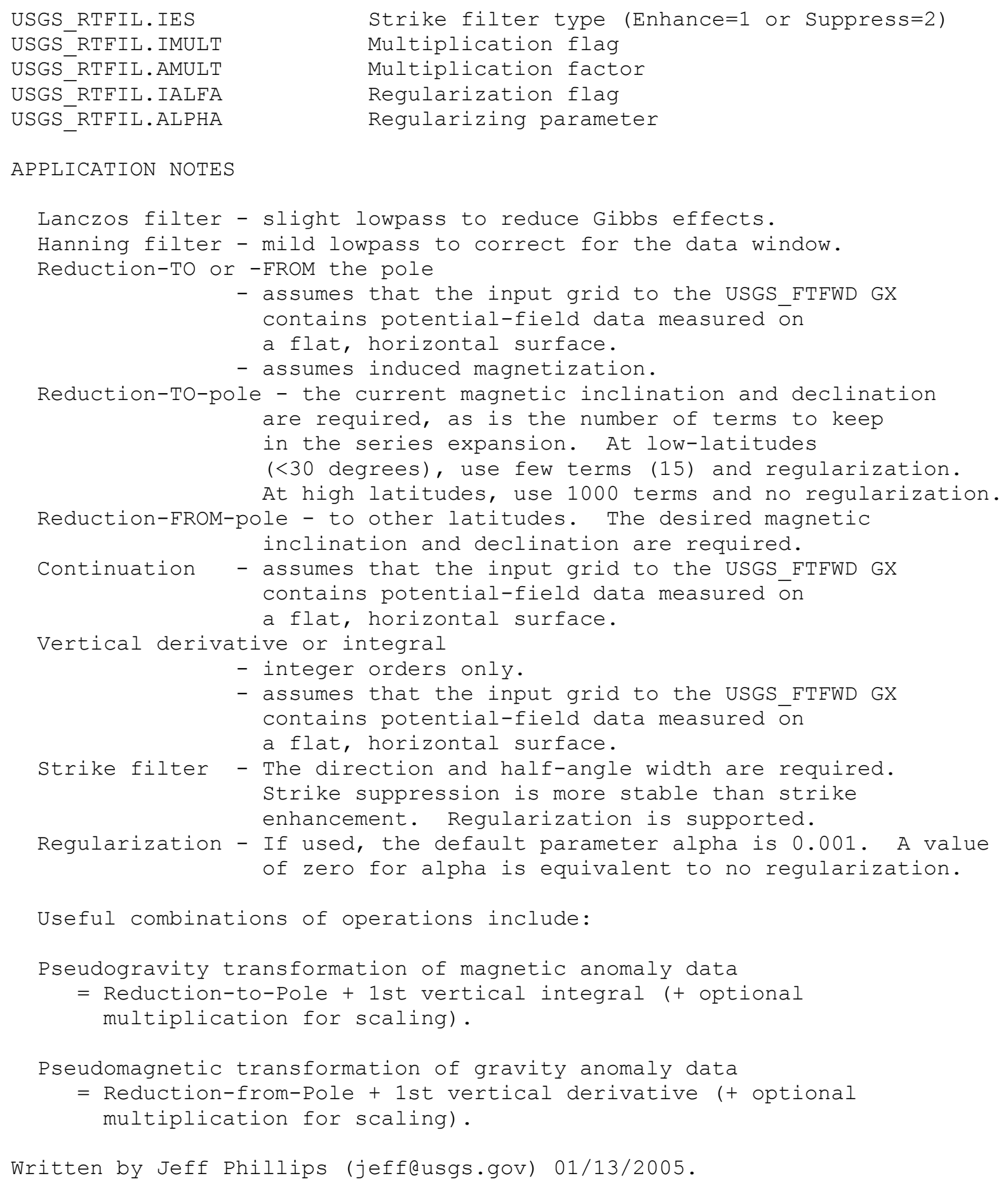

APPLICATION NOTES 


\section{USGS_SETEULER}

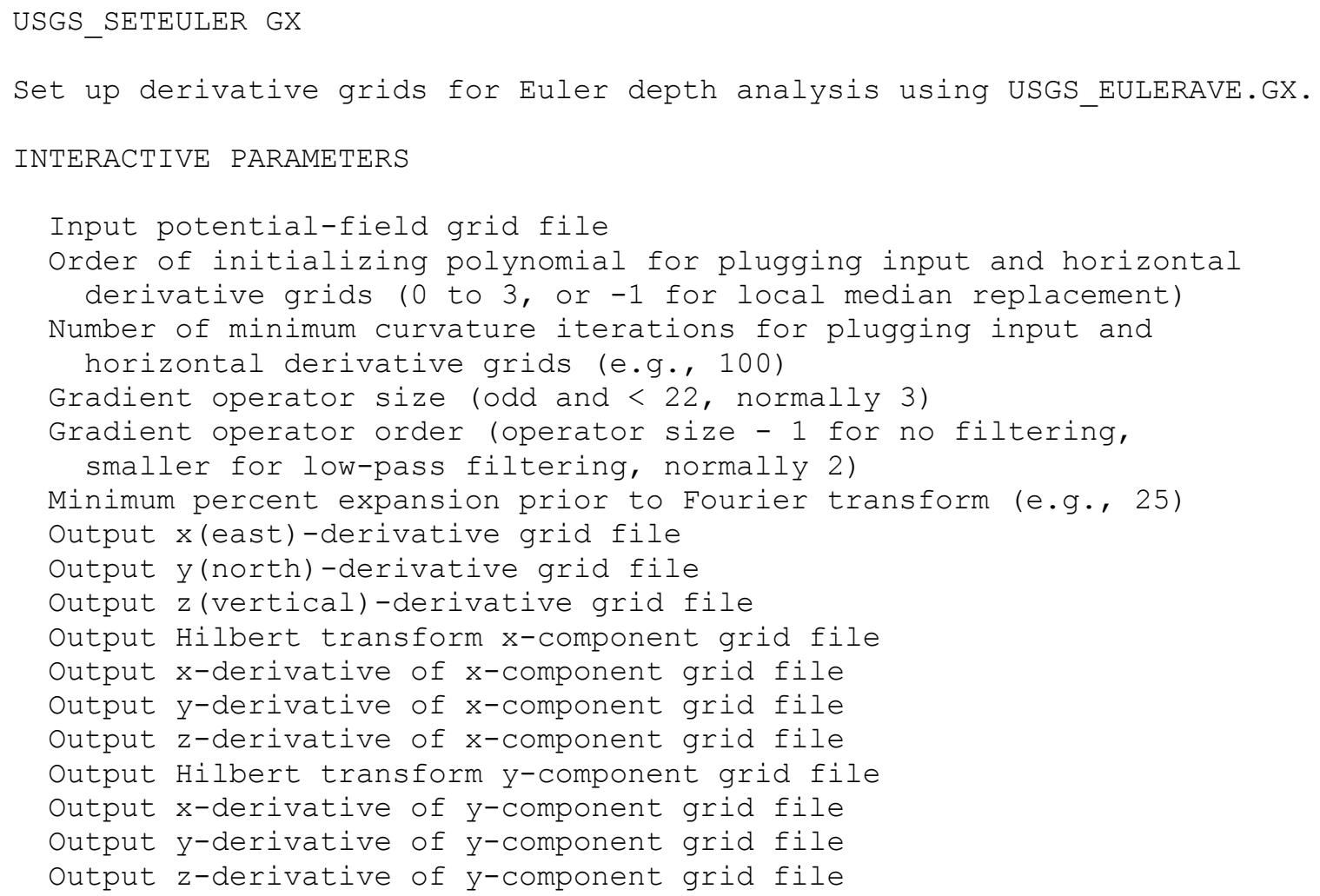

\section{APPLICATION NOTES}

The maximum number of columns in the input grid is 8,000. Grid projection information is ignored.

Written by Jeff Phillips (jeff@usgs.gov) 04/07/2003. 


\title{
USGS_SFDEPTH
}

\author{
USGS_SFDEPTH GX \\ Estimates potential field source locations from a special function grid. \\ Model-specific special function grids require that a specific source \\ type be assumed and the field be transformed before the special function \\ is calculated. Model-specific special functions include the Horizontal \\ Gradient Magnitude (HGM) and the Absolute Value (ABS) of the transformed \\ field. The possible transformations include Reduction-to-Pole (RTP), \\ Reduction-to-Pole plus Vertical Integral (RTP+VI), Vertical Derivative \\ (VD), or None. The following model-specific special functions are \\ Supported (SI is structural index):
}

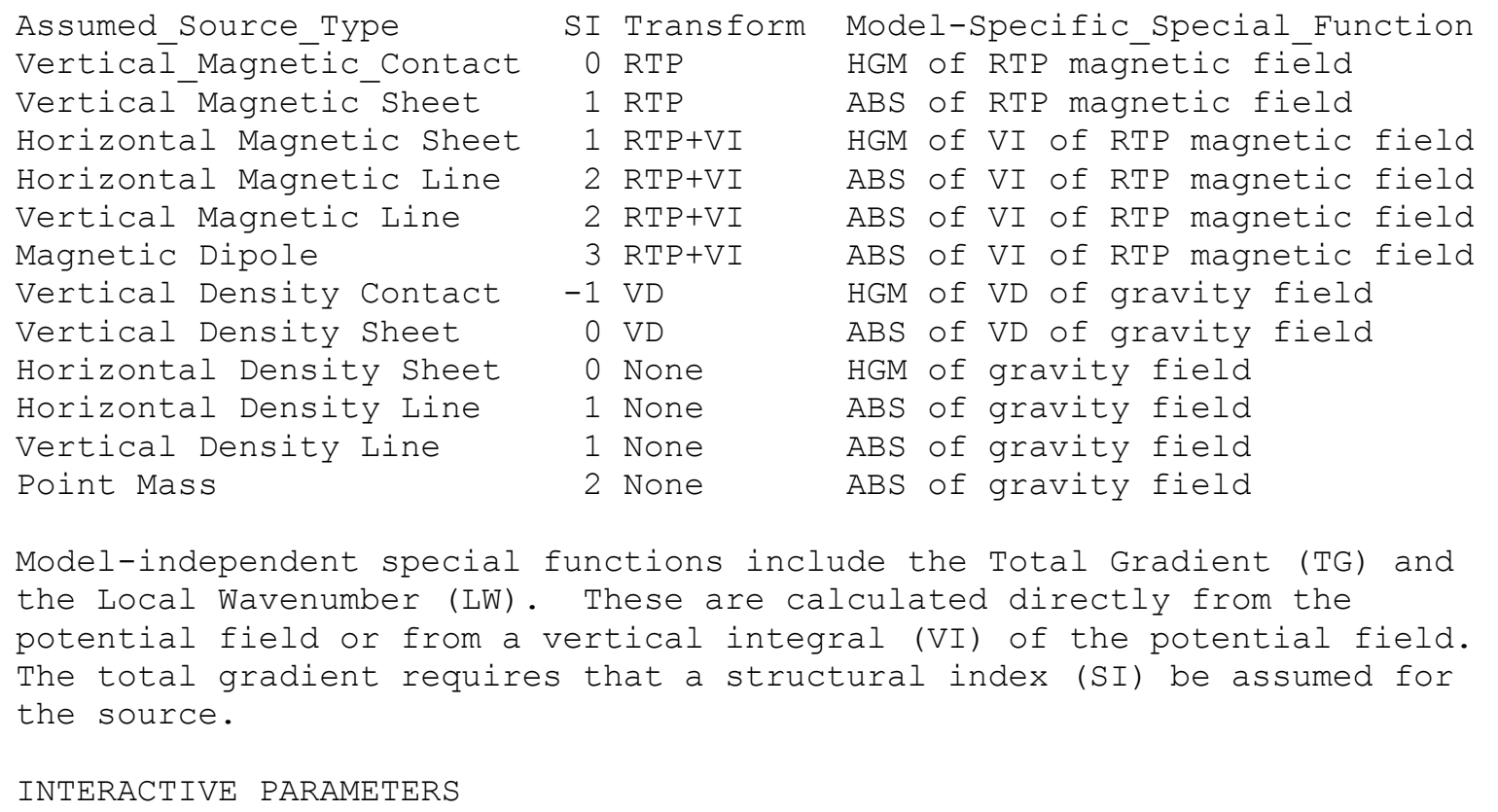

Input special function grid file

Output post file (and database)

Minimum special function amplitude to use in the analysis (normally zero)

Type of special function (ABS, HGM, TG, LW)

Type of feature to locate (Ridge, High, Both)

Assumed source type for ABS analysis

Assumed source type for HGM analysis

Assumed structural index for TG analysis

Order of vertical integral used for TG

Minimum structural index for $L W$ analysis

Maximum structural index for $L W$ analysis

Flag to adjust or discard LW solutions outside SI range

Order of vertical integral used for $L W$

BATCH PARAMETERS 


USGS_SFDEPTH.INGRID
USGS_SFDEPTH. OUTPST
USGS_SFDEPTH. AMPMIN
USGS_SFDEPTH.TYP
USGS_SFDEPTH.FTYP
USGS_SFDEPTH.ATYP
USGS_SFDEPTH.HTYP
USGS_SFDEPTH.TSI
USGS_SFDEPTH.TVI
USGS_SFDEPTH.SIMIN
USGS_SFDEPTH.SIMAX
USGS_SFDEPTH.ICOR
USGS_SFDEPTH.LVI

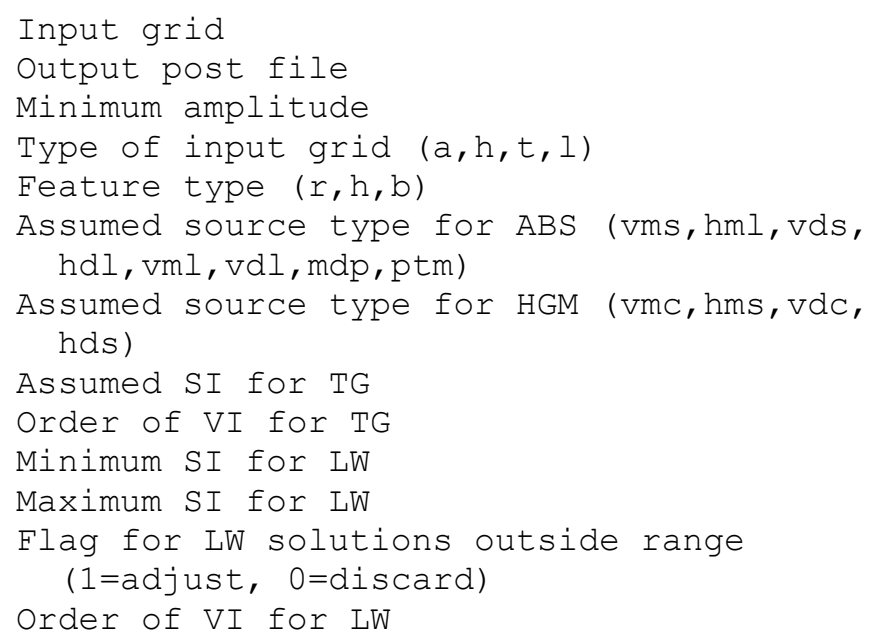

APPLICATION NOTES

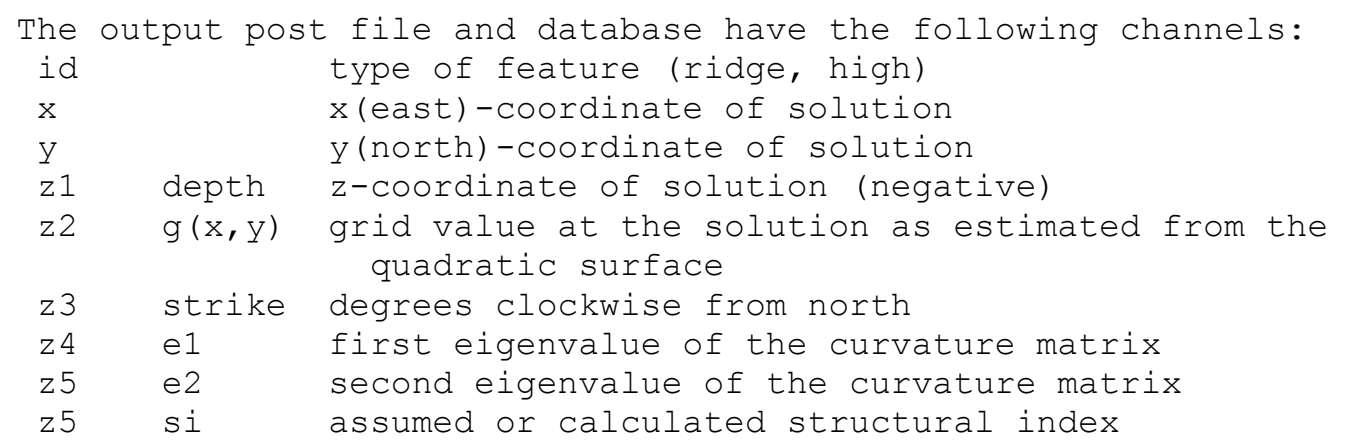

The maximum number of columns on the input grid is 8,000.

Reference

Phillips, J.D., Hansen, R.O., and Blakely, R.J., 2007, The use of curvature in potential-field interpretation: Exploration Geophysics, v.38, p.111-119.

Written by Jeff Phillips (jeffeusgs.gov) 9/19/2006. 


\section{USGS_SURFIT}

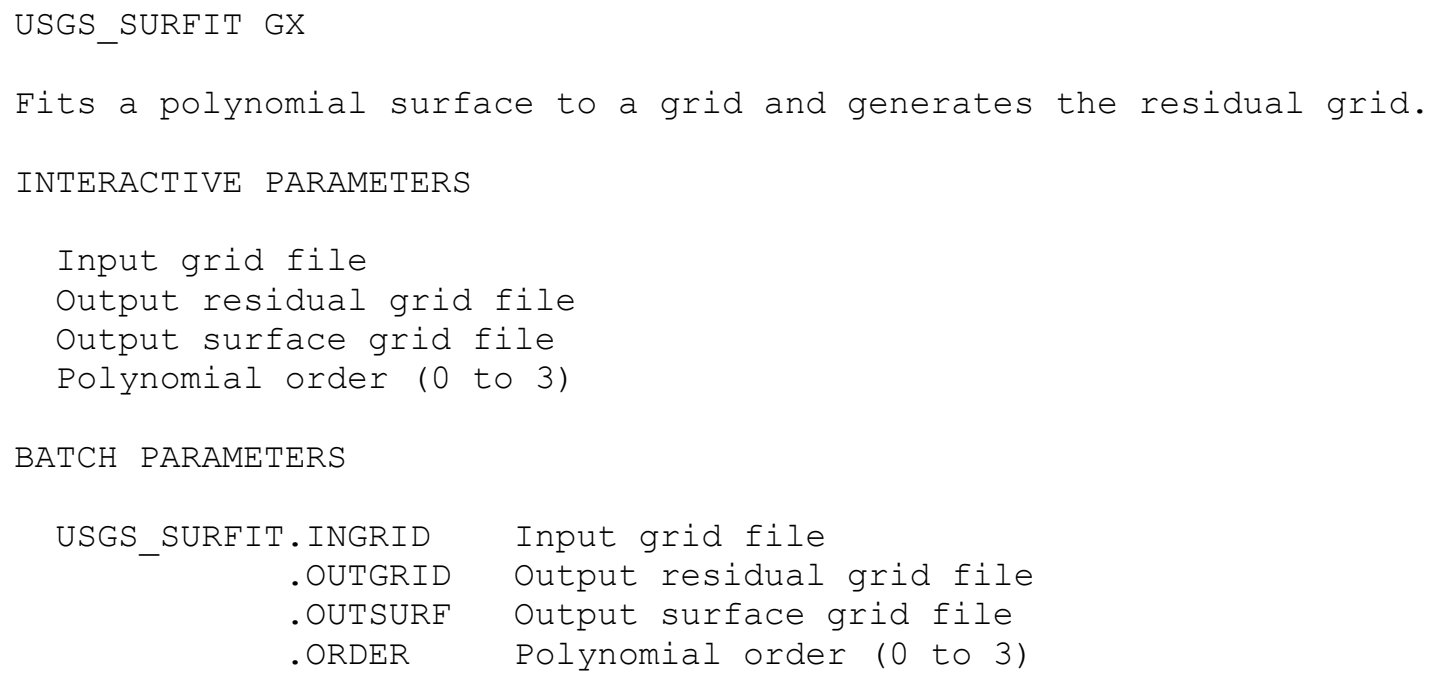

APPLICATION NOTES

Removes a polynomial surface of order 0 to 3 from an input grid. Creates both the residual grid and the polynomial surface grid. No data (DUMMY) areas are maintained in the output grids. The maximum number of columns is 8,000. Grid projection information is ignored.

Written by Jeff Phillips (jeff@usgs.gov) 2/21/2003. 


\section{USGS_T2VECT}

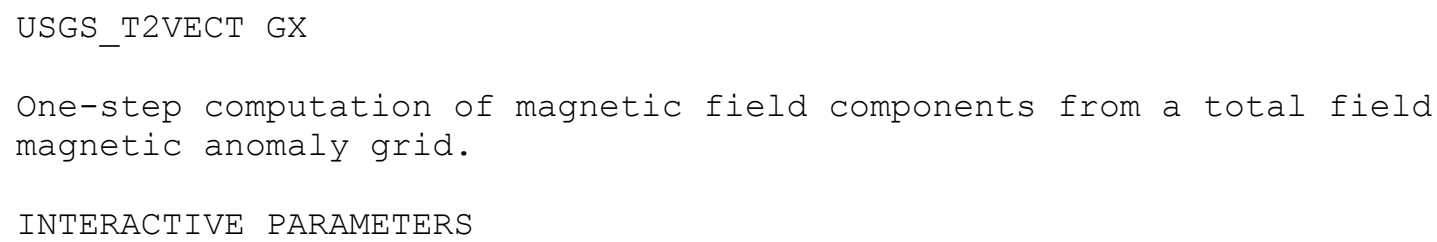

\section{APPLICATION NOTES}

This will not work well at low magnetic latitudes (within 20 degrees of the magnetic equator). The maximum number of columns in the expanded input grid is 16,384. Grid projection information is ignored.

Written by Jeff Phillips (jeff@usgs.gov) 06/05/2003. 
The maximum number of columns in the input grid is 8,000. Grid projection information is ignored.

\section{References:}

Nabighian, M.N., 1984, Toward a three-dimensional automatic interpretation of potential field data via generalized Hilbert transforms: Fundamental relations: Geophysics, v.49, no.6, p.780-786.

Thurston, J.B., and Brown, R.J., 1994, Automated source-edge location with a new variable pass-band horizontal-gradient operator: Geophysics, v.59, no.4, p.546-554.

Written by Jeff Phillips (jeff@usgs.gov) 07/17/2007. 


\section{USGS_TRANSPOS}

USGS_TRANSPOS GX

Transpose the rows and columns of a grid.

INTERACTIVE PARAMETERS

Input grid file

Output grid file

BATCH PARAMETERS

USGS_TRANSPOS.INGRID input grid

. OUTGRID output grid

APPLICATION NOTES

This is useful for row- or column-based processing such as filtering, extension, or depth analysis. The maximum number of columns in the input grid is 10,000 .

Written by Jeff Phillips (jeff@usgs.gov) 1/11/00. 


\section{USGS_TRIMGRD}

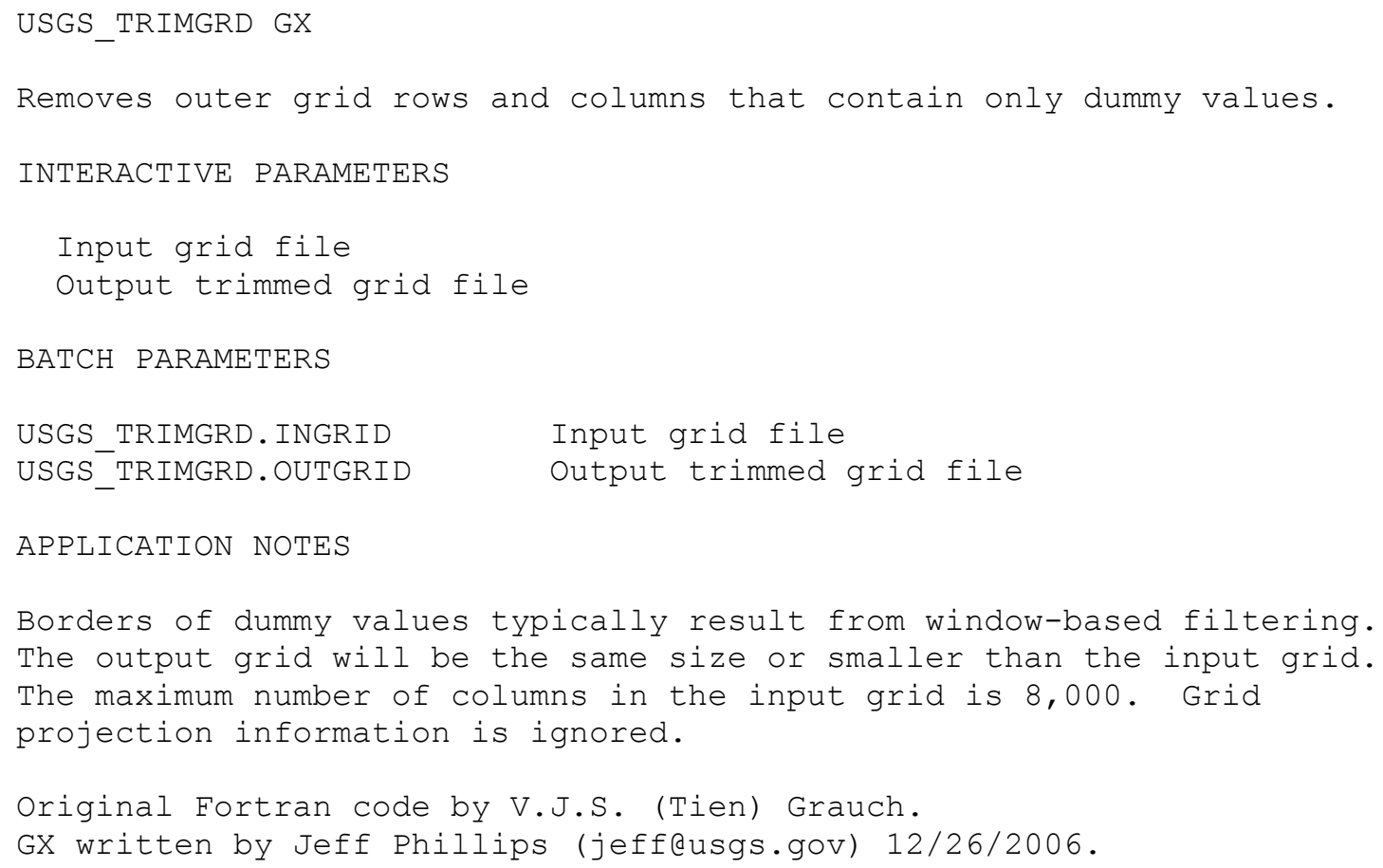




\section{USGS_UPCONT}

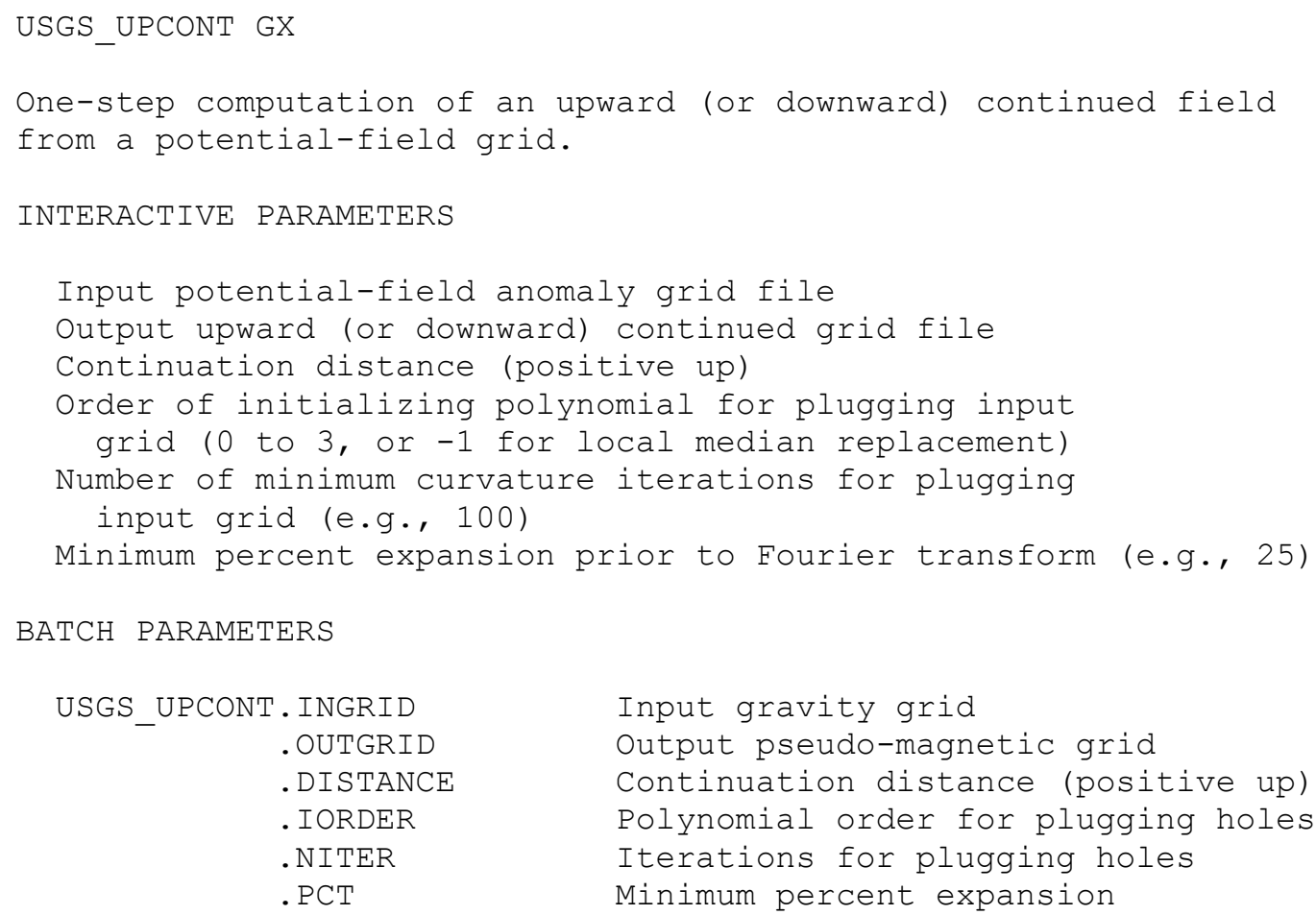

APPLICATION NOTES

Assumes the input data are on a flat, horizontal surface. Upward continuation is stable, but downward continuation may require additional low-pass filtering. The maximum number of columns in the expanded input grid is 16,384. Grid projection information is ignored.

Written by Jeff Phillips (jeffeusgs.gov) 06/10/2003. 


\section{USGS_VDFIL}

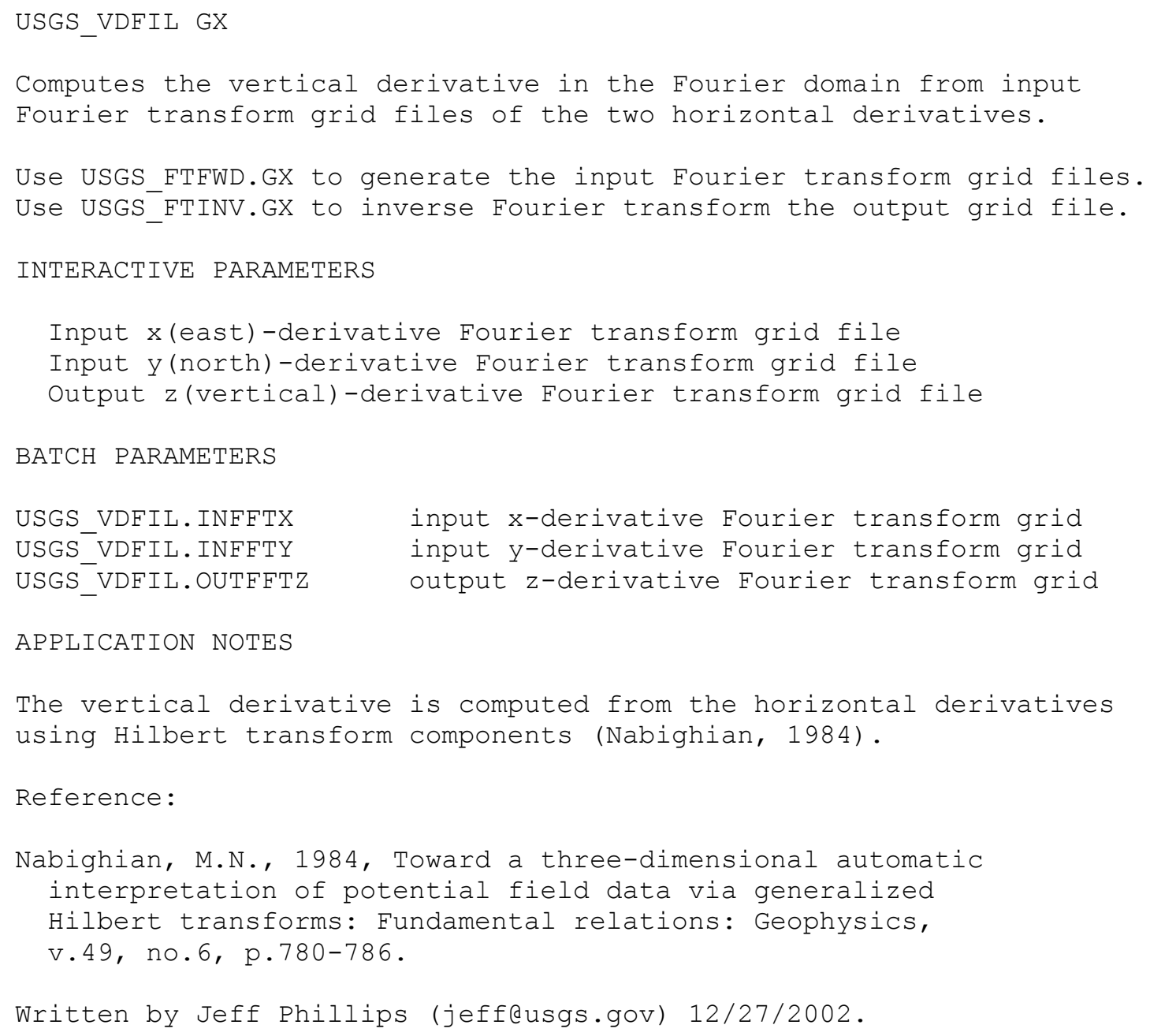




\section{USGS_VERTINT}

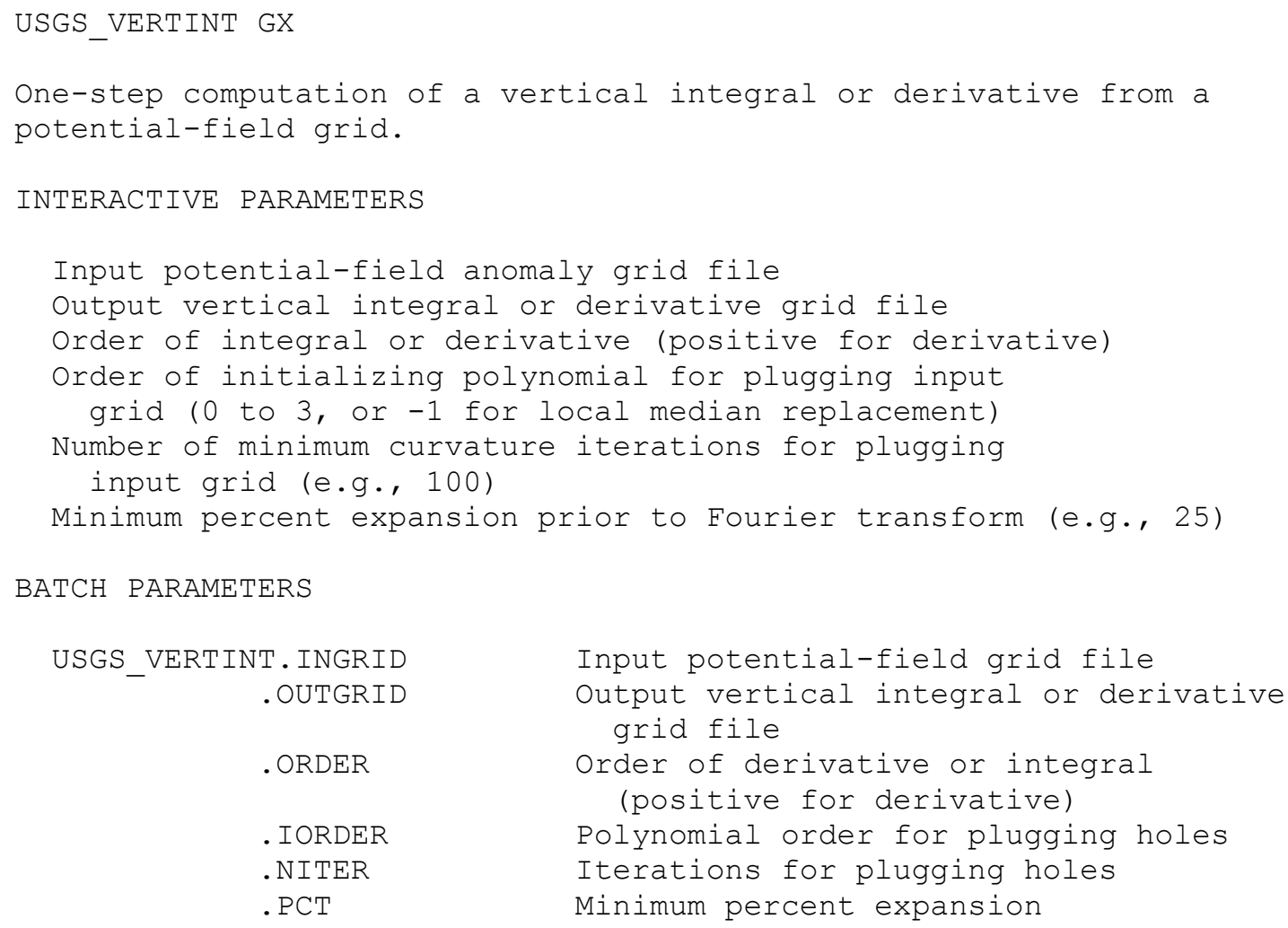




\section{Appendix 2: Converting FORTRAN code to a Geosoft GX using the GNU g77 and gcc compilers}

To convert Fortran code to a dynamic link library (dll) that can be called from a GX, Geosoft recommends using their version of the Fortran-to-C conversion program, f2c.exe, and the Microsoft Visual C++ compiler. This approach is described in the Geosoft GX Developer's Manual, available at http://www.geosoft.com, and was outlined in the version 1.0 GX release (Phillips and others, 2003).

An alternative approach for compiling dynamic link libraries from Fortran code for use with compiled GX's has been developed by Jim Roy and Tom Popowski of Northwest Geophysical Associates. This approach does not require the use of either f2c or Microsoft Visual C++. Instead, it uses the free, open-source compilers g77 and gcc. The object code placed in the dll is compiled directly from the Fortran source code along with the ancilliary $\mathrm{C}$ source code.

To use this alternate approach, the GX developer must install the free compilers and utilities of the open-source software package MinGW along with the command-line window system MSYS (described at http://www.mingw.org/ and available at http://sourceforge.net/). Also required are the free Geosoft GX Developer Toolkit (available at http://www.geosoft.com/downloads/), and either the free Geosoft Viewer (also available at http://www.geosoft.com/downloads/), or a licensed version of Geosoft Oasis montaj. In summary, the GX developer will need:

1. Either Geosoft Oasis montaj (licensed version) or the free Geosoft Viewer.

2. The free GX Developer software from Geosoft.

3. The free MinGW and MSYS software packages from sourceforge.net.

4. A text editor such as PFE32.EXE or Notepad. 


\section{INSTALLATION AND SETUP}

The Geosoft web site (http://www.geosoft.com) contains instructions for downloading and installing the current Geosoft Viewer (required if a licensed Oasis version is not installed) and the current GX Development software.

The MinGW and MSYS home page (http://www.mingw.org/) contains much useful information, but the current software versions are available only from sourceforge (http://sourceforge.net/). The installation files for the current versions are MinGW-5.1.3.exe and MSYS-1.0.10.exe. MinGW should be installed first, making sure $\mathbf{g 7 7}$ and $\mathbf{g + +}$ are checked and make is not checked. The installation will create a directory, C:MinGW, containing a minimalist GNU system. MSYS should be installed next, resulting in a directory, $\mathrm{C}:\lfloor$ msys $\backslash 1.0 \backslash$, containing the user file system.

A text editor will also be required. Two options are Windows Notepad or PFE, currently available from $h t t p: / / w w w . l a n c s . a c . u k / p e o p l e / s t e v e b / c p a a p / p f e / d e f a u l t . h t m$. Either can be accessed from a shortcut on the Windows desktop.

\section{GETTING STARTED}

GX development can be done under the /home/user_name directory of MSYS. You should be in this directory when you start MSYS by clicking on the blue "M" icon on your desktop. Create a new subdirectory called gxdev and move into it with the commands:

$$
\begin{aligned}
& \text { mkdir gxdev } \\
& \text { cd gxdev }
\end{aligned}
$$

This new directory will eventually contain:

1. Some library files, such as libGEODIST6.4.a and libGEOGX6.4.a.

2. A file called Makefile that we will use to create the libraries. 
3. The $\mathrm{C}$ source-code file wfuncs.c, containing the common source code for the wrapper functions called by the Fortran code to access the Geosoft library functions.

4. The $\mathrm{C}$ header file wrappers.h, containing the definitions for the wrapper functions. 5. Subdirectories for each GX to be developed.

\section{STEP 0: CREATING THE LIBRARIES AND FORTRAN WRAPPER FUNCTIONS}

The library files geogx.dll and geodist.dll, as supplied by Geosoft, cannot be used within the MinGW system. They must be converted to MinGW-compatible libraries. For GX Developer, version 6.4 , this is a matter of running make within the MSYS window on the makefile of $<$ Table A1>, which has the name Makefile. This makefile will need to be modified for later versions of GX Developer, hopefully only by changing the line assigning the OMVERS variable. Note that some long lines in $<$ Table A1 > have been wrapped.

I have chosen to use a single set of wrapper functions for all of my GX code. These wrapper functions are contained in the files wfuncs.c and wrappers.h, which reside in the gxdev directory. As new wrapper functions are needed, they can be added to these files. If old wrapper functions need to be modified, they should be thoroughly tested on all previously compiled GX code. If Geosoft makes changes to their basic library functions, as they did with the release of version 6.3, some wrapper functions may need to be rewritten. 


\section{CONVERTING AN EXAMPLE FORTRAN PROGRAM TO A GX}

We will be converting an example Fortran program (curv4.for) that reads a USGS binary grid file, operates on the grid file, and outputs a USGS binary post file. The resulting GX (usgs_curv4.gx) will read any grid file format supported by Geosoft, operate on the grid file, and output a USGS binary post file. It then calls another GX, either usgs_impost.gx for the free viewer or usgs_openpost.gx for the licensed version, to import the post file into a Geosoft database. The compiled GX files usgs_impost.gx and usgs_openpost.gx are included in the /gx directory on the ftp site. Copy them to the \Program Files\Geosoft \ Oasis montaj \gx directory or to the \Program Files\Geosoft \ Oasis montaj Viewerl gx directory on your computer. The compiled dynamic-link library files usgs_impost.dll and usgs_openpost.dll are included in the /binx directory on the ftp site. Copy them to the \Program Files $\backslash$ Geosoft $\backslash$ Oasis montaj $\backslash$ bin directory or to the $\backslash$ Program Files $\backslash$ Geosoft $\backslash$ Oasis montaj Viewer $\backslash$ bin directory on your computer. The source code files for usgs_curv4.gx are included in the /src/usgs_curv4 subdirectory on the ftp site.

Start by creating a working directory under your gxdev directory called usgs_curv4 with the command:

$$
\text { mkdir usgs_curv4 }
$$

This directory will contain the many files needed to create the GX. All GX's need:

1. A GX resource file that defines the user interface. This file has the suffix .grc and is compiled into a .gr file and a .grh file using the Geosoft grc compiler.

2. An rtf help file, created using WordPad, that is compiled along with the resource file.

3. A GX source code file with the suffix .gxc. This file is compiled, along with the output of the grc compiler, using the Geosoft gxc compiler to create the .gx file. 
In addition, GX's that use Fortran code need:

1. A Fortran source code file that has been modified to (a) remove all user interaction, (b) replace file I/O with calls to Geosoft wrapper functions, and (c) replace the main routine with a subroutine. This file has the suffix .f.

2. The $\mathrm{C}$ source code file for the wrapper functions. This file, which we call wfuncs.c, is kept in the gxdev directory.

3. The $\mathrm{C}$ header file for the wrapper functions. This file, which we call wrappers.h, is also kept in the gxdev directory.

4. A GX header file with suffix .gxh containing the prototype for the Fortran function called from the GX.

5. Another $\mathrm{C}$ source code file called $\mathbf{g x x} \_\mathbf{g} \mathbf{g} \_$name $\mathbf{x} . \mathbf{c}$ that contains the wrapper function called by the GX to access the Fortran code in the dynamic link library.

6. A file called Makefile for compiling the dynamic link library.

\section{STEP 1: EDITING THE FORTRAN SOURCE CODE}

The first file needed is the original Fortran source file curv4.for. Copy it from the Isrclusgs_curv4 subdirectory of the ftp site to your usgs_curv4 subdirectory. We will be editing this file to remove all user interaction, to replace all file I/O with calls to Fortran wrapper functions, and to convert it into a subroutine. The edited file will be called usgs_curv4.f, and it is also available on the ftp site. Here we examine the edited file a section at a time. The changes to the file are shown in uppercase bold text.

In this first section of code $(<$ Table A2 $>$ ), we have turned the program into a subroutine, using parameters in the call list to replace filenames and other parameters previously entered interactively by 
the user. The input character strings must be declared character*(*). Instead of testing for dvals, the USGS dummy value, we will be testing for the Geosoft DUMMY. The file unit numbers INF and IOF are initialized to zero. I have not found a successful way to pass an array to the subroutine, so the ITYPE array elements are passed as individual parameters.

In this second section of code (<Table A3>), the input grid file is opened using a wrapper function. The grid header is read using another wrapper function. If the grid contains too many columns, error message number 5007 in file usgs.err is displayed with the actual and maximum number of columns. The file usgs.err is contained on the ftp site in the /ger directory. It should be copied to either the \Program Files\Geosoft \Oasis montaj\ger subdirectory or the \Program Files\Geosoft Oasis montaj Viewerl ger subdirectory.

In this section (<Table A4>), the output post file is opened using a wrapper function. The first two rows of the input grid are read into the data array using another wrapper function.

Here (<Table A5>), a progress indicator is created and initialized using wrapper functions. The indicator is updated during each iteration of loop 2000 using another wrapper function. The next row of the input grid is read using a wrapper function.

Here (<Table A6>), the test for dvals is replaced by a test for the Geosoft DUMMY value. The output post record is written by a wrapper function. Not much else is changed in the data processing code.

In this final section (<Table A7>), another post record is written using a wrapper function. The progress indicator is updated to $100 \%$ at the end of all processing. If the input and output files exist, they are closed using wrapper functions. We are done with the Fortran code. 


\section{STEP 2: CREATING THE USER INTERFACE}

The next step is to design the user interface for the GX. This is done in a resource file called usgs_curv4.grc, displayed in $<$ Table A8 $>$ and available on the ftp site. The interface uses two filename entry boxes for the input grid file and the output post file, and five list entries for the options. There are three buttons - OK, which returns 0, Cancel, which returns -1, and Help, which calls the help resource text file usgs_curv4.rtf.

Another common interface element not seen in this example is the text entry box. Here is an example:

$$
\text { EDIT, , ,10, "Minimum amp1itude", , REAL, -1.e30 }
$$

Before we forget, let's create the help file, usgs_curv4.rtf (<Table A9>). This is just a text file that has been converted to RTF format using Wordpad. You can copy it from the ftp site.

\section{STEP 3: CREATING THE GX SOURCE CODE}

Next we will create the GX source code file, usgs_curv4.gxc (<Table A10>). Like all the source code, this can be found on the ftp site.

The example text entry box, which was not used in the resource file, has been carried through in the gxc code using lines that have been commented out. The GX should create and open a new database, so there is no need for a closing message. For more information on .grc and .gxc file formats, see the GX Developer Users Guide and Reference Manual available from the Geosoft website. Details

on the use of Geosoft library functions, such as DisplayMessage_SYS, can be found in C:IProgram Files\GeosoftlGX Developer|gxlincludel*.gxh.

The GX source code file requires a GX header file called usgs_curv4.gxh (<Table A11 $>$ ). 


\section{STEP 4: COMPILING THE GX}

At this point, we can compile the GX resource and help files using:

$$
\text { grc usgs_curv4 }
$$

Then we can compile the GX using:

$$
\text { gxc usgs_curv4 }
$$

This assumes that the PATH environment variable contains the location of the grc and gxc compilers, C:IProgram Files\GeosoftlGX Developer|gxlbin. The output of the gxc compiler is the file usgs_curv4.gx. This can be copied to the Oasis montaj gx directory using Windows drag-and-drop or by using the MSYS command

cp usgs_curv4.gx /c/Program \Files/Geosoft/Oasis \montaj\Viewer/gx

for the viewer, or

cp usgs_curv4.gx /c/Program \Files/Geosoft/Oasis \montaj/gx

for the licensed version.

\section{STEP 5: CREATING THE DYNAMIC LINK LIBRARY}

To create the dynamic link library (DLL), you will need the libraries, libGEOGX6.4.a and libGEODIST6.4.a, which are located in the gxdev directory and which were created in Step 0 above. You will need the edited Fortran source code file, usgs_curv4.f, located in the gxdev/usgs_curv4 subdirectory and created in Step 1 above, and the wrapper function source code file wfuncs.c, located in the gxdev directory. You will need to edit the wrapper function header file, wrappers.h in the gxdev directory to include the definition for the new curv4 wrapper function just before the final \#endif 
$(<$ Table A12>). You will also need one more source code file called gxx_curv4.c in the gxdev/usgs_curv4 subdirectory (<Table A13>).

The final file needed is a makefile, called Makefile, which resides in the gxdev/usgs_curv4 subdirectory (<Table A14>). This makefile is set up to compile the DLL and copy it to the bin directory of the Geosoft viewer. You can change the final destination to the bin directory of the licensed version by using the line that is commented out. Run the makefile using the command make in the MSYS window while in the gxdev/usgs_curv4 subdirectory.

\section{STEP 6: TESTING AND DEBUGGING}

Fire up Oasis montaj or the Oasis montaj Viewer and test the GX. You will need to authorize the GX the first time you run it. At this point, several things can happen:

1. The GX can run and produce the desired output. Congratulations, you have created a successful GX!

2. The GX can exit without producing output or messages.

3. The GX can produce an error message.

4. The GX can crash Oasis.

Debugging a GX is difficult. There are often many source code files involved, and an error in any one of them, even as insignificant as a missing semicolon, can lead to failure. Problems involving syntax errors can be avoided by referring to the many successful examples on the ftp site. Problems involving logic errors in the Fortran code can be tracked down through a combination of:

1. Placing go to 9999 statements in various places in the Fortran code, until you can isolate the loop or subroutine generating the problem. 
2. Using error wrapper functions to print out the values of suspect variables. For example, error number 6001 in the usgs.err file will print the values of up to five variables, using code such as ca11 RegisterErr_WF(6001, 'usgs')

ca11 SetErrParmR_WF(1, radius)

ca11 SetErrParmI_WF(2,indx) 


\section{Table A1. Listing of Makefile for creating the Geosoft libraries.}

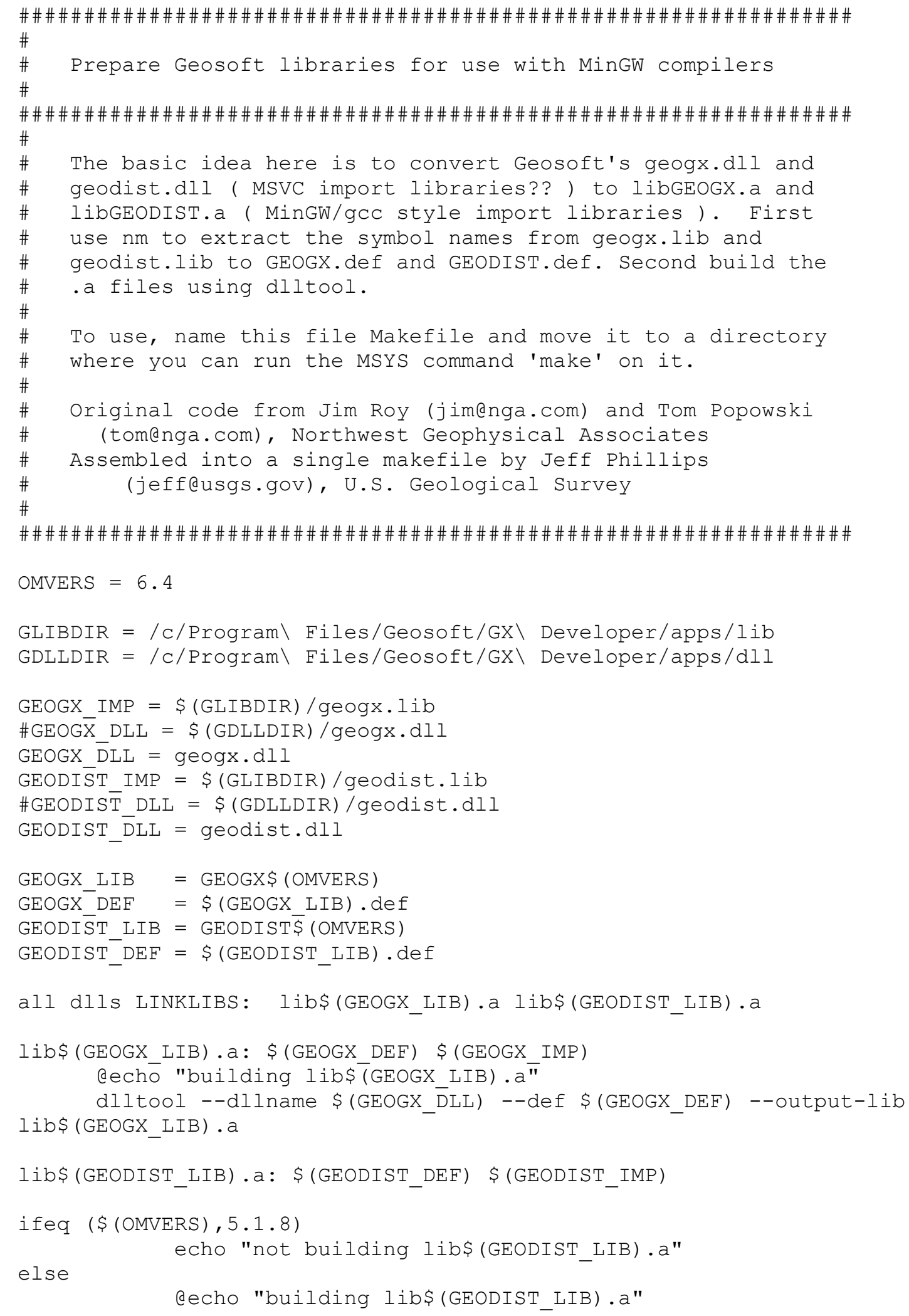




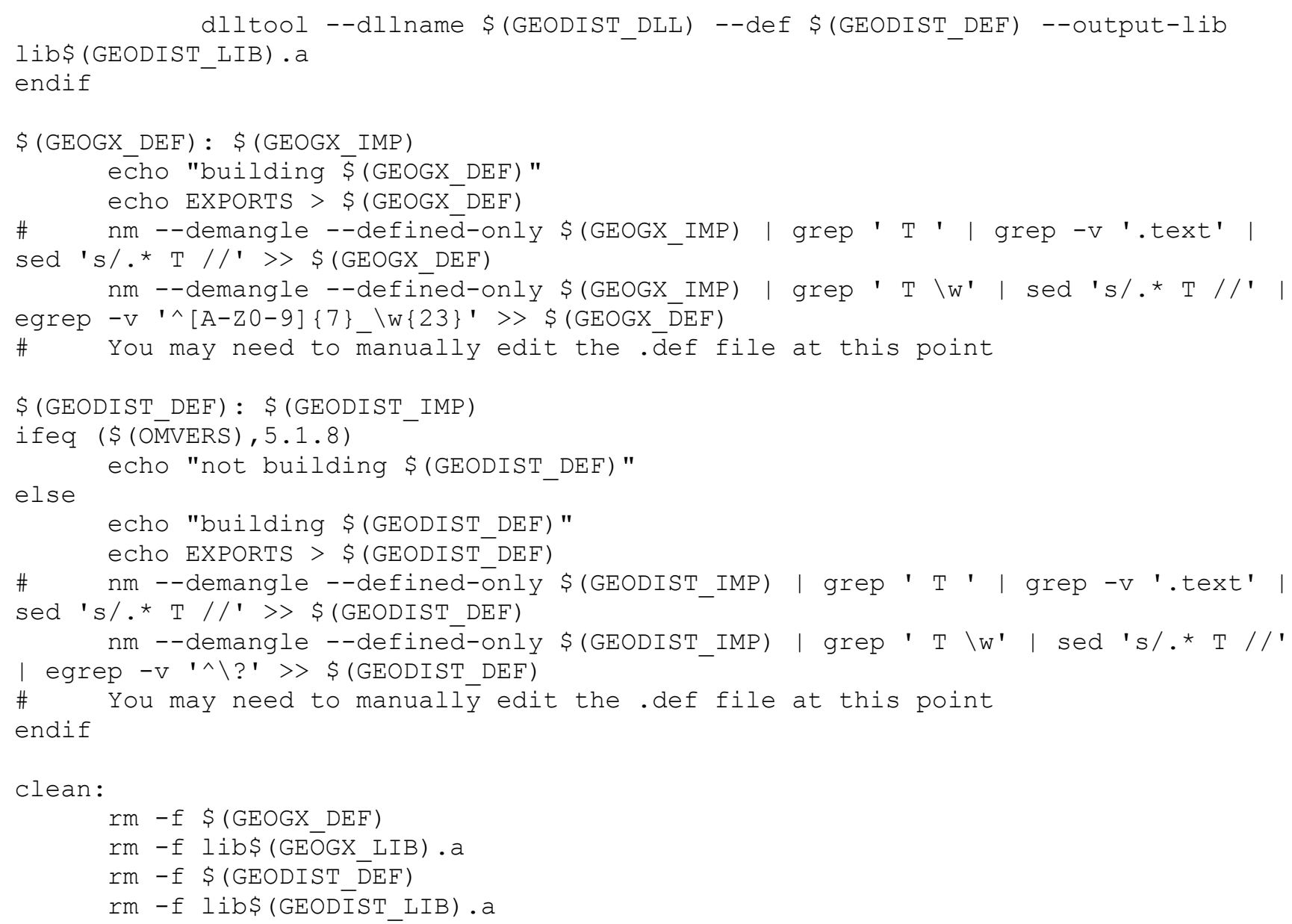


Table A2. Part 1 of the edited Fortran source code file, usgs_curv4.f.

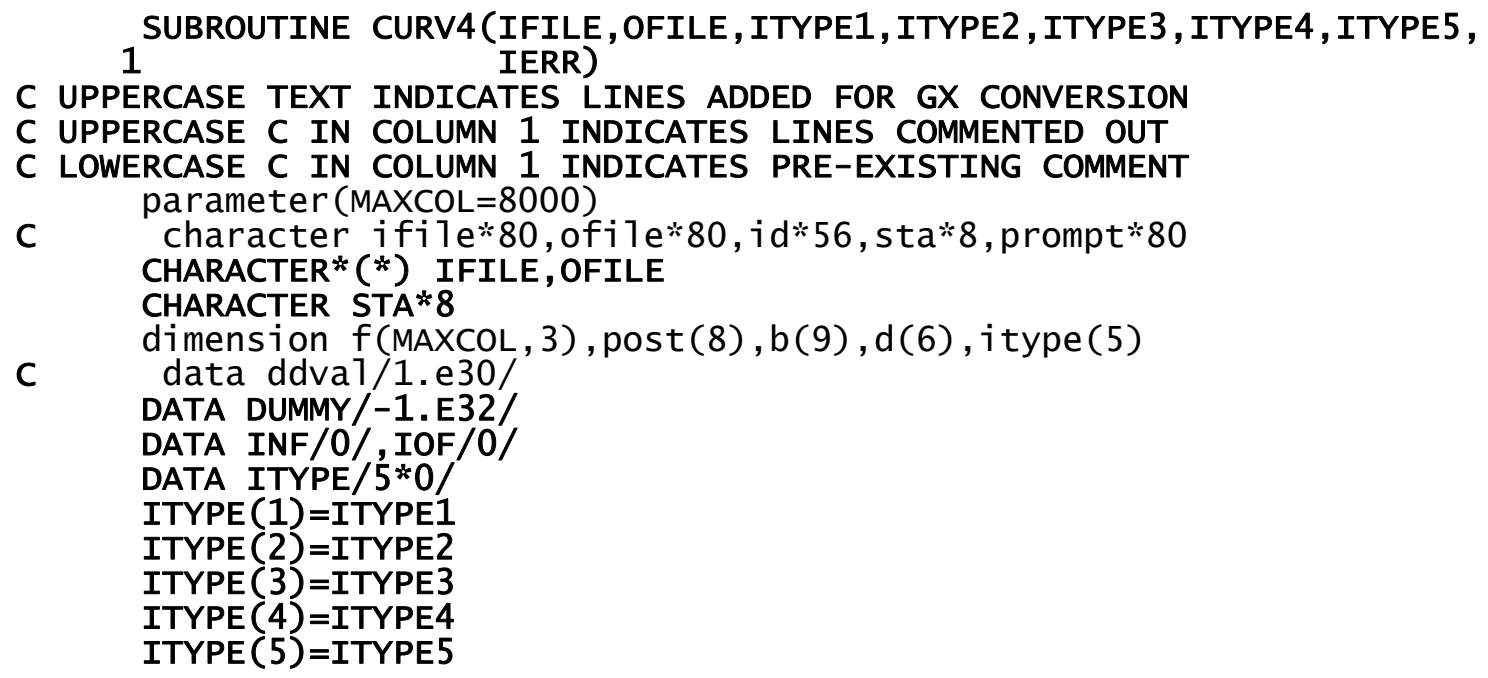


Table A3. Part 2 of the edited Fortran source code file.

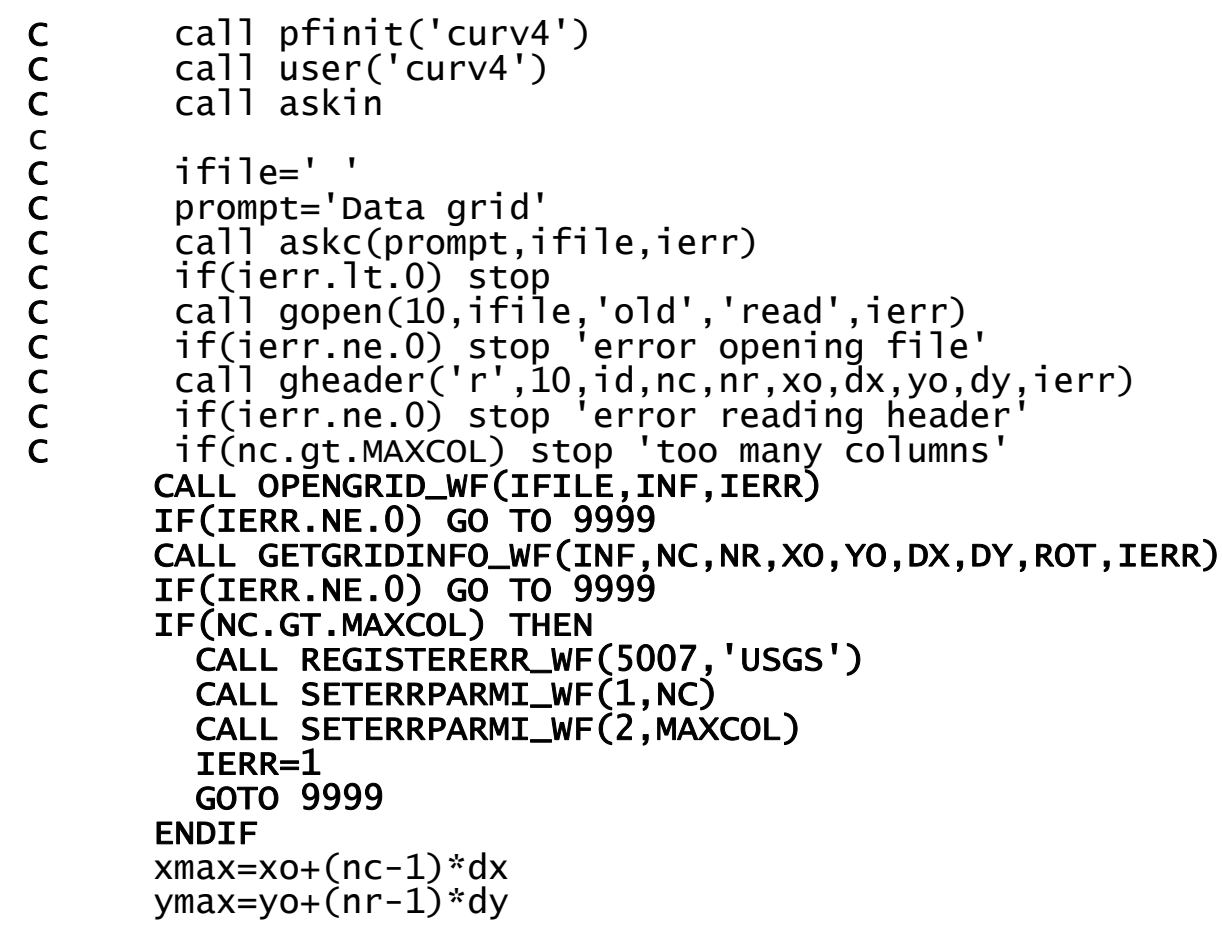


Table A4. Part 3 of the edited Fortran source code file.

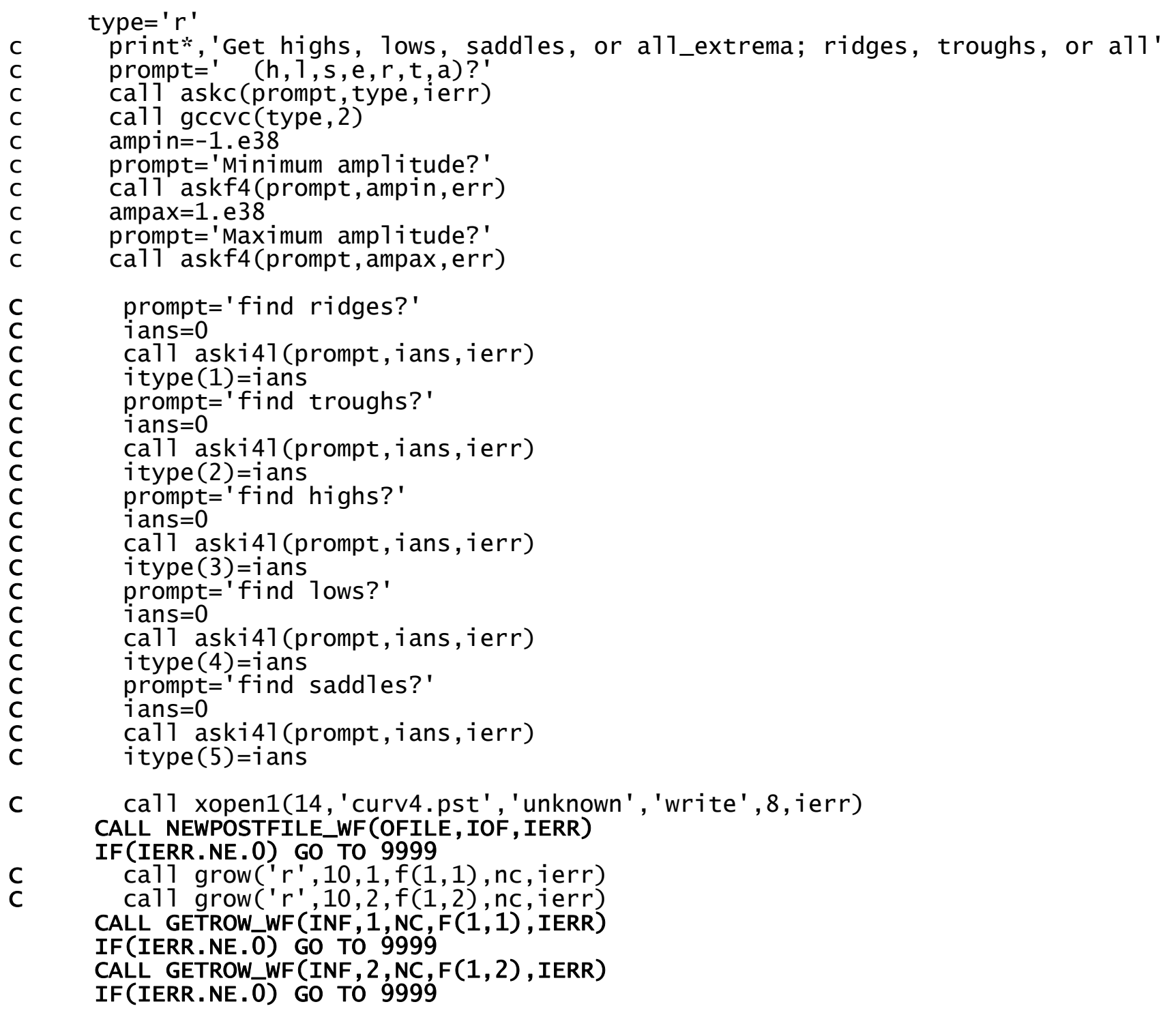


Table A5. Part 4 of the edited Fortran source code file.

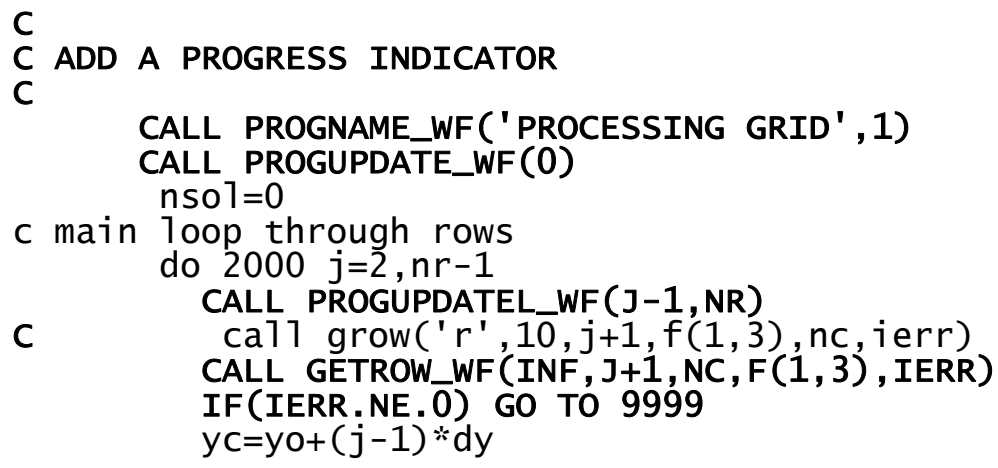


Table A6. Part 5 of the edited Fortran source code file.

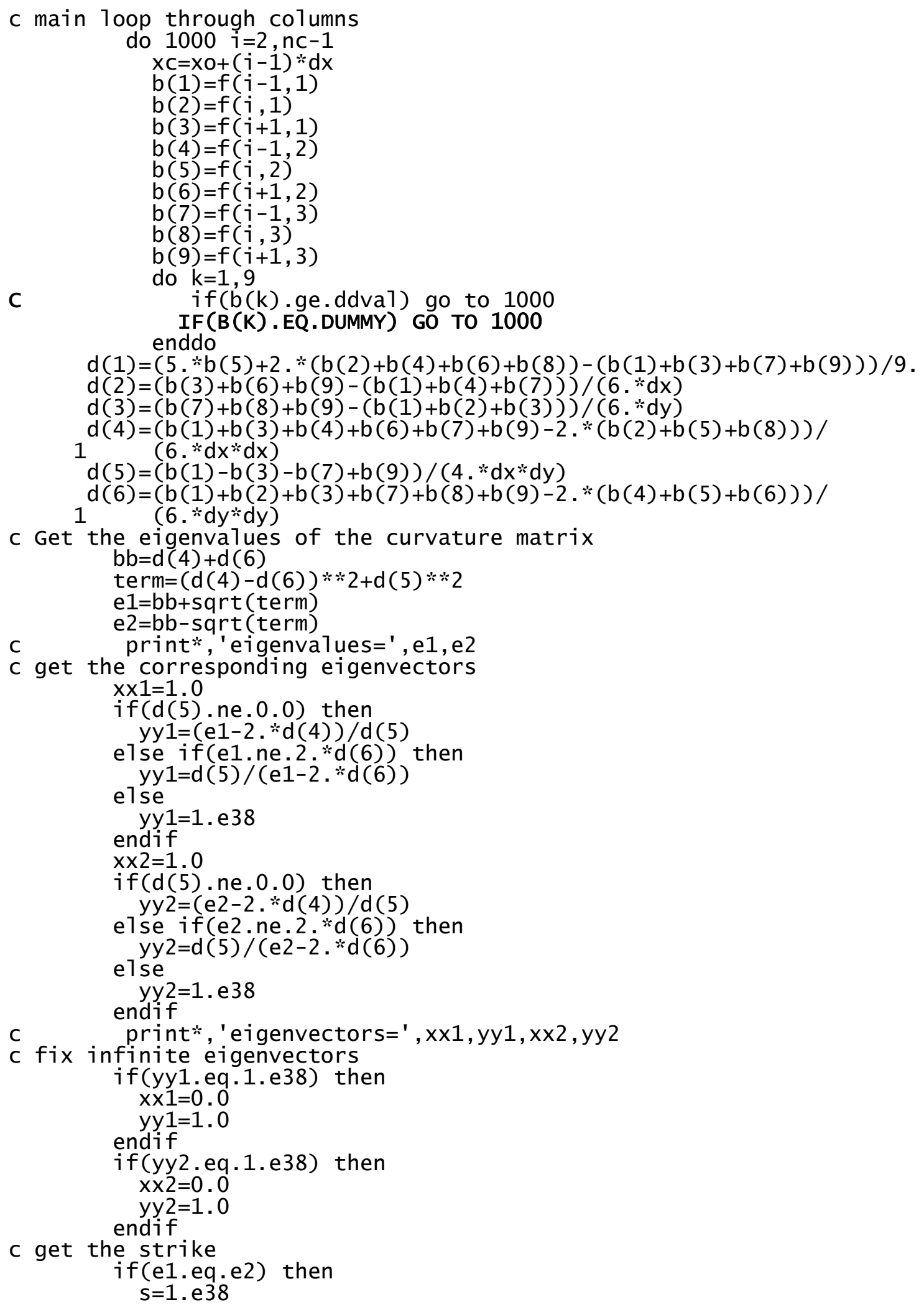




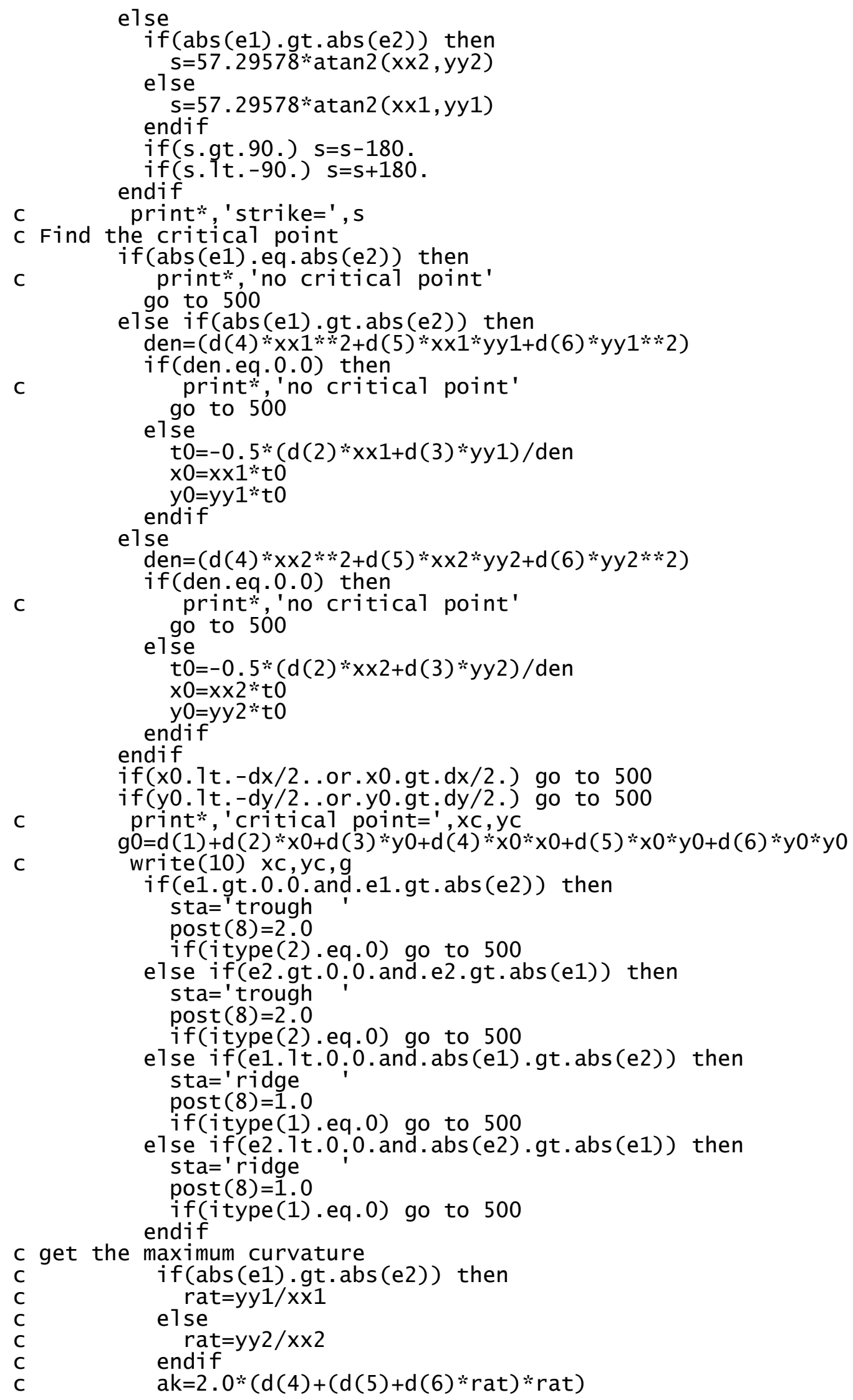

C 


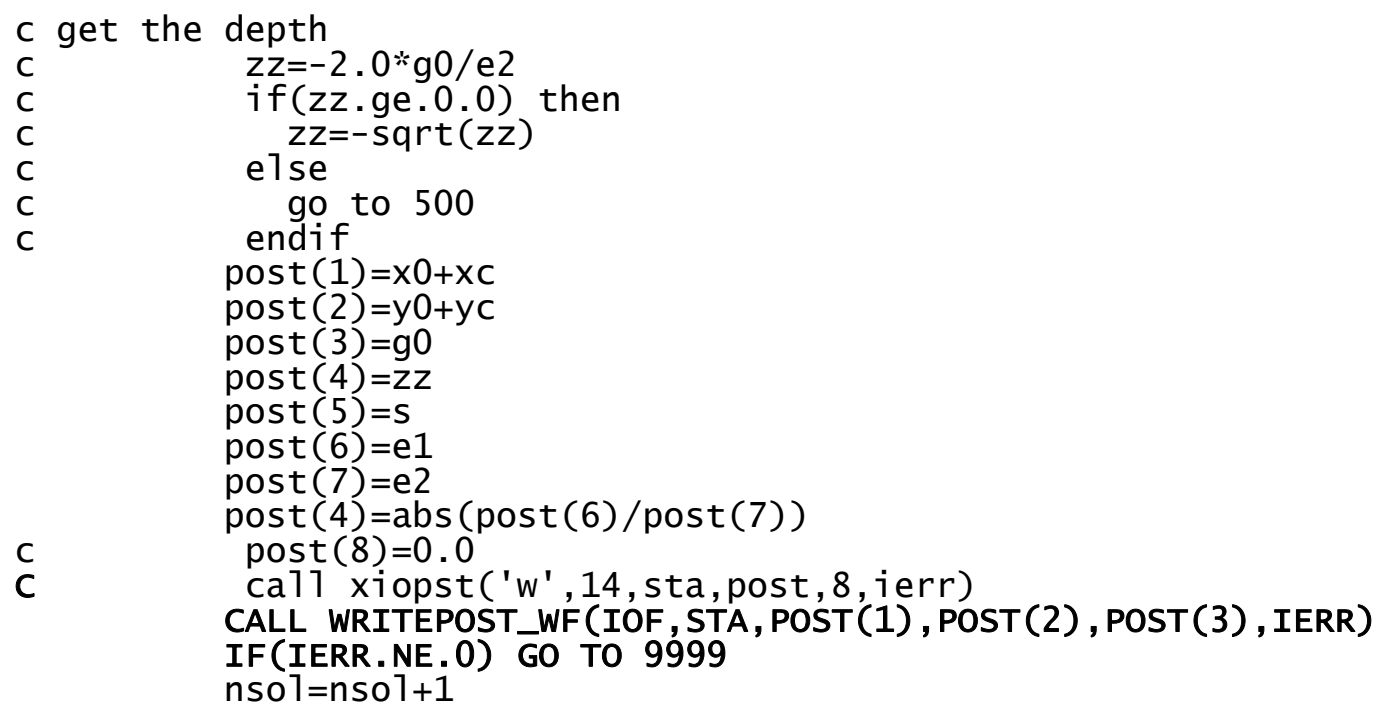


Table A7. Part 6 of the edited Fortran source code file.

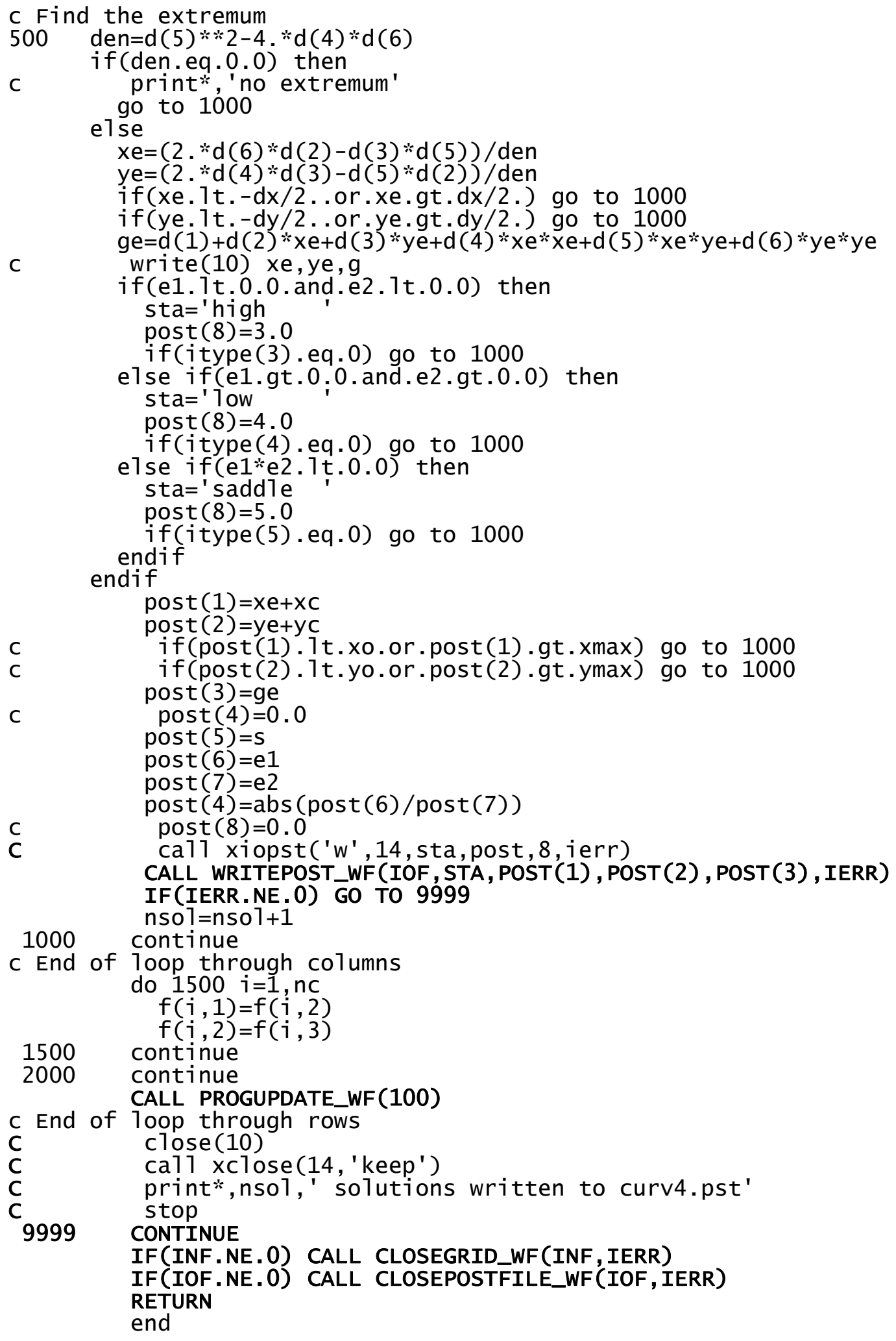


Table A8. The GX resource source code file, usgs_curv4.grc.

// USGS_CURV4.GRC

//----------

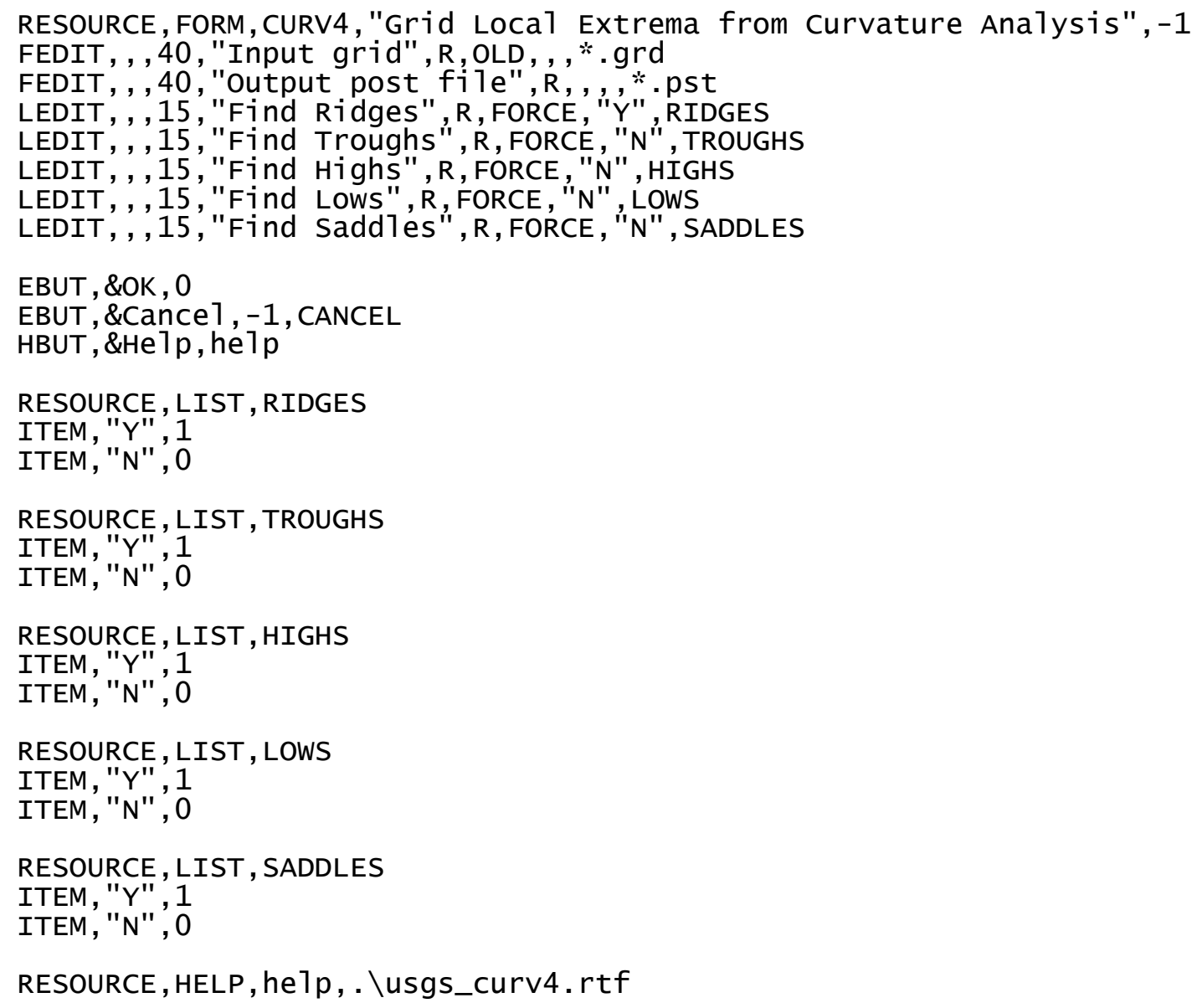


Table A9. The GX help file, usgs_curv4.rtf.

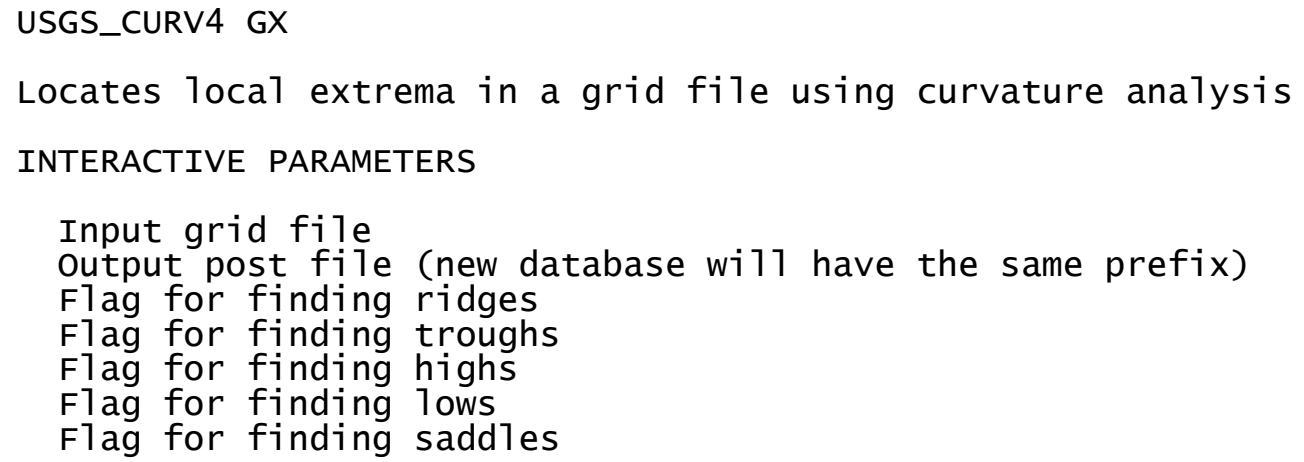

USGS_CURV4.INGRID

USGS_CURV4. OUTPOST

USGS_CURV4.RIDGES

USGS_CURV4.TROUGHS

USGS_CURV4. HIGHS

USGS_CURV4. LOWS

USGS_CURV4.SADDLES

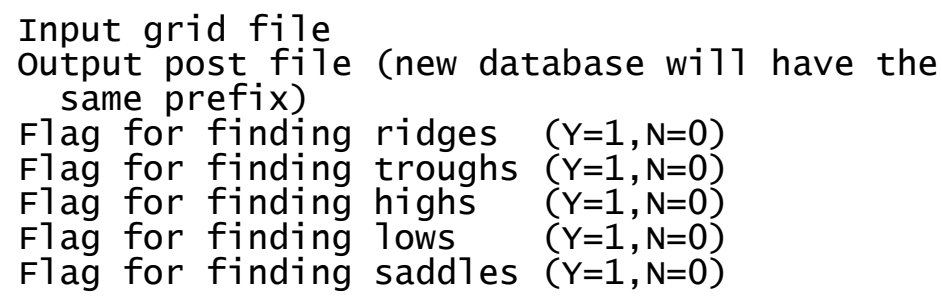

APPLICATION NOTES

The output post file and database have the following channels:

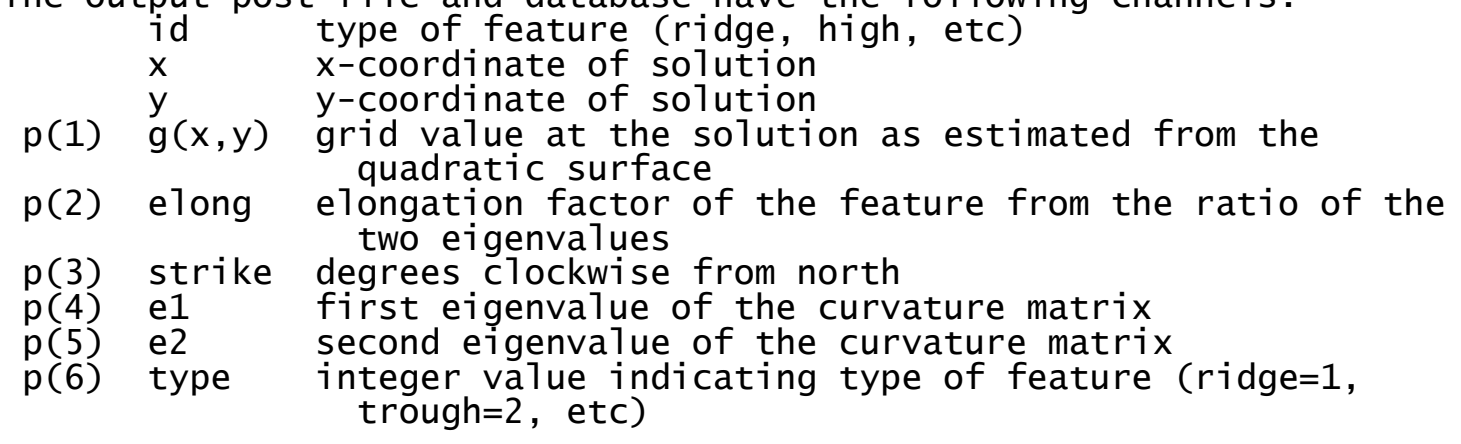

Reference:

Phillips, J.D., Hansen, R.O., and Blakely, R.J., 2007, The use of curvature in potential-field interpretation: Exploration Geophysics, v. 38, p.111-119.

Written by Jeff Phi11ips (jeff@usgs.gov) 9/14/2006. 
Table A10. The GX source code file, usgs_curv4.gxc.

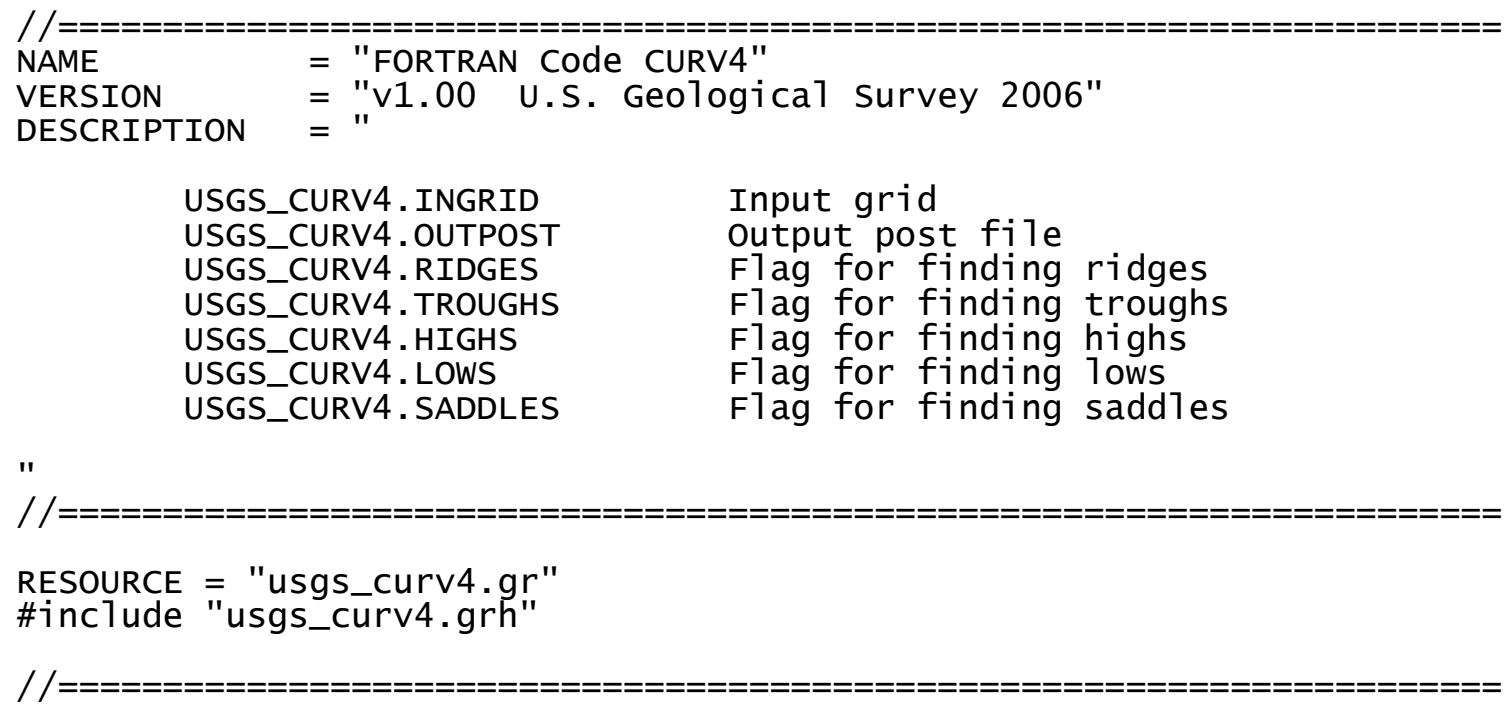

\#include <a11.gxh>

\#include "usgs_curv4.gxh"

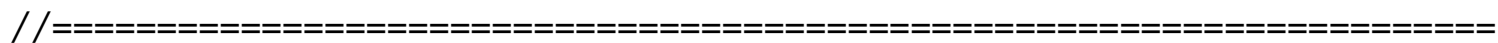

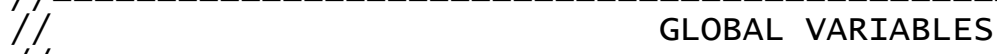

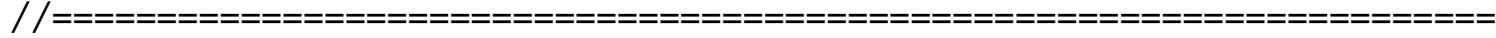

DGW Diag; // Dialogue handle

string(GS_MAX_PATH) sInGrid, soutPost, sOutGDB;

int (5) iтype;

//real rampin;

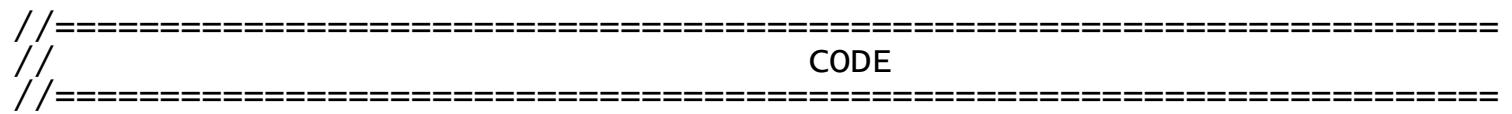

\{

if (iInteractive_sys()) \{

// --- Create the Dialogue ---

Diag = create_DGW("CURV4");

// --- Set any Defaults from INI ---

SetInfoSYS_DGW(Diag,_CURV4_0, DGW_FILEPATH, "USGS_CURV4", "'INGRID"); SetInfoSYS_DGW (Diag,_CURV4_1, DGW_FILEPATH, "USGS_CURV4", "OUTPOST") ;

SetInfoSYS_DGW (Diag, _CURV4_2, DGW_LISTALIAS, "USGS_CURV4" , "RIDGES");

SetInfoSYS_DGW (Diag,_CURV4_3, DGW_LISTALIAS, "USGS_CURV4", "TROUGHS") ;

SetInfoSYS_DGW (Dịag,_CURV4_4, DGW_LISTALIAS, "USGS_CURV4", "HIGHS") ;

SetInfoSYS_DGW (Diag,_CURV4_5, DGW_LISTALIAS, "USGS_CURV4", "LOWS");

SetInfoSYS_DGW(Diag,_CURV4_6,DGW_LISTALIAS, "USGS_CURV4", "SADDLES") ;

// SetInfosYS_DGW(Diag,_CURV4_7, DGW_TEXT, "USGS_CURV4", "AMPIN");

// --- Run the Dialogue ---

if (iRunDialogue_DGW(Diag) !=0) Cance1_SYS(); 


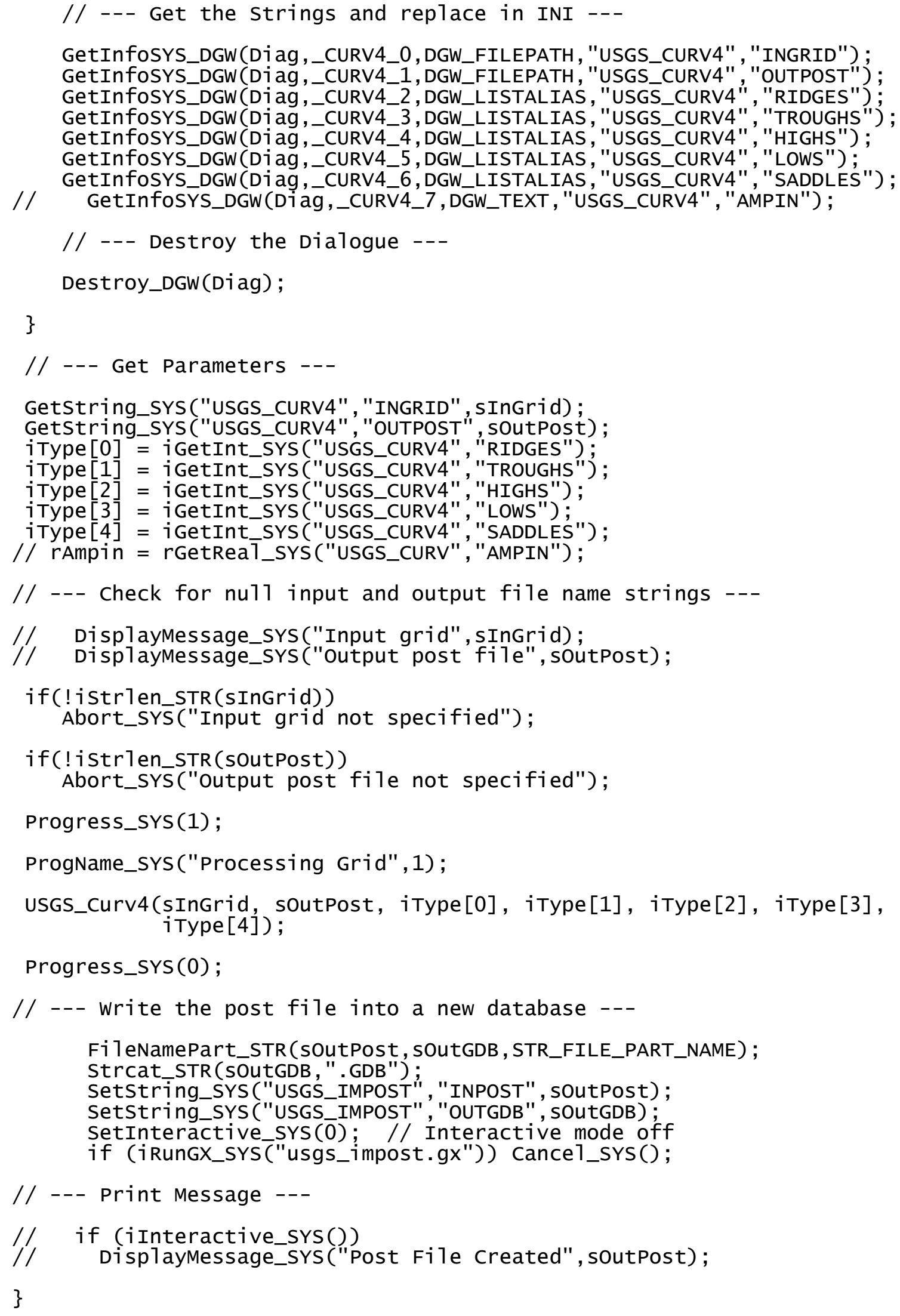


Table A11. The GX header file, usgs_curv4.gxh.

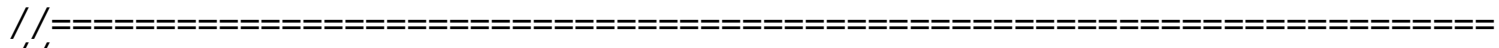

$/ /$

// usgs_curv4.gxh - Prototype for wrappers for CURV4 Fortran subroutine $1 /$

$/ / 1$

\# jifndef CURV4_GXH_DEFINED

\#define CURV4_GXH_DEFINED

\#define USGS_Curv4(A, B, C,D,E, F, G) IUSGS_Curv4(A, sizeof(A), B, sizeof(B) , C, D, E , F , G)

[usgs_curv4] void

IUSGS_Curv4(string, // input grid file

int, // string length

string, // output post file

int, // string length

int, // itype1

int, // itype2

int, // itype3

int, // itype4

//

int); $/ /$ itype 5

int(5)); / itype

\#endif 
Table A12. Code fragment added to the end of the wrapper functions header file, wrappers.h.

// USGS_Curv4.f

int curv4_(char *pcInfile, char *pcoutfile, long *itype1, long *itype2, long *itype 3 , long *itype 4 , long *itype 5 , long *ierr,

long infile_len, long outfile_len); 
Table A13. The C source code file, gxx_curv4.c.

/ //static char *_THIS_FILE_NAME_ = "\$Id://depot/src/usgs/gxx_usgs.C\#4 $\$ "$;

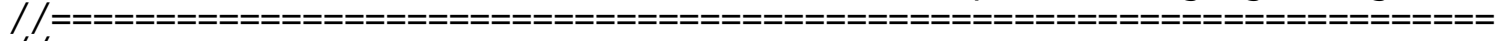

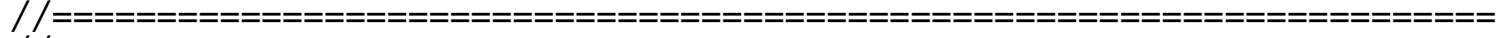

$/ / /$ gxx_curv4.c

// USGS FORTRAN method wrappers.

$/ /$

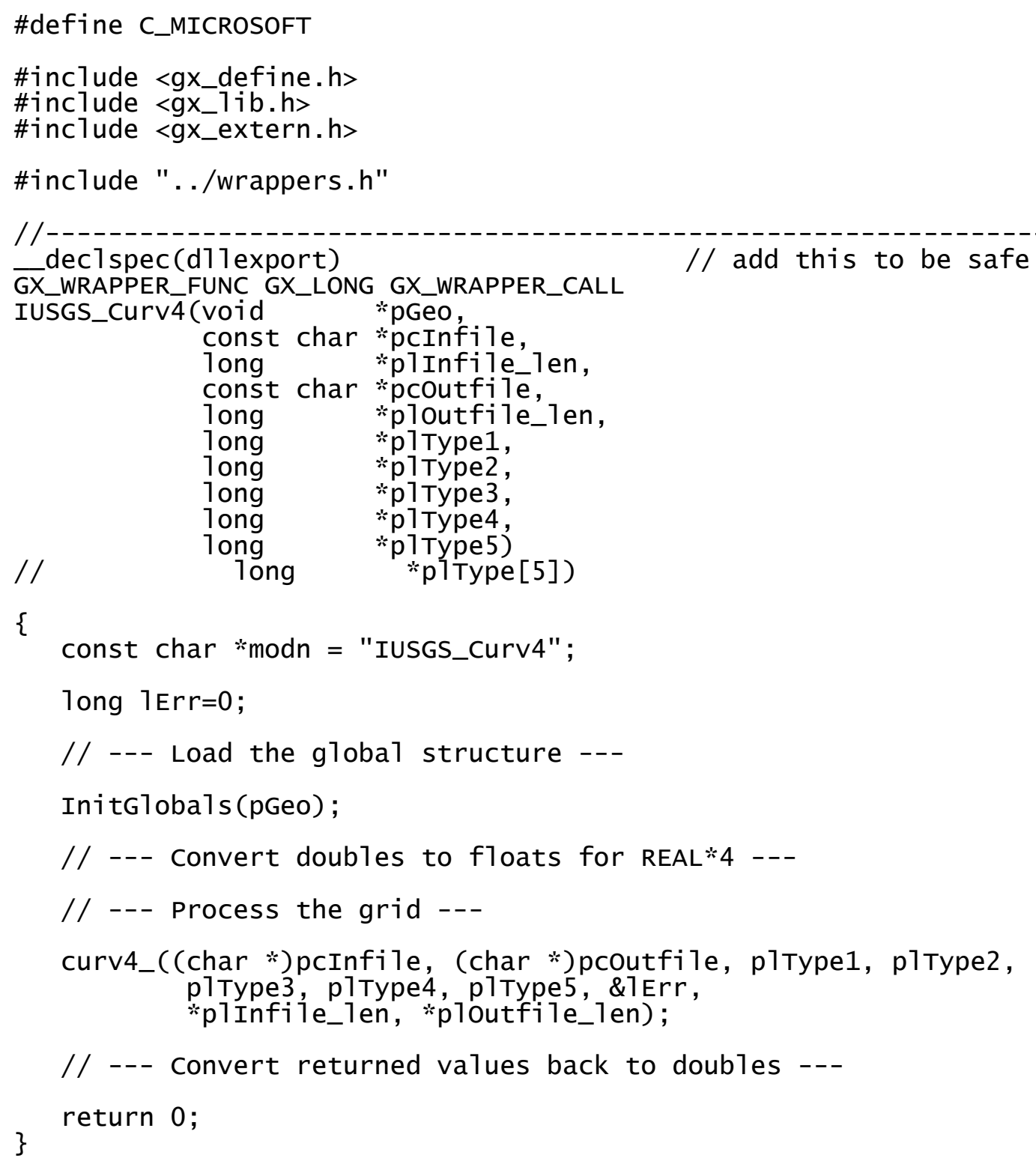


Table A14. The makefile for creating the GX dynamic-link library, Makefile.

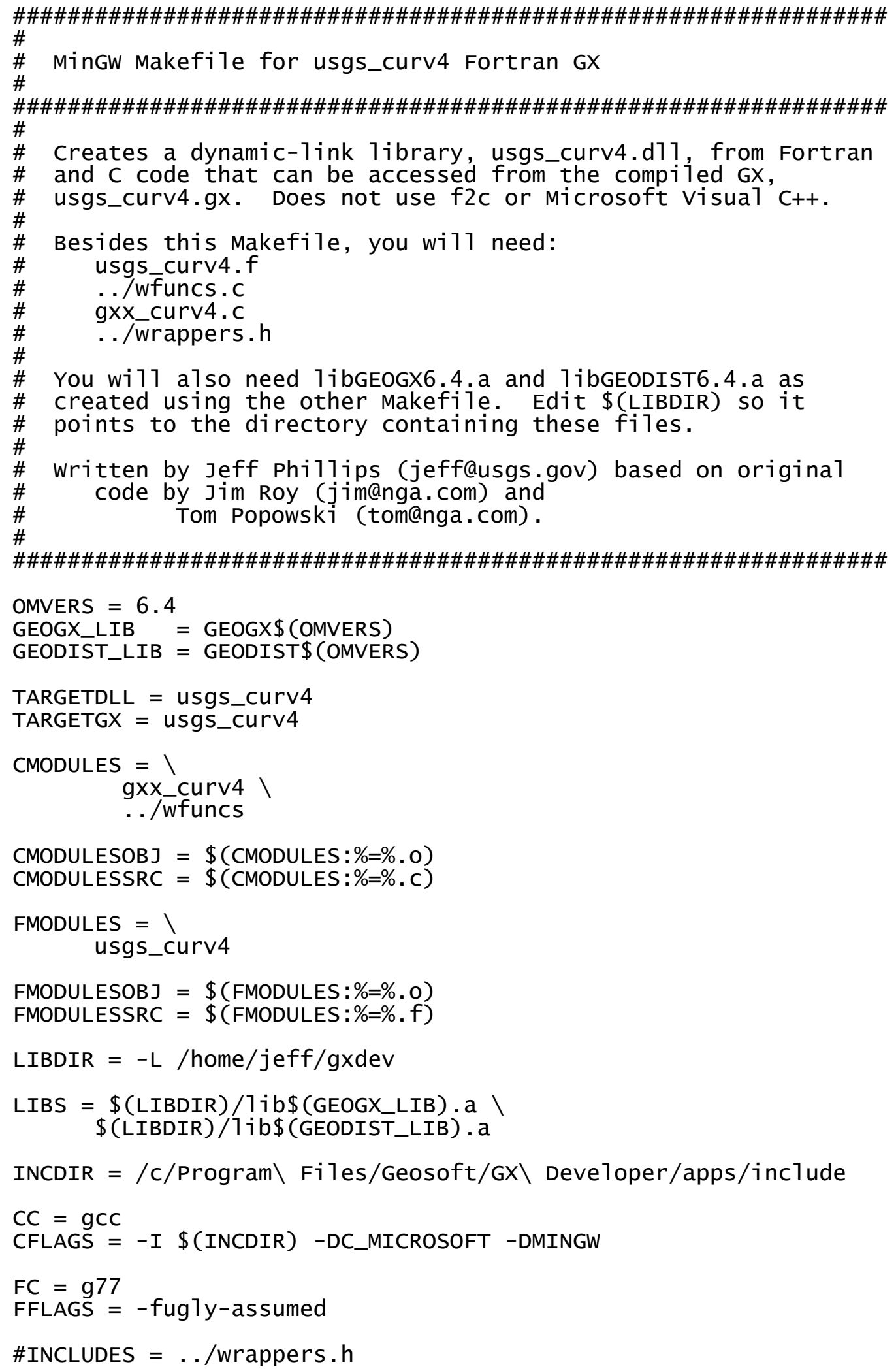


\$(TARGETDLL): \$(FMODULESOBJ) \$(CMODULESOBJ)

d11 tool --export-a11-symbols -z \$(TARGETDLL). def $\$ \wedge$

exports.o $\$ \wedge$

d11too 1 -d \$(TARGETDLL). def - 1 \$(TARGETDLL).a -D \$(TARGETDLL).d11 -e

g77 - shared -o \$(TARGETDLL).d11 \$(LIBDIR) \$^ exports.o -1GEOGX\$(OMVERS)

-1GEODIST\$(OMVERS)

CP \$(TARGETDLL).d71/c/Program\Files/Geosoft/Oasis \montaj\Viewer/bin

cp \$(TARGETDLL).d11/c/Program\Files/Geosoft/Oasis \montaj/bin

cp \$(TARGETGX).gx /C/Program \Files/Geosoft/Oasis\montaj\Viewer/gx

cp \$(TARGETGX).gX /c/Program \Files/Geosoft/Oasis \montaj/gx

$\$(C M O D U L E S O B J): \quad \$(C M O D U L E S S R C)$

$\$(C C)-C \$ * . C \quad \$(C F L A G S)-0 \$ * .0$

\$(FMODULESOBJ): \$(FMODULESSRC)

$\$(F C)-C \$ * . f \quad \$(F F L A G S)-0 \$ * .0$

clean:

$r m-f$ core $\$($ TARGETDLL $) * . o * . a * . d e f$

.c.o: Makefile

$\$(C C) \$(C F L A G S)-c \quad \$<-0 \$ @$

.f.o: Makefile

$\$(F C) \quad \$(F F L A G S)-c \quad \$<-0 \$ @$ 